Portland State University

PDXScholar

$5-5-1995$

\title{
Testability Design and Testability Analysis of a Cube Calculus Machine
}

Lixin Zhou

Portland State University

Follow this and additional works at: https://pdxscholar.library.pdx.edu/open_access_etds

Part of the Electrical and Computer Engineering Commons Let us know how access to this document benefits you.

Recommended Citation

Zhou, Lixin, "Testability Design and Testability Analysis of a Cube Calculus Machine" (1995). Dissertations and Theses. Paper 4911.

https://doi.org/10.15760/etd.6787

This Thesis is brought to you for free and open access. It has been accepted for inclusion in Dissertations and Theses by an authorized administrator of PDXScholar. Please contact us if we can make this document more accessible: pdxscholar@pdx.edu. 


\section{THESIS APPROVAL}

The abstract and thesis of Lixin Zhou for the Master of Science in Electrical and Computer Engineering were presented May 5, 1995, and accepted by the thesis committee and the department.

COMMITTEE APPROVALS:

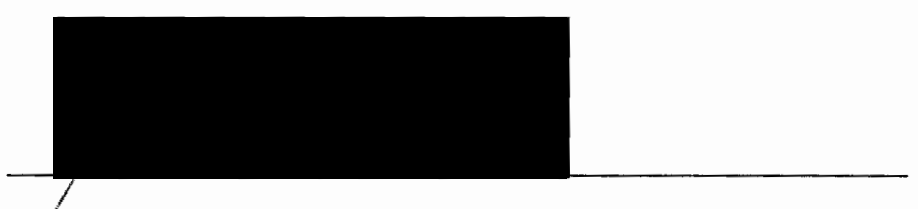

Dr. Marek Perkowski, Chair

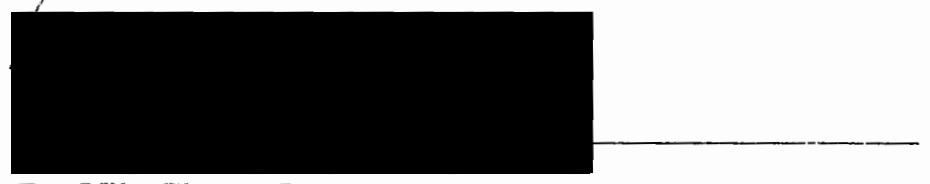

Dr. Yih-Chyun Jenq

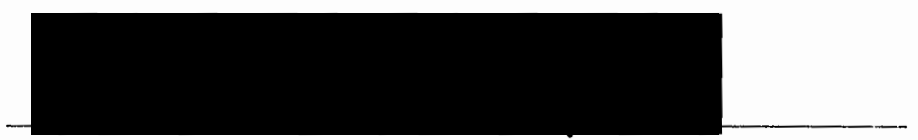

Dr. Maria Balogh

Representative of the Office of Graduate Studies

DEPARTMENT APPROVAL:

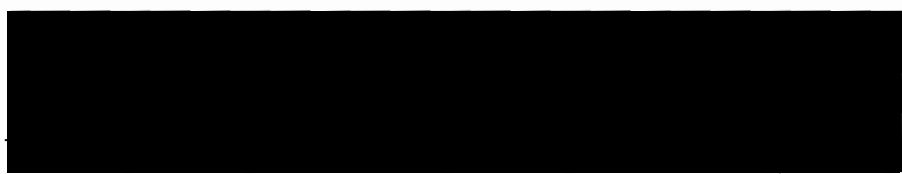

Dr. Rolf Schaumann, Chair

Department of Electrical Engineering

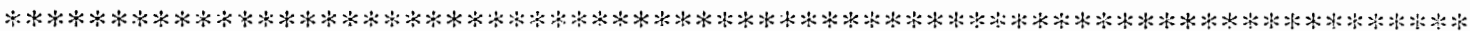

ACCEPTED FOR PORTLAND STATE UNIVERSITY BY THE LIBRARY

by

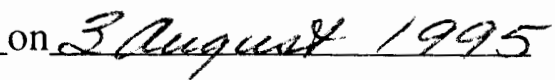




\begin{abstract}
An abstract of the thesis of Lixin Zhou for the Master of Science in Electrical and Computer Engineering presented May 5, 1995.
\end{abstract}

Title: Testability Design and Testability Analysis of a Cube Calculus Machine

Cube Calculus is an algebraic model popular used to process and minimize Boolean functions. Cube Calculus operations are widely used in logic optimization, logic synthesis, computer image processing and recognition, machine learning, and other newly developing applications which require massive logic operations.

Cube calculus operations can be implemented on conventional general-purpose computers by using the appropriate "model" and software which manipulates this model. The price that we pay for this software based approach is severe speed degradation which has made the implementation of several high-level formal systems impractical.

A cube calculus machine which has a special data path designed to execute multiplevalued input, and multiple-valued output cube calculus operations is presented in this thesis. This cube calculus machine can execute cube calculus operations 10-25 times faster than the software approach.

For the purpose of ensuring the manufacturing testability of the cube calculus machine, emphasize has been put on the testability design of the cube calculus machine. Testability design and testability analysis of the iterative logic unit of the cube calculus machine was accomplished. Testability design and testability analysis methods of the cube calculus machine are weli discussed in this thesis. Full-scan testability design method was used in the testability design and analysis. Using the single stuck-at fault model, a $98.30 \%$ test coverage of the cube calculus machine was achieved. A novel testability design and testability analysis approach is also presented in this thesis. 


\title{
TESTABILITY DESIGN AND TESTABILITY ANALYSIS OF A CUBE CALCULUS MACHINE
}

\author{
by \\ LIXIN ZHOU
}

A thesis submitted in partial fulfillment of the requirements for the degree of

\author{
MASTER OF SCIENCE \\ in \\ ELECTRICAL AND COMPUTER ENGINEERING
}

Portland State University

1995 


\section{ACKNOWLEDGEMENTS}

I wish to express my profound gratitude to my adviser, Dr. Marek A. Perkowski, who patiently guided me through this endeavor, allowing me to be ambitious, creative and realistic.

I also wish to thank Dr. Y.C. Jenq and Dr. Maria Balogh for their valuable comments, suggestions and critiques on my thesis and their willingness to be in my thesis defense committee.

In addition, I express my appreciation to the previous students that have involved in the cube calculus machine project especially Luis Kida, Coen Engelbarts, and David Foote for their creative ideas in designing and building the cube calculus machine.

I especially thank Dr. Doug Hall and Mentor Graphics Corp. Dr. Doug Hall provided me reading materials when I started exploring design-for-test field. Mentor Graphics Corporation gave me the opportunity of learning their state-of-art design-for-test automation tools.

Finally, I would like to thank Shirley Clark and Laura Riddell for their continued support and help throughout my studying at Portland State University. 


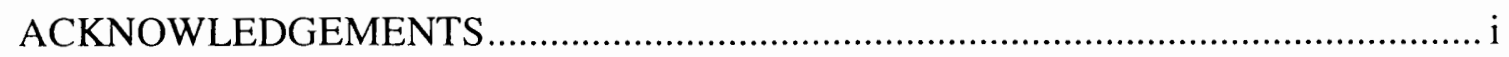

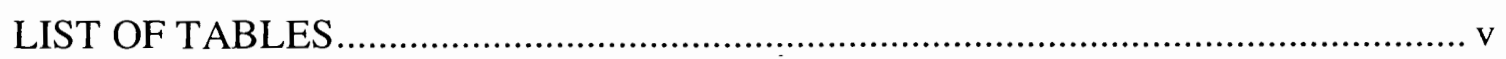

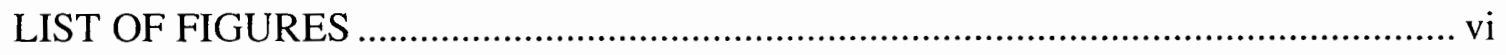

\section{CHAPTER}

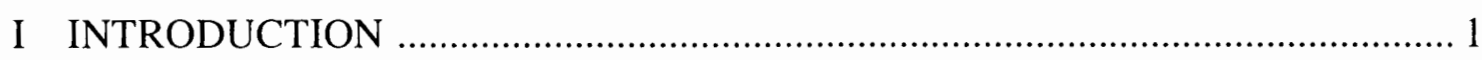

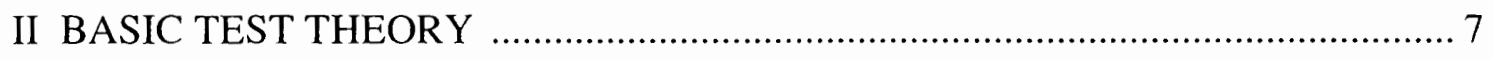

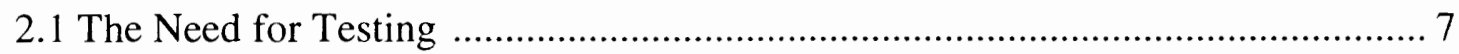

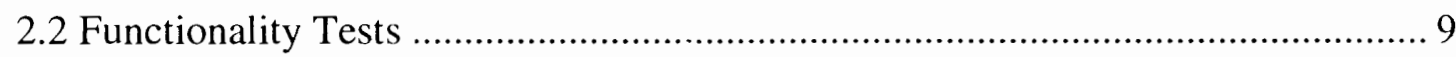

2.3 Manufacturing Defects and Tests .............................................................. 10

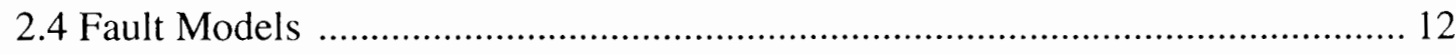

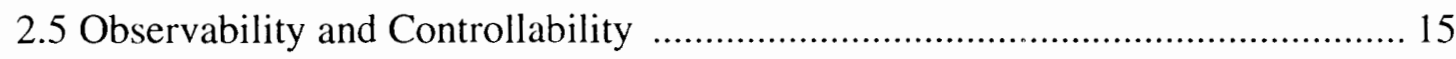

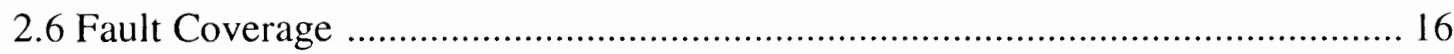

2.7 Automatic Test Pattern Generation (ATPG) ……….................................... 16

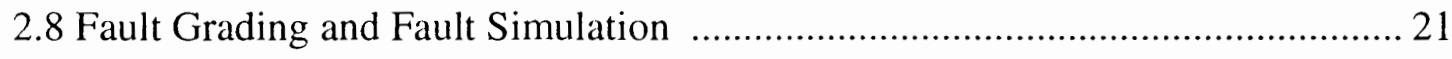

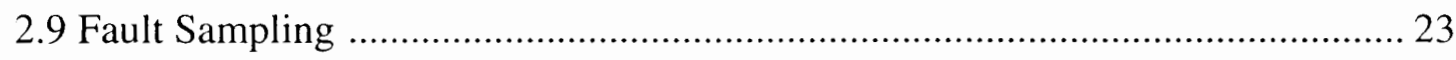

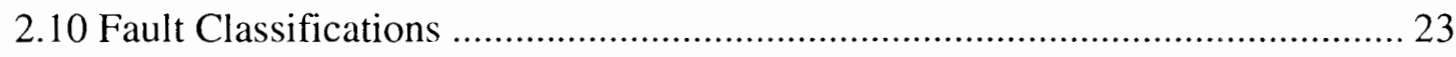

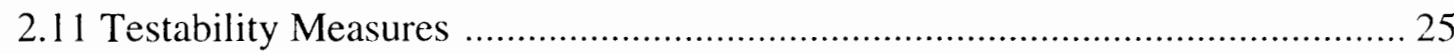

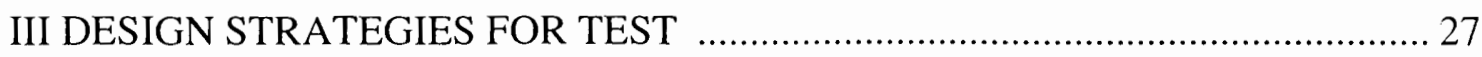

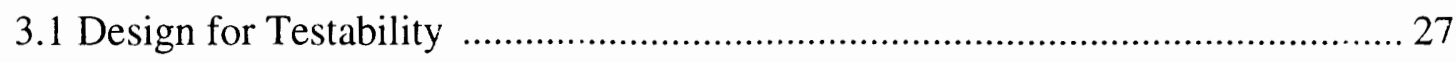

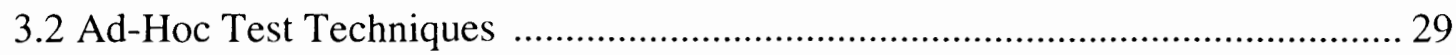

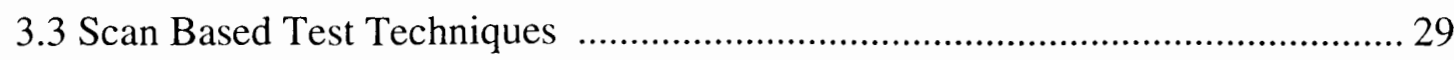




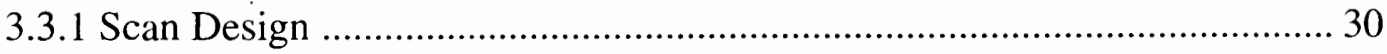

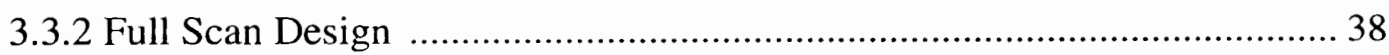

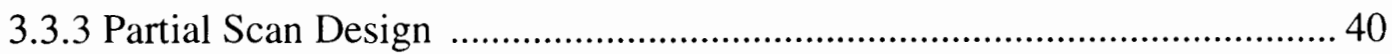

3.3.4 ATPG with Full Scan and Partial Scan ..................................................... 41

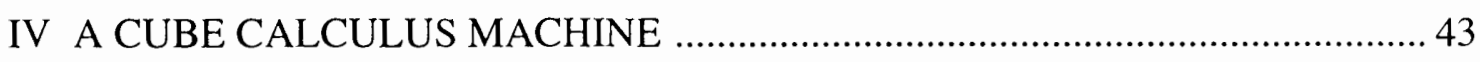

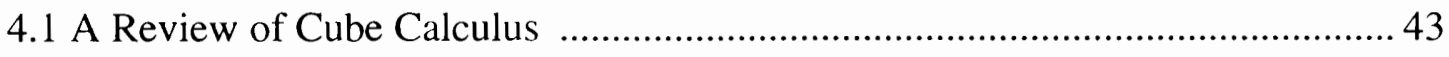

4.2 A Review of Cube Calculus Operations …………........................................... 46

4.2.1 Simple Combinational Operations ........................................................... 46

4.2.2 Sequential Cube Calculus Operations …………........................................ 47

4.2.3 Complex Combinational Operations …………………………….............. 54

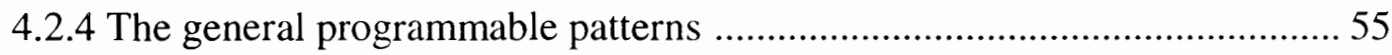

4.3 The idea of the Cube Calculus Machine (CCM) Architecture ............................ 56

4.4 The Concepts of Ring of Processors and Interlock Mechanism ......................... 59

4.5 The Block Level Description of the Architecture .............................................. 62

4.6 The Detailed Description of the Implementation of the Iterative Cell (IT) ......... 68

4.6.1 Iterative Signal in the Block Structure of IT Cells .......................................68

4.6.2 The Structure of a Single IT Cell .......................................................... 72

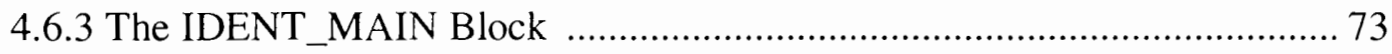

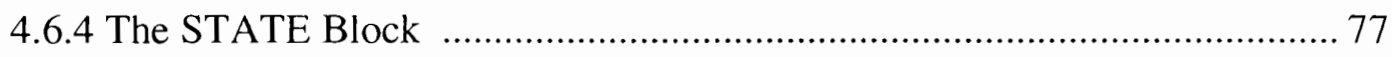

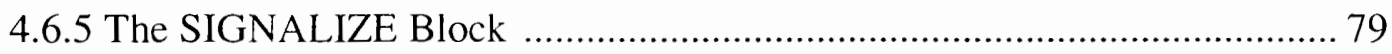

V TESTABILITY DESIGN AND TESTABILITY ANALYSIS …......................... 82

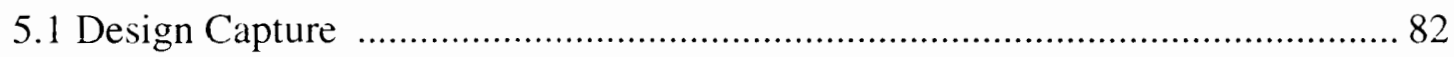

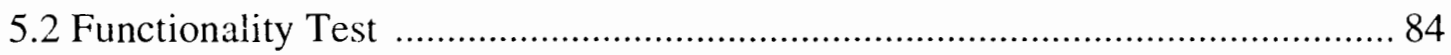

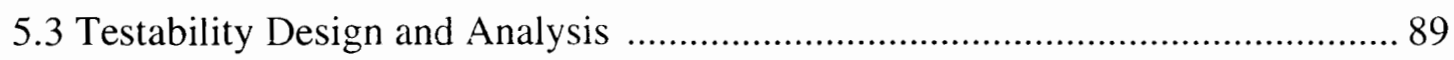

5.3.1 Fault Model and Design for Test Strategy ................................................ 90

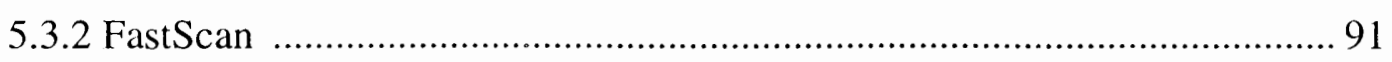

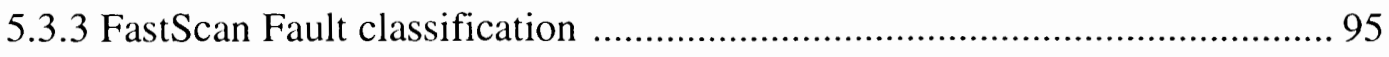




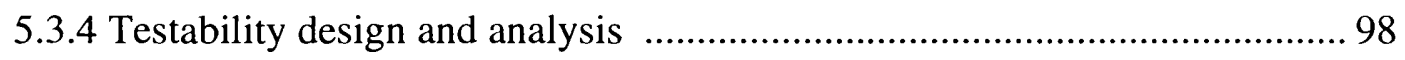

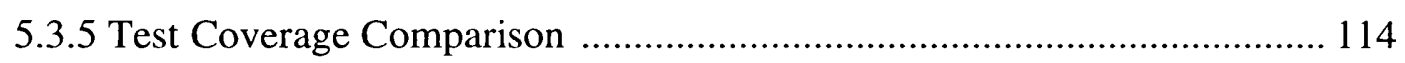

5.3.6 An Overview of Testability Design and Analysis on the CCM ............... 114

5.4 Verification of the Design's Functionality after Scan Insertion ........................ 115

5.5 Generation of a VHDL Model for the ILU ..................................................... 117

VI CONCLUSIONS AND FUTURE WORK ………........................................ 118

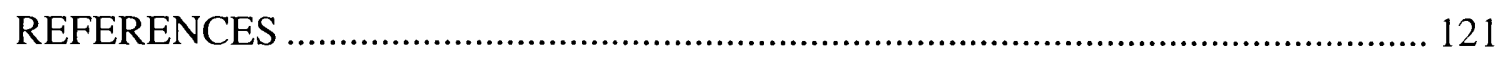

APPENDIXS

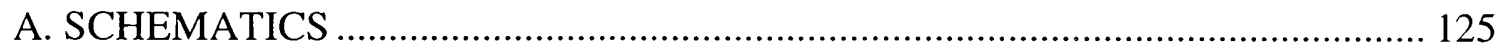

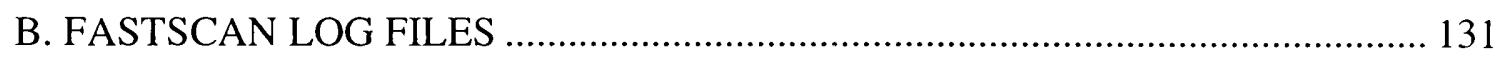




\section{LIST OF TABLES}

Table 1: Node-vector Summary of D Algorithm (Figure2) 18

Table 2: Cube calculus operations ........................................................................ 58

Table 3: Cube calculus operations on bits ........................................................59 


\section{LIST OF FIGURES}

page

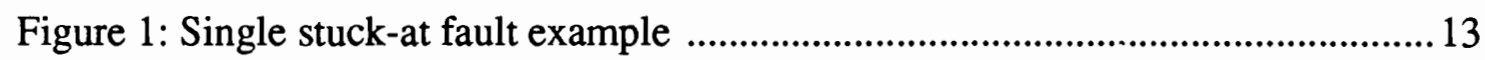

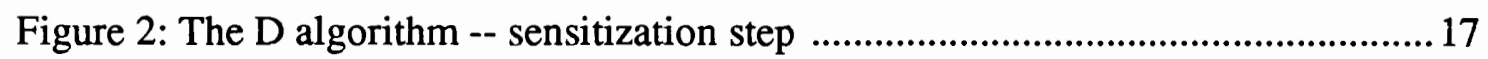

Figure 3: Reconvergent fan-out with D algorithm ................................................19

Figure 4: Test pattern generation with backtracking …..................................................20

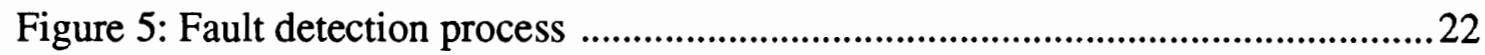

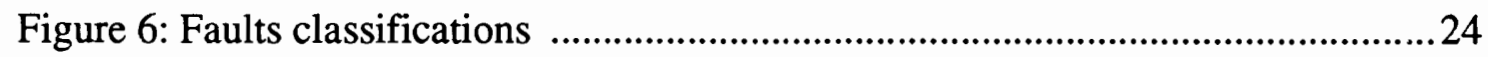

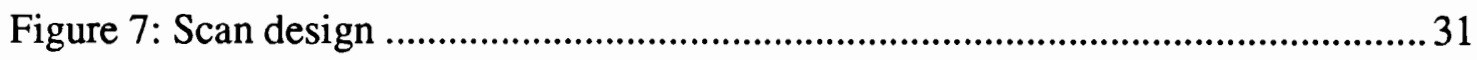

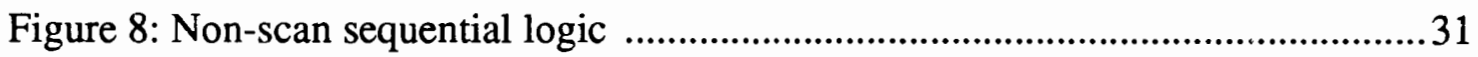

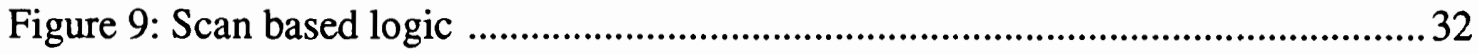

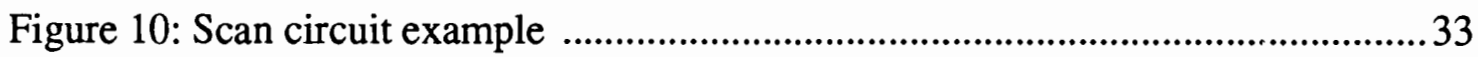

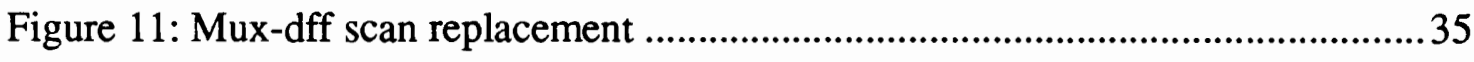

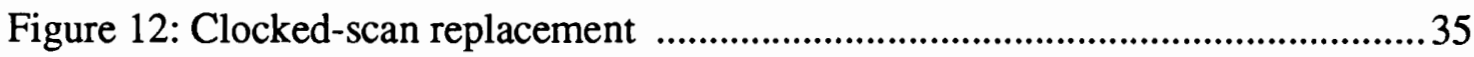

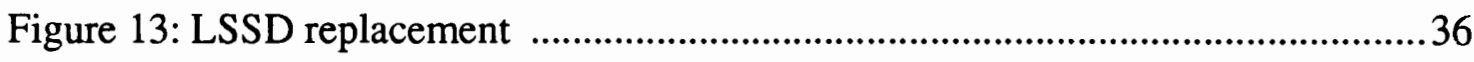

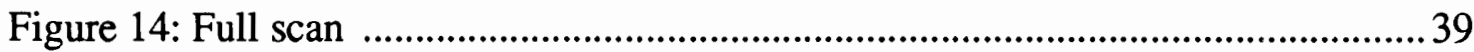

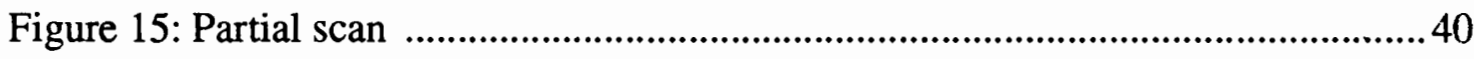

Figure 16: The three architectural models of a general-purpose computer ..................61

Figure 17: The block diagram of the CCM processor architecture ..............................63

Figure 18: The signals in IT .....................................................................................65

Figure 19: An example of a sequential operation .................................................69

Figure 20: Details of IT and interconnections of ITs in the ILU ..................................71

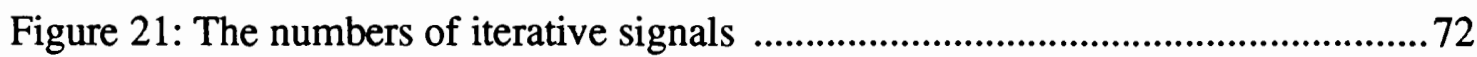

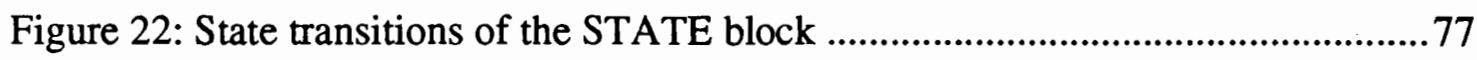

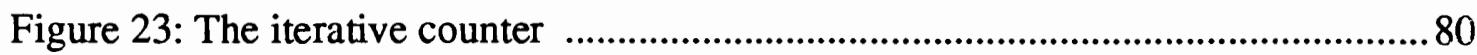

Figure 24: The hierarchical structure of the ILU …….................................................83 
Figure 25: Mux-dff scan replacement …………….......................................... 91

Figure 26: Example of possible detected fault …………...................................... 96

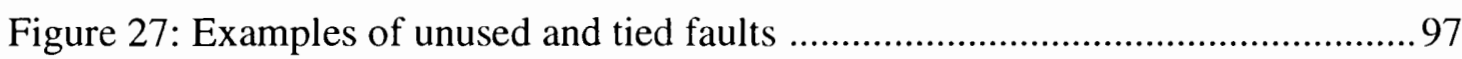

Figure 28: Examples of blocked and redundant faults ............................................. 98

Figure 29: Diagram of lower and higher level blocks ........................................... 100

Figure 30: FastScan report on the COUNTER block ............................................... 102

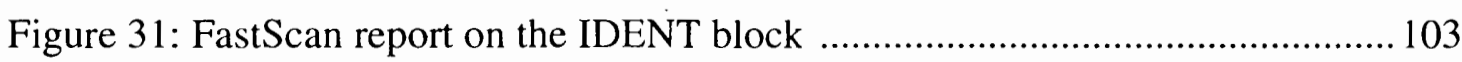

Figure 32: FastScan report on the STATE block ..................................................... 103

Figure 33: Example of ATPG untestable faults ....................................................... 104

Figure 34: ATPG untestable faults within the STATE block .................................... 105

Figure 35: dffs in STATE block before and after scan insertion ............................... 106

Figure 36: Test procedure file for the STATE_SCAN block .................................... 107

Figure 37: FastScan report file for STATE_SCAN block ....................................... 108

Figure 38: Tied faults in the STATE_SCAN block .................................................. 108

Figure 39: Detected by implication faults in the STATE_SCAN block .................... 109

Figure 40: FastScan report file for the IT block ....................................................... 110

Figure 41: A pair of ATPG untestable faults on an undriven input pin (I3) .............111

Figure 42: Illustration of the muxed REQUEST signal ........................................... 112

Figure 43: The test procedure file for the scan chain in the ILU .............................112

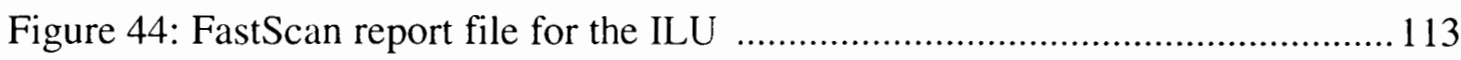

Figure 45: ATPG untestable faults in the ILU ………......................................... 114

Figure 46: FastScan report file on ILU without scan design ................................... 115

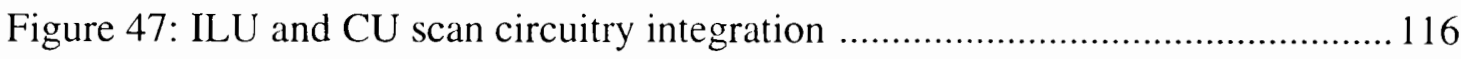

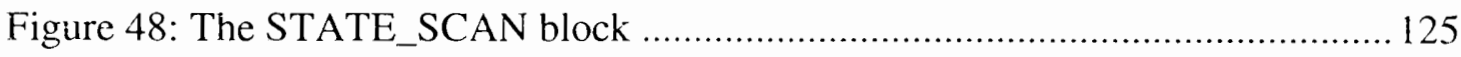

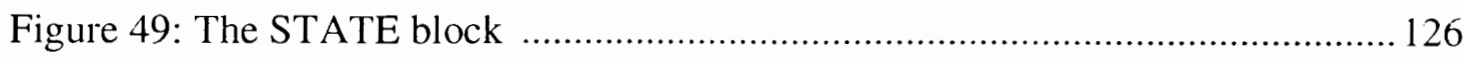

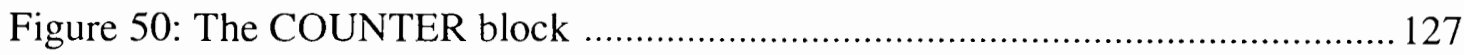

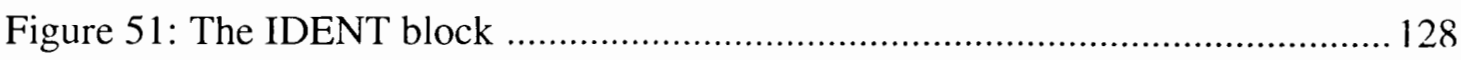

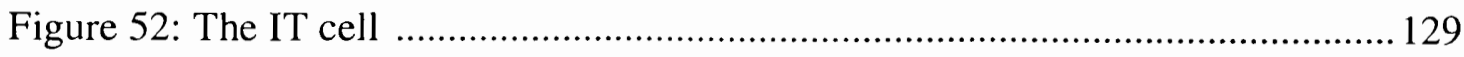

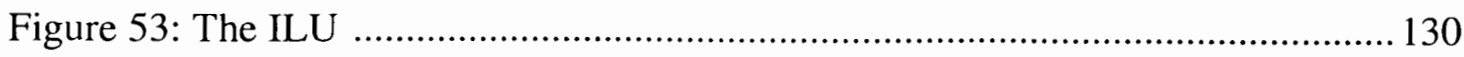




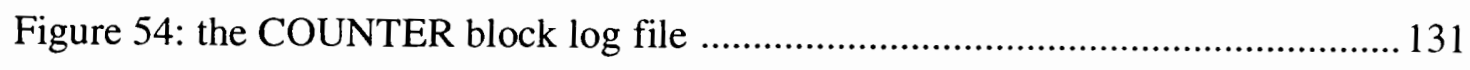

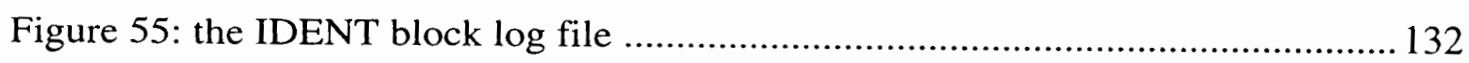

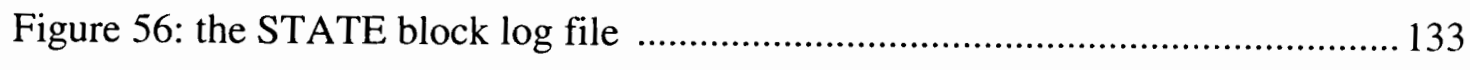

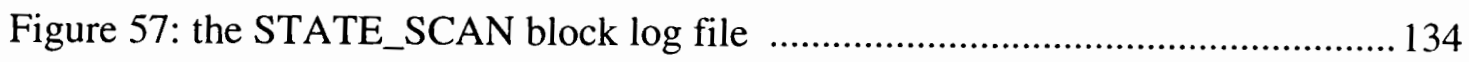

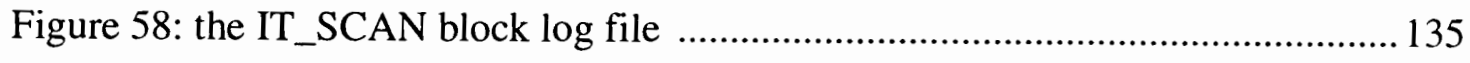

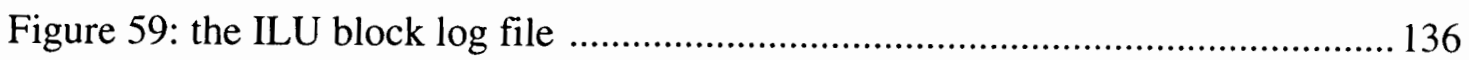




\section{CHAPTER I}

\section{INTRODUCTION}

Cube calculus is an algebraic model popularly used to process and minimize Boolean functions. Cube calculus operations are widely used in logic optimization, logic synthesis, computer image processing and recognition, machine learning, and other recently developed applications which require massive logic operations.

There is recently an intensive and growing interest in logic synthesis, both in theory and in the realization of practical design programs and systems of CAD tools. Many of the efficient modern logic synthesis programs use cube calculus to represent and process Boolean functions. This representation is used in U. C. Berkeley programs, including the well-known Espresso and MIS II, in programs from IBM (Brayton, Fleitcher, Roth), and many other.

Positional cube notation which is extended from cube calculus for a logic with multiple-valued inputs has been used for many two, three and many level Boolean minimizers, tautology and satisfiablity checkers, verifiers, programs for synthesis of mixed and fixed generalized Reed-Muller forms, generation of prime implicants, minimal and disjoint implicant, spectral transforms, and many other. It is also useful in programs that use other efficient representation of Bcolean functions, namely, the Binary Decision Diagrams.

Multiple-valued Cube Calculus (MVCC) is one of the most general internal representations of data in propositional logic, logic synthesis, logic design, logic programming, logic simulation and sequential evaluation of combinational logic, data-bases, and several areas of Artificial Intelligence (AI) and problem-solving. In logic design, it is pri- 
marily used for the minimization of PLAs that have decoders on the inputs, minimization of multiple-output binary functions and state assignment of FSMs. A programmable Logic Array (PLA) with r-bit decoders directly realizes a sum-of-product expression of $2^{r}$-valued input, two-valued output function.

Generalized Multiple-valued Cube Calculus (GMVCC), an extension of MVCC, is even more powerful than MVCC because it can represent multiple-valued input, multiple-valued output logic, multiple-output relations, predicates and other data. This means that it can be used for real-time AI applications, image processing, fuzzy logic and logic programming.

Cube calculus operations can be implemented on conventional arithmetic computers by using the appropriate "model" and software which manipulates this model.

In the architectures of conventional computers, the control is usually located in the program that is stored in RAM. This results in a considerable control overhead, since the instructions have to be fetched from RAM. If an algorithm contains loops, the same instructions have to be fetched many times. This makes the memory interface the bottleneck of these architectures, especially when the memory bus is not as fast as the internal processor bus.

The Arithmetic Logic Unit (ALU) in conventional computer architectures can only compute arithmetic and simple logic functions such as AND, OR, addition and shifting. With this set of basic functions, it is possible to perform many, more complicated operations and thus realize a general purpose computer. For many applications, however, this method results in slow and highly inefficient operation. For instance, to calculate the consensus of two cubes, the ALU must execute a long series of shifts and ANDs. Cube calculus operations implemented in this software approach suffer a severe speed degradation. This makes the implementation of several high-level formal systems impractical.

The slow processing speed of the cube calculus operations is a bottleneck for the realtime applications of many state-of-the-art logic synthesis and logic verification programs. 
There are two approaches that can be used to improve the processing speed of cube calculus operations. One is to design special hardware which implements the specific operations required by the cube calculus operations. Examples of this methods are math, DSP, and image processing co-processors. The other is to execute operations in parallel on multiple processors.

The first machine which realized a small subset of cube calculus operations in hardware was the Boolean Analyser (BA) of Antonin Svoboda [28] [29] which was used mainly to generate prime implicants, solve set covering problems, and Boolean equations. In 1985 a paper [26] describes a PAL-based coprocessor for checking logical tautology in order to speed-up two-level Boolean minimizers like MINI. In the same year, a paper [16] introduces a machine that is able to perform many operations used in logic synthesis and is optimized particularly for the minimization of satisfiablity formulas. Ulug [30] [32] [34] proposed an extended cube calculus machine for resolution-based theorem-proving with applications in real time $\mathrm{AI}$ and data-bases.

A cube calculus machine which has a special data path designed to execute GMVCC operations is presented in this thesis. This cube calculus machine can execute cube calculus operations 10-40 times faster than the software approach [3].

Most of the commonly used cube calculus operations have been implemented on our cube calculus machine. They are: supercube, intersection, prime, crosslink, sharp, disjoint sharp, symmetric consensus, and asymmetric consensus.

The CCM architecture is based on an observation that many cube calculus operations can be described in a general format of equation. In this general format, the literals of the operand cubes are divided into three types of literals, according to the relation of the literals of the operand cubes. This relation is typical of certain cube calculus operations. As the resultant cubes are created, the literals of the operand cubes go through a sequence of literal types. In this process, the borders between the different literals types propagate through all the literals. The value of a literal in the resultant cube not only depends on the corresponding literals in the operand cubes, but also on the current type of these literals. 
This general format of equation is implemented in the Iterative Logic Unit (ILU) of the CCM architecture. The ILU consists of an array of Iterative Cells (IT). Each IT can calculate 2 bits of the resultant cube. An IT contains a state machine to keep track of the current type of literal that is being processed. The value of the resultant literal is not only calculated from the operand literals, but also from the current internal state of the state machine. A considerable part of the control of the CCM is implemented in its data path, which is the ILU. Once the ILU has received a cube calculus operation, the only control that it need from the Control Unit (CU) of the CCM is the clock signals for the output of the resultant cubes.

In CCM architecture, most of the control is implemented in the data path. Once an instruction is loaded into the CCM, the host computer only needs to write the operand cubes to the CCM and read the resultant cubes from the CCM.

The CCM processor is optimized to execute cube calculus operations effectively. It can generate one resultant cube of a cube calculus operation in one clock cycle. No empty cubes are generated, which means that the output of resultant cubes doesn't have any irregularities.

One version of the cube calculus machine has been implemented by David Foote on Xilinx FPGAs. Results has shown that cube calculus operations executed on the FPGA cube calculus machine are 10-25 times faster than cube calculus operations executed by using software approach on conventional computer [3]. The larger the input cubes, the more speed gain can be obtained by using cube calculus machine. Application Specific Integrated Circuits (ASICs) usually have better timing performance than FPGAs. We can expect faster processing speed of cube calculus operation using ASIC cube calculus machine.

The running speed of many software applications which requires massive cube calculus operations can be significantly increased by using cube calculus machine as a cube calculus hardware accelerator. Examples of these software applications are logic synthesis programs, logic verification programs, image processing programs, machine learning programs, and much more. 
The first version of the cube calculus machine was introduced by Luis S. Kida and Dr. Marek Perkowski as a general purpose logic computer.

The author was actively involved in the developing of the second version of the cube calculus machine. The author has participated in the design and simulation of this version as a member of a group in Dr. Marek Perkowski's Computer Architecture for Robotics. The personal contributions of the author included improvement of the ILU equations and the special testing circuit for testing the functionality of a FPGA cube calculus machine was developed by the author.

It is our intention to manufacture the cube calculus machine as ASICs in the future. Due to the complexity of the manufacturing process not all die on a wafer correctly operate. Small imperfections in starting material, processing steps, or in photomasking may result in bridged connections or missing features. These manufacturing defects could cause the functional failure of a chip. Steps must be taken to ensure manufactured chips are testable in order to filter chips with manufacturing defects. This need leads to the effect of testability design and testability analysis of our cube calculus machine.

The design and analysis of a highly testable cube calculus machine is presented in this thesis.

Chapter II and III serves as introduction of design-for-test. Chapter 2 gives and overview of the basic test theory. Chapter 3 introduces the concept of design-for-test with emphasize on scan design techniques which was used in the testability design of the ILU. They should be helpful to other students involved in the future development of the cube calculus machine, especially the testability design and analysis of the control unit of the cube calculus machine.

Chapter IV presents an overview of cube calculus and cube calculus operations. Detailed functional and structural description of the ILU is also given in this chapter.

Chapter V shows the steps and the methods of the testability design and analysis on the ILU. A novel testability design and testability analysis approach is proposed here. A brief introduction of FastScan which is the ATPG tool used to help the testability 
analysis is also given in chapter.

Chapter VI gives a conclusion of the tasks that were accomplished in this thesis and a list of future work that lead to a successful ASIC cube calculus machine. 


\section{CHAPTER II}

\section{BASIC TEST THEORY}

\subsection{The Need for Testing}

While most problems in VLSI design have been reduced to algorithms in readily available software, the responsibilities for the various levels of testing and testing methodology can be a significant burden on the designer.

When a chip is fabricated, it is tested. Chips that pass the testing process are then sold. A certain percentage of these parts will fail. Chips that fail the testing process are rejected. However, some faulty devices may pass through the testing process due to poor quality or incomplete tests. The number of defective parts sold as "good" is known as the defect level (DL). The defect level is usually measured in defective parts per million (DPM).

Test coverage is a measure of the number of faults detected by the testing process divided by the total number of faults possible in the device. Test coverage of $100 \%$ means that all the possible faults are detected by the test. Test coverage near $100 \%$ procedures a defect level near 0 . Low test coverage procedures a higher defect level. Thus it is very important to have high test coverage.

Having a low number of defective parts per million is crucial. Shipping a product with high DPM can prove very costly to the company. This is the motivation for creating test sets with high test coverage.

Different types of testing occur at various stages in product development. The cost of finding mistakes grows exponentially during the product development phases.

Analysis of the design specification prior to the actual design process is the least expensive time to find problems with the design. At this point, the specification can 
easily be changed.

During the design phase, a design model is created and analyzed for proper functionality. If mistakes are found, the design model is modified. At this point, it is getting somewhat more costly to find mistakes. Design modification and analysis continues until no more mistakes in functionality are found. Once the design is proven functionally correct, it is manufactured.

After the design is manufactured, the physical device is tested. Mistakes found at this point can be relatively costly. Chips cannot be repaired, so rejected parts must be disposed of and therefore generate no revenue for the company.

Problems discovered in the field are the costliest of all. This indicates that a part considered "good" is actually faulty. This situation indicates poor quality testing and can prove extremely costly. The cost of defective parts in critical devices can often be immeasurable.

Tests may fall into two main categories. The first set of tests verifies that the chip performs its intended function; that is, that it performs a digital filtering function, acts as a microprocessor, or a communicates using a particular protocol. In other words, these tests assert that all the gates in the chip, acting in concert, achieve a desired function. These tests are usually used early in the design cycle to verify the functionality of the circuit. These will be called functionality tests in this thesis. They may be lumped into the verification activity. The second set of tests verifies that every gate and register in the chip functions correctly. These tests are used after the chip is manufactured to verify that the silicon is intact. They will be called manufacturing tests in this thesis. In some cases these two sets of tests may be one and the same, although the natural flow of design usually has a designer considering function before manufacturing concerns.

It is interesting to note that of most first-time failures of silicon, it is the functionality of the design that is to blame which means that the chip does exactly what the simulator said it would, but for some reason (almost always a human error) that function is not what the rest of the system expects. 


\subsection{Functionality Tests}

Functionality tests are usually the first test a designer might construct as part of the design process. Does this adder add? Does this counter count? Does this state machine yield the right outputs at the right clock cycles?

For most systems, functionality tests involve proving that the circuit is functionally equivalent to some specification. That specification might be a verbal description, a plain-language textual specification, a description in some high-level computer language such as $\mathrm{C}$, Pascal or in a hardware description language such as VHDL, or Verilog, or simply a table of inputs and the required outputs. Functional equivalence involves running a simulator at some level on the two descriptions of the chip (say, one at the gate level and one at a functional level) and ensuring for all inputs applied that the outputs are equivalent at some convenient check points in time. The most detailed check might be on a cycle-by-cycle basis.

Functional equivalence may be carried out at various levels of the design hierarchy. If the description is in a behavioral language (such as the last two categories mentioned), the behavior at a system level may be verifiable. For instance, in the case of a microprocessor, the operating system might be booted and key programs might be run for the behavioral description. However, this might be impractical for a gate-level model and extremely impractical for a transistor-level model because of the long simulation times. The way out of this is to use the hierarchy inherent within a system to verify chips and modules within chips. That, combined with well-defined modular interfaces, goes a long way in increasing the likelihood that a system composed of many VLSI chips will be first-time functional. At the lowest levels of the hierarchy, timing tests must be run to validate that a particular function is achieved at a given clock rate.

There is no good theory on how to ensure that good functional tests be written. The best way is to simulate the chip or system as closely as possible to the way that it will be used in the real world. Often this is impractical due to slow simulation times and very long verification sequences. One approach is to move up the simulation hierarchy 
as modules become verified at lower levels. For instance, the gate-level adder and register modules in a video filter might be replaced by functional models and then the filter itself might be replaced by a function model. At each level, small tests are written to verify the equivalence between the new higher-level functional model and the lowerlevel gate or functional level. At top level, the filter functional-model may be surrounded with a software environment that models the real world that uses the filter. Finally, if enough time is available, all or part of the functional test may be applied to the gate level and even the transistor level, if transistor primitives have been used. One approach that is becoming more popular and feasible is to model chips as collections of reprogrammable gate arrays. Commercial hardware is available to aid this activity.

\subsection{Manufacturing Defects and Tests}

Due to the complexity of the manufacturing process not all die on a wafer correctly operate. Small imperfections in starting material, processing steps, or in photomasking may result in bridged connections or missing features.

Whereas functionality tests seek to verify the function of a chip as a whole, manufacturing tests are used to verify that every gate operates as expected. The need to do this arises from a number of manufacturing defects during chip fabrication or during accelerated life testing (where the chip is stressed by over-voltage and over-temperature operation). Typical defects include:

- layer-to-layer shorts (i.e., metal to metal).

- discontinued wires (i.e., metal thins when crossing vertical topology jumps).

- thin-oxide shorts to substrate or well.

These in turn lead to particular circuit defects, including:

- nodes shorted to power or ground.

- nodes shorted to each other.

- inputs floating/outputs disconnected. 
Tests are required to verify that each gate and register is operational and has not been compromised by a manufacturing defect. Tests are normally carried out at the wafer level to cull out bad die, and then on the package's parts. The length of the tests at the wafer level might be shortened to reduce test time based on the experience with the test sequence.

Apart from the verification of internal gates, $\mathrm{L} / \mathrm{O}$ integrity is also tested through completing the following tests:

- I/O-level test (i.e., checking the noise margin for TTL, ECL, or CMOS I/O pads).

- at-speed test.

- IDDQ test ( $V_{\mathrm{DD}}$ supply current Quiescent).

The last of these tests checks the leakage if the circuit is composed of complementary logic. Any value markedly above the expected value for a given wafer normally indicates an internal shorting failure (or very bad leakage).

In general, manufacturing test generation assumes that the circuit/chip functions correctly, and ways of exercising all gate inputs and of monitoring all gate outputs are required.

To illustrate the difference between a functional test and a manufacturing test, consider the testing of a microprocessor at a functional level, which might be the first concern of the designer. To test any instruction, a sequence of instructions that use that instruction might be used (i.e., does the ADD instruction add?). While this might prove that the control logic that yields that instruction is intact, it does not, for instance, prove that the instruction works for all the possible addresses and data. At this level of test it is assumed that the adders, muxes, gates, and registers in the microprocessor's data path operate correctly.

Tests that exercise all bits in the data path have to be written to verify the chip at the manufacturing level. These tests might include a test to check that registers can store a 1 and a 0 and a test that exercises each bit in any adder and ensures that the carry chain is not broken (i.e., does the adder add for all inputs?). The inputs have to be chosen carefully to check for all possible manufacturing defects. The manufacturing tests may 
be the only tests applied to a microprocessor prior to its being placed in a socket and booted.

\subsection{Fault Models}

In order to abstract the behavior of the manufacturing defects at the logical gate level, the fault model is used to cover the underlying physical effects.

Different test types target different types of models. The most common fault model types include stuck-at, IDDQ, and transition. These fault models are all based on gatelevel netlist. They model the effects of opens and shorts of the metal layers of the actual chip on the netlist.

\section{Single Stuck-At Fault Model}

The single stuck-at fault model is the most common fault model used in fault simulation. Years of research has proven it is effective in finding many common defect types and it has become an industry standard.

The stuck-at fault model models behavior that occurs if the terminals of a gate are stuck at either high (stuck-at-1) or low (stuck-at-0) voltage levels. Most physical defects that occur exhibit behavior that makes a node appear to be stuck at power or ground.

Note that a single stuck-at fault does not simply model a fault to power or ground. Instead, it models the change in the function of the cell with a failure, which is the same as if one of its terminals is always stuck at logic 1 or 0 .

To detect a stuck-at fault, a test pattern need to be applied to the primary inputs of a circuit and measure the difference (between the good and faulty circuit) at the primary outputs.

Figure 1 shows a stuck-at fault example. In this example, if input $a$ on the primitive NAND gate is stuck-at 1, a 0 applied to the input can not change $a$ to 0 . 


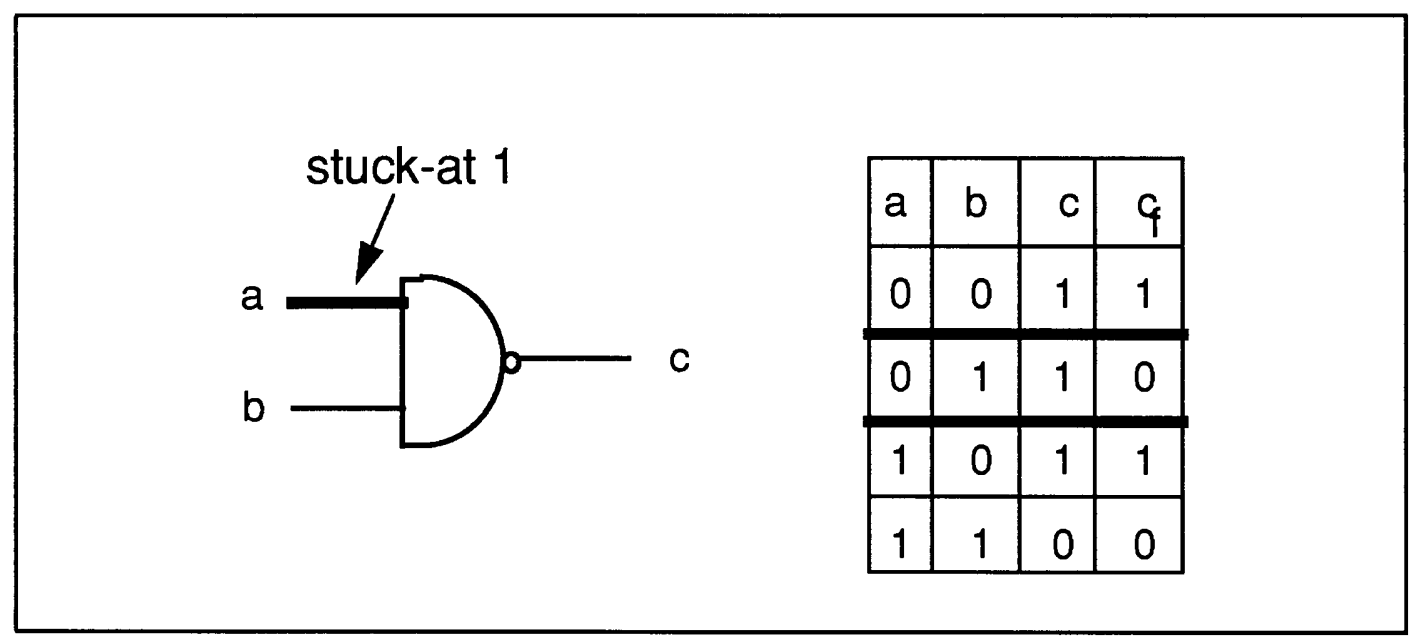

Figure 1: Single stuck-at fault example

To detect this stuck-at 1 fault at pin $a$, first control the fault by forcing the fault site to its unfaulty state $(a=0)$. Then observe the fault by propagating it through the NAND gate to the observe point, $c$. The sensitizing value for a NAND gate is 1 , so set $b=l$ to observe the fault effect. If $c$ remains 0 , the fault is detected. Thus, $a=0$ and $\mathrm{b}=I$ is a set of test vector to detect $a$ stuck-at 1 .

\section{IDDQ Testing and the Pseudo Stuck-At Fault Model}

IDDQ testing ( $V_{\mathrm{DD}}$ supply current Quiescent) is becoming increasingly more important in the test field. There are a number of different physical defects which cannot be detected by the traditional stuck-at model. If we can measure the current of a device, we can detect a number of these faults. IDDQ testing can detect manufacturing defects that the traditional stuck-at fault model cannot, such as CMOS transistor shorts. Thus, combining stuck-at fault testing with IDDQ testing can greatly improve test coverage of a design.

As the name suggests, IDDQ testing measures design current, and rejects a part if the measured current is over a threshold current level. Because it is costly and impractical to monitor current for every test vector in the set, a small, efficient set of test patterns 
can typically be selected or generated for IDDQ testing to supplement the stuck-at set. Using IDDQ testing, there may be situations where high current occurs legally. Also, we only get a handful of current measures (depending on the vendor) for IDDQ testing. Despite the few current measures, we can often get high test coverage with this type of testing.

IDDQ testing can be used with various types of fault models. However, the pseudo stuck-at model is the only one discussed here. The intent of the pseudo stuck-at model is to detect internal transistor shorts, as well as "hard" stuck-ats. It is based on the principle that current flows when two nodes are connected and driven to different values. Unlike stuck-at faults which require fault effects to be propagated to the circuit outputs for detection, pseudo stuck-at faults make a current observation at the cell output where the current can be directly monitored through $V_{\mathrm{DD}}$ supply current.

\section{Transition Fault Model}

Transition faults model large circuit delays, which are the result of slow transistor transitions. The transition fault model is a little more sophisticated than the stuck-at fault model. The transition fault model behaves as a stuck-at fault, for a certain period of time. The slow-to-rise transition fault models a pin that is defective because it is slow to rise from a 0 to a 1 . The slow-to-fall transition fault models a pin that is slow to change from a 1 to a 0 .

Because a transition fault models a pin that appears defective (as a stuck-at) for some length of time, it requires two test vectors for detection. The first vector is the initialization vector. It places the initial transition value at the fault site. The second vector is the transition vector. It is identical to the vector that would detect the associated stuck-at fault. It places the final transition value at the fault site.

Because of pattern dependency (two sets of test patterns are needed to detect a transition fault), transition fault coverage typically does not exceed $70 \%$. Thus, this type of testing should be combined with stuck-at or other types of testing to achieve higher coverage. 
In the manufacturing environment, use of these vectors on test equipment requires proper user-supplied timing. The timing of strobes can be difficult to set up. If the timing is set wrong, the transition fault patterns still detect the stuck-at faults.

\subsection{Observability and Controllability}

The observability of a particular internal circuit node is the degree to which one can observe that node at the outputs of an integrated circuit (i.e., the pins). This measure is of importance when a designer desires to measure the output of a gate within a larger circuit to check that it operates correctly. Given a limited number of nodes that may be directly observed, it is the aim of well-designed chips to have easily observed gate outputs, and the adoption of some basic test design techniques can aid tremendously in this respect. Ideally, one should be able to observe directly or with moderate indirection (i.e., one may have to wait a few cycles) every gate output within an integrated circuit. While at one time this aim was hindered by limited gate count processes and a lack of design methodology, current design practices and processes allow one to approach this ideal.

The controllability of an internal circuit node within a chip is a measure of the ease of setting the node to a 0 or 1 state. This measure is of importance when assessing the degree of difficulty of testing a particular signal within a circuit. An easily controllable node would be directly settable via an input pad. A node with little controllability might require many hundreds or thousands of cycles to get it to the right state. Often one finds it impossible to generate a test sequence to set a number of poor controllable nodes into the right state. It should be the aim of a well-designed circuit to have all nodes easily controllable. In common with observability, the adoption of some simple design for test techniques can aid tremendously in this respect. 


\subsection{Fault Coverage}

A measure of goodness of a test program is the amount of fault coverage it achieves; that is, for the vectors applied, what percentage of the chip's internal nodes were checked. Conceptually, the way in which the fault coverage is calculated is as follows. Each circuit node is taken in sequence and held to 0 (stuck-at-0), and the circuit is simulated, comparing the chip outputs with a known "good machine" -- a circuit with no nodes artificially set to 0 (or 1 ). When a discrepancy is detected between the "faulty machine" and the good machine, the fault is marked as detected, and the simulation is stopped. This is repeated for setting the node to 1 (stuck-at-1). In turn, every node is stuck at 1 and 0 , sequentially. The total number of nodes that, when set to 0 or 1 , do result in the detection of the fault, divided by the total number of nodes in the circuit, is called the percentage-fault coverage.

The above method of fault analysis is called sequential fault grading. While this might be practical for small circuits, or by using hardware simulation accelerators on medium circuits, the time to complete the fault grading may be very long. On average $K N$ cycles need to be simulated, where $K$ is the number of nodes in the circuit and $N$ is the length of the test sequence, assuming that, on an average, N/2 cycles are needed to detect each fault.

To overcome these long simulation times many ingenious techniques have been invented to deal with fault simulation.

\subsection{Automatic Test Pattern Generation (ATPG)}

Historically in the IC industry, designers designed circuits, layout drafts-people completed the layout, and the test engineer wrote the tests. In many ways, the test engineers were reverse-engineering the circuits to create tests that would test the circuits in an adequate manner. 
Manual generation of test vectors is a serious bottleneck for time-to-market, due to:

- Increasing gate count in a design.

- Ratio of $\mathrm{I} / \mathrm{O}$ pins to gates decreasing makes the nodes in a circuit harder to control and observe.

- Increasing demand for product quality, which increases test coverage requirements. Top-down design creates a test crisis. Designer has little knowledge of gate structure because of using synthesis tools. Manual generation of test vectors becomes infeasible.

To deal with this burden, methods for automatically generating tests have been invented. This is known as Automatic Test Pattern Generation.

Most ATPG approaches have been based on simulation. A five-valued logic [12] form is commonly used to implement test generation algorithms. This consists of the states $1, O, D, \bar{D}$, and $X .0$ and 1 represent logical zero and logical one respectively. $X$ represents the unknown or DON'T-CARE state. $D$ represents a logic 1 in a good machine and a logic 0 in a faulty machine while $\bar{D}$ represents a logic 0 in a good machine and a logic $l$ in a faulty machine.

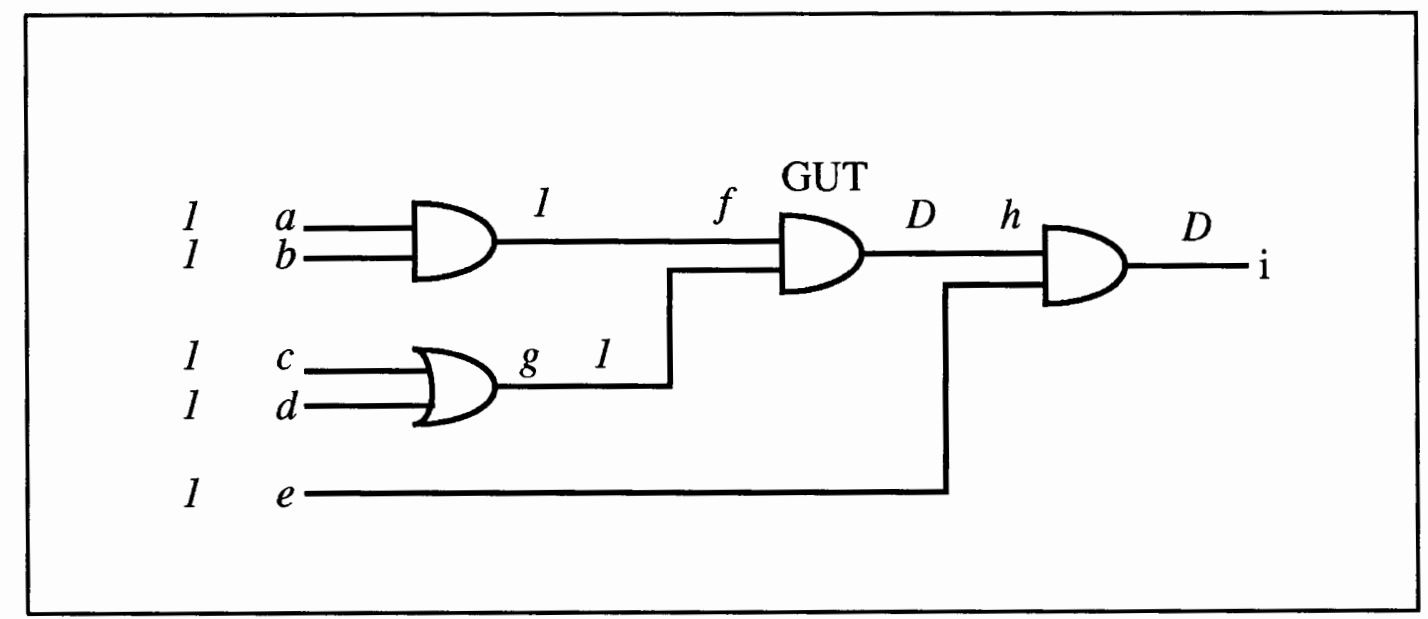

Figure 2: The D algorithm -- sensitization step 
We can examine the use of these five-valued logic by considering the circuit shown in Figure 2 where an stuck-at-0 fault is to be detected at node $h$. Thus node $h$ would have value $D$. There are two objectives. The first is to propagate the $D$ on node $h$ to one or more primary output. A primary output is a directly observable signal, such as a pad or, as we will see later, a scan output. This path to the primary output is called the sensitized path. The second object is to set node $h$ to state $D$ via a pad or some other means. The gate driving node $\mathrm{h}$ is the Gate Under Test (GUT). From node $\mathrm{h}$ we backtrack to the primary outputs $(a, b, c, d, e)$ to find the necessary input vector required to set node $h$ to a 1 . Because the gate driving node $h$ is an AND gate from the above definition (a $D$ is a $l$ in a good machine), both inputs $(f, g)$ have to be set to $l$ to set $h$ to 1 . Proceeding further toward the inputs, to assert node $f$ as a 1 , both nodes $a$ and $b$ have to be set to a 1. Because node $g$ is driven by an OR gate, either node $c$ or node $d$ need to be set to a $I$ to assert node $g$. Thus a vector $\{a, b, c, d\}$ of $\{1,1,1,0\}$ or $\{1,1,0,1\}$ is required to control node $h$. To observe that node g has been set to a $D$, input node e has to be set to a $l$. Thus the resultant test vector is $\{a, b, c, d, e\}=\{1,1,0,1,1\}$ or $\{1,1,1,0,1)$. If we are checking for an stuck-at- $I$ fault at node $h$, we must be able to set it to a 0 . By a similar reasoning. for the stuck-at- 0 case the test vector would be $\{a, b, c, d, e\}=\{0,1, X, X, 1\}$ or $\{1,0, X, X, 1\}$ or $\{0,0, X, X, 1\}$ or $\{1,1,0,0,1\}$. Similarly, for other nodes a summary of the vector is as in Table 1.

Table 1: Node-vector Summary of D Algorithm (Figure2)

\begin{tabular}{|c|c|l|}
\hline NODE & TEST & \multicolumn{1}{|c|}{ VECTOR $\{a, b, c, d, e\}$} \\
\hline \hline$h$ & S-A-0 & $\{1,1,0,1,1\},\{1,1,1,0,1\}$ \\
\hline$h$ & S-A-1 & $\{0,1, X, X, 1\},\{1,0, X, X, 1\},\{0,0, X, X, 1\},\{1,1,0,0,1\}$ \\
\hline$f$ & S-A-0 & $\{1,1,0,1,1\},\{1,1,1,0,1\}$ \\
\hline$f$ & S-A-1 & $\{0,0,0,1,1\},,\{0,0,1,0,1\}$ \\
\hline$g$ & S-A-0 & $\{1,1,0,1,1\},\{1,1,10,1\},\{1,1,1,1,1\}$ \\
\hline$g$ & S-A-1 & $\{1,1,0,0,1\}$ \\
\hline
\end{tabular}


The next step is to collapse the vectors into the least set that covers all nodes. A possible set is $\{1,1,0,1,1\},\{0,0,1,0,1\},\{1,1,0,0,1\}$.

The reason for using a five-valued logic is shown in Figure 3. Here an additional AND gate and INVERT gate have been added to the circuit. We can see that a fault at node $h$ is essentially unobservable. When an stuck-at- 0 fault is to be detected at node $h$, node $h$ would have value $D$. When input $e$ is set to a 1 , this fault effect can be observed at node $i$. Thus node $i$ has value $D$. At the same time, the fault effect on node $h$ has been propagated through the inverter. Node $k$ has value $\bar{D}$. For the last AND gate, since one of its inputs has value $D$ and the other has value $D$, the output of this AND gate, node $j$, will always be 0 . This means the effect of the stuck-at- 0 fault at node $h$ can not be propagated to the observe point of this circuit. This fault is unobservable. Same reasoning can be done for stuck-at-1 fault at node $h$. The stuck-at- 1 fault at node $h$ is also unobservable. This circuit suffers from what is called the reconvergent fan-out.

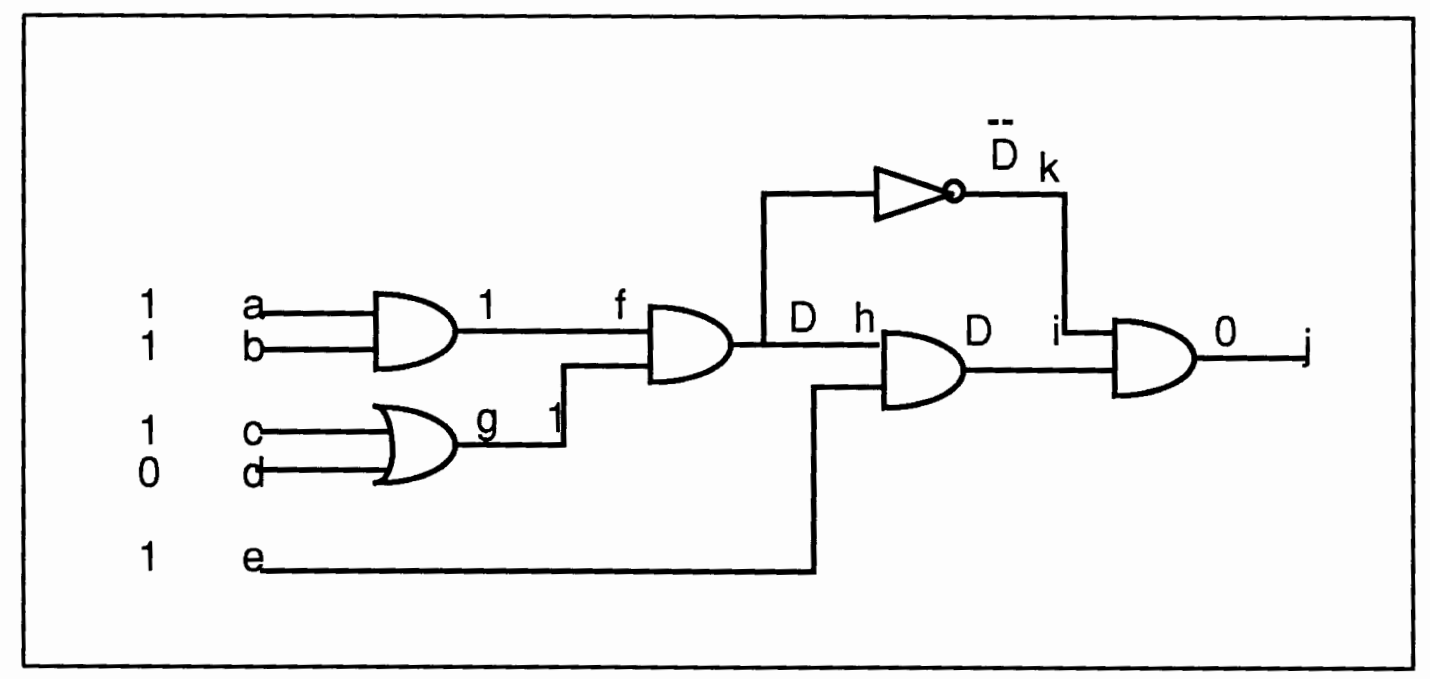

Figure 3: Reconvergent fan-out with $\mathrm{D}$ algorithm

The circuit in Figure 4 shows an example of test pattern generation with backtracking. 


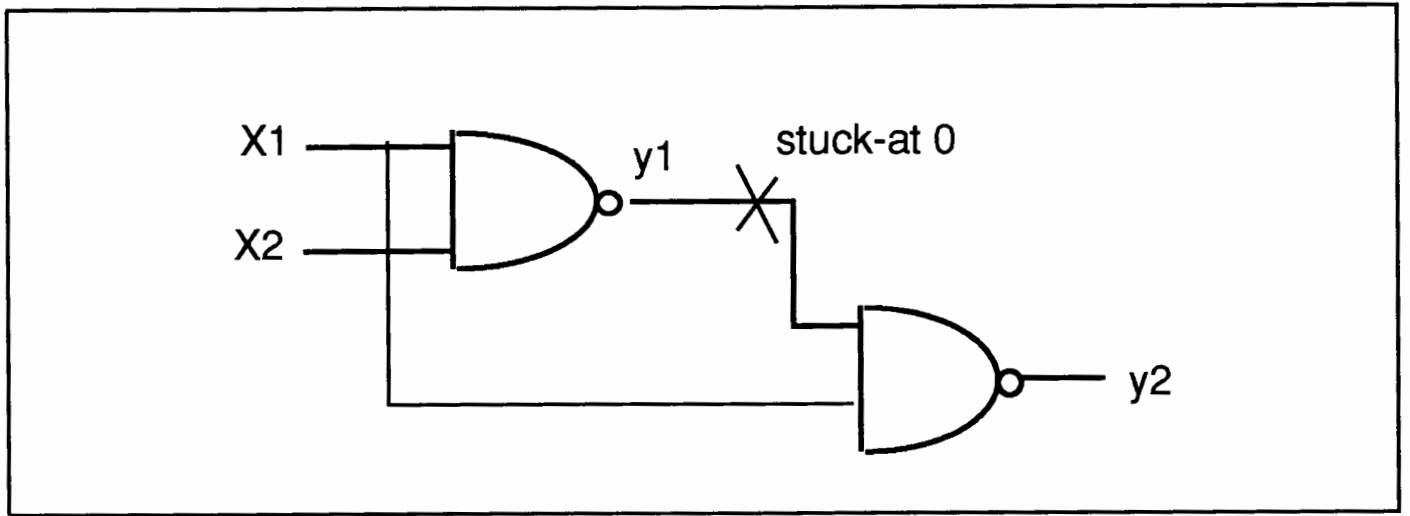

Figure 4: Test pattern generation with backtracking

The steps to find a test vector for $y l$ stuck-at 0 are:

1 . Set $y l$ to 1 .

2. Justify step 1 by setting $x l=0$.

3. Carry forward by implication of $x l=0$ that $y 2=1$.

4. Propagate value on $y l$ to $y 2$--Does not work!

5. Backtrack to step 3 and undo it.

6. Backtrack to step 2 and set $x 2=0$.

7. Propagate $y l$ forward by setting $x l=1$.

We have control over how many decisions (back-tracking attempts) the test generation tool is allowed to make in its processing. When the process must back-track, it is essentially aborting its current attempt. Thus, the number of back-tracking attempts allowed is called the abort limit.

The usual basis for manual generation of tests by test engineers and by many current automatic test pattern generation programs is the D-algorithm (DALG). PODEM[21] and PODEM-X[22] are improved algorithms that are more efficient than the original DALG[10]. Another ATPG algorithm is called FAN[9], and an improved efficiency algorithm dealing with tristate drivers, called ZALG, has been developed. Other works have concentrated on dealing at a module level rather than at a gate level. In principle, these algorithms start by propagating the $D$ value on an internal node to a primary output. This is called the D-propagation phase. The selection of which gates to pass 
through to the output is guided by a value of the observability index assigned to each gate. At any particular gate input, the gate with the highest observability value is selected. Once the D value is observable at a primary output, the next step is to determine the primary input values that are required to enable the fault to be observed and tested. This proceeds by backtracking from the faulted signal through the sensitized path and toward the primary inputs. The selection of which path to proceed along toward the inputs is aided by controllability indices assigned to nodes. This is known as backtrace step.

Controllabilities and observabilities can be assigned statically (that is, without regard to the logic state of the network) or dynamically (that is, according to the current state of the network). The SCOAP[14] algorithm is one method of assigning controllabilities and observabilities.

More recently, some authors have proposed the use of massively parallel computing methods for ATPG[27]. Methods have also been developed that model faults as changes to a Boolean network. Equivalence checking is used to prove that the two networks are not equivalent. These methods, when combined with random-fault generation and fault simulation, have demonstrate a great deal of success.

\subsection{Fault Grading and Fault Simulation}

Fault grading consists of two steps. First the node to be faulted is selected. Normally global nodes such as reset lines and clock lines are excluded because faulting them can lead to unnecessary simulation. A simulation is run with no faults inserted, and the results of this simulation are saved. Following this process, in principle, each node or line to be faulted is set to 0 and then to 1 and the test vector set is applied. If, and when, a discrepancy is detected between the faulted circuit response and the good circuit response, the fault is said to be detected and the simulation is stopped, and the process is repeated for the next node to be faulted. This process is illustrated in Figure 5. 


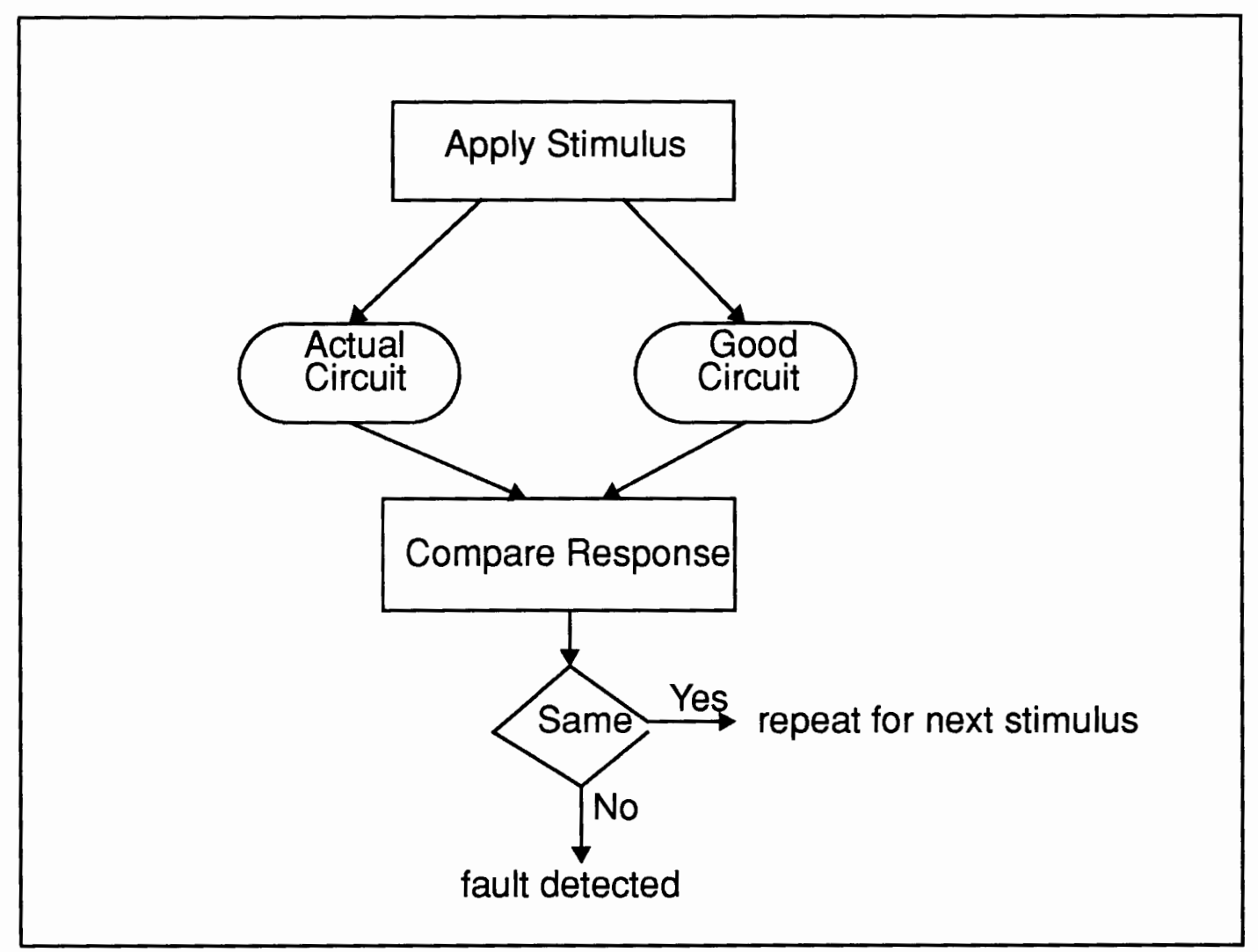

Figure 5: Fault detection process

If the number of the nodes to be faulted is $K$, and the number of test vectors is $N$, the number of simulation cycles, $S$, is approximately given by

$$
S=2 \frac{N}{2} K+N=K(N+1) \approx K N
$$

This serial fault simulation process is therefore running $K$ sets of the test vector set. With a small vector set, simple circuit, or very fast simulator, this approach is feasible. However, for large test sets and circuits, it is highly impractical.

To deal with this problem a number of ideas have been developed to increase the speed of fault simulation. Concurrent simulation is one of the ideas. 


\subsection{Fault Sampling}

Another approach to fault analysis is known as fault sampling. This is used in circuits where it is impossible to fault every node in the circuit. Nodes are randomly selected and faulted. The resulting fault-detection rate may be statistically inferred from the number of faults that are detected in the fault set and the size of the set. As with all probabilistic methods it is important that the randomly selected faults be unbiased. Although this approach does not yield a specific level of fault coverage, it will determine whether the fault coverage exceeds a desired level. The level of confidence may be increased by increasing the number of samples.

\subsection{Fault Classifications}

Actual generation of the test set is a small part of the design-for-test process. What a designer is doing up to that point is determining if the design is testable. Thus, test generation is also a process of determining, or debugging, the testability of the design. Part of this process is understanding the types of faults that can and cannot be detected.

Automatic test pattern generation divides faults into various hierarchical categories. These categories are based on how faults were detected or why they could not be detected. The two main categories of faults are untestable and testable. Figure 6 gives a graph of faults classifications.

At the start of the process, all faults start out as undetected. During ATPG, each fault gets assigned to one of the other categories.

Faults that can not be proven untestable are considered testable faults. Testable faults include detected, possible-detected, ATPG untestable, and undetected.

Untestable faults are faults for which no pattern exists to detect them. Untestable faults can not cause any functional failures, and they are therefore excluded when calculating test coverage. Untestable faults include unused, tied, blocked, and redundant 
faults.

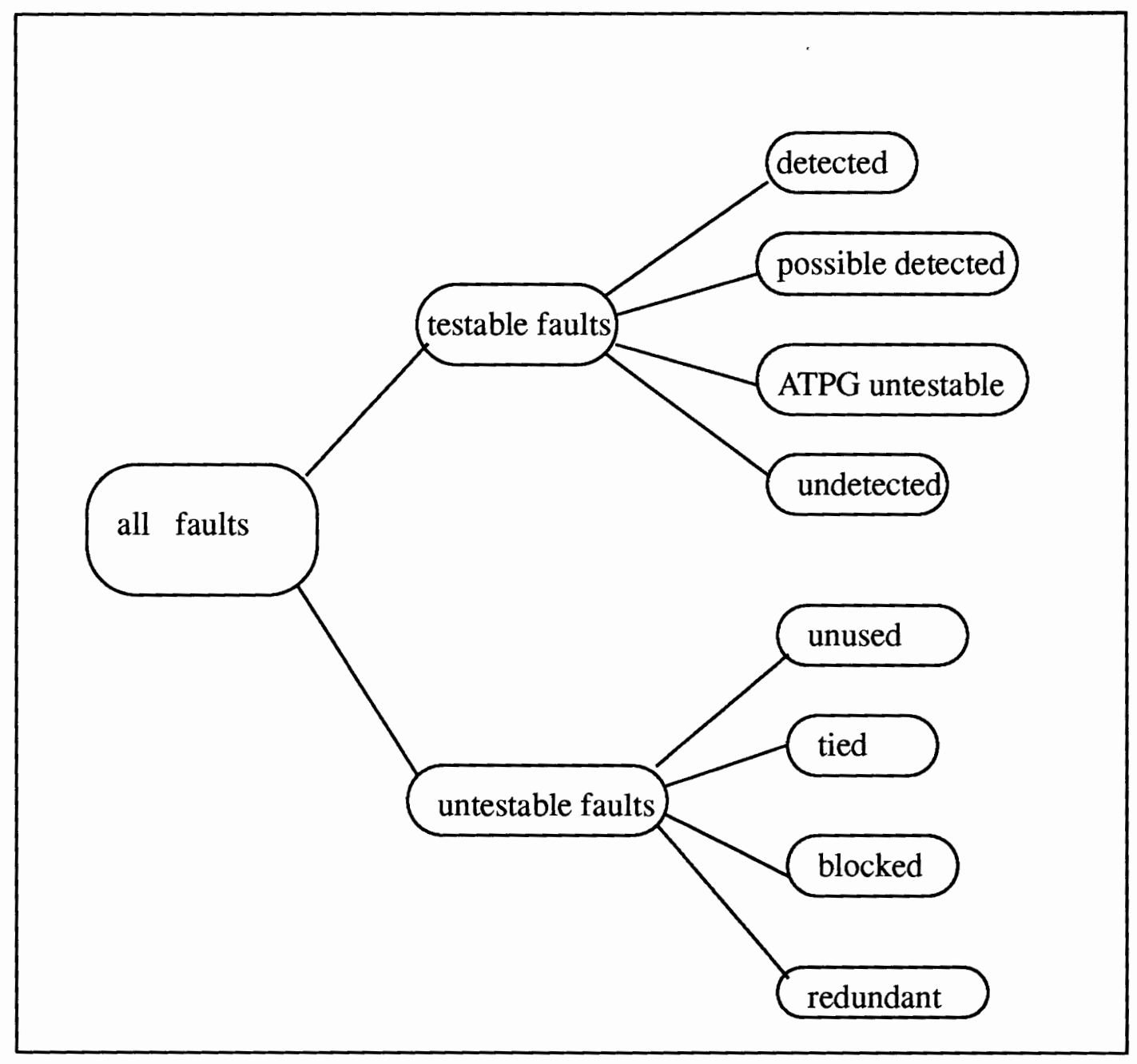

Figure 6: Faults classifications

The understanding of the fault classification is essential to improve the testability of a design. Detailed discussion of these fault classes are given in section 5.3.3. 


\subsection{Testability Measures}

Testability is a design attribute that measures the ability to create a program that comprehensibly tests the quality of a manufactured device. It is a combination of a design's controllability and observability.

The aim of ATPG is to produce test pattern sets that are highly effective for fault detection. The standard measures of this effectiveness include test coverage, fault coverage, and ATPG effectiveness. While each of these measures the quality of the test set, the calculations for each differ slightly.

Test coverage is typically of the highest concern. This is the number that gives the most realistic assessment of the testability of the design. Test coverage measures the percentage of all testable faults that are tested by the test pattern set.

Fault coverage consists of the percentage of all faults that are tested by the test pattern set as well. However, fault coverage treats untestable faults the same as undetected faults. A fault simulator can give this number. If the test coverage and fault coverage vary significantly, there might be a lot of design redundancy or tied logic (nodes in a design are tied to a specific logic value, such as ground or Vcc), or there might be a problem with the netlist.

ATPG effectiveness measures the ability of the ATPG tool to either create a test for a fault or prove that a test cannot be created for a fault. A large difference between test coverage and ATPG effectiveness should be a cause for concern, and could reflect things such as a high number of possible-detected faults, or constraint values on the inputs of a circuit that are hindering ATPG tool's ability to detect faults.

Formulas for test coverage and fault coverage are given as follows:

$$
\begin{aligned}
& \text { test coverage }=[D T+(P D * \text { posdet_credit })] / T E, \\
& \text { fault coverage }=[D T+(P D * \text { posdet_credit })] / F U L L
\end{aligned}
$$

where $D T$ is the number of detected faults, $P D$ is the number of possible detected faults, posdet_credit is the value that specifies how much weight should be given to possible detected faults when making calculations, $T E$ is the number of total testable 
faults which is the sum of detected, possible detected, ATPG untestable, and undetected faults, Full is the number of all faults which is the sum of testable and untestable faults. The formula for ATPG effectiveness is not given here, since there is no standard formula to calculate it, different ATPG tools use different formulas.

These formulas were used in verifying the testability of our cube calculus machine. 


\section{CHAPTER III}

\section{DESIGN STRATEGIES FOR TEST}

Basic test theory is introduced in last chapter. Special design techniques can be used to improve the testability of a design. Testability design strategies, especially, scan design strategy which was used in the testability design of our cube calculus machine are presented in this chapter.

\subsection{Design for Testability}

Traditionally, design and test have been separate processes. And traditionally, the test process has been primarily performed at the end of the design cycle. Test is becoming increasingly costly as the technology gets more advanced. Thus, the testing performed in the traditional manner, at the end of the design cycle, is becoming increasingly more difficult.

However, to ensure that a design is highly testable, designers can employ special techniques at various points in the design phase to improve the testability. This merging of design and test processes to achieve designs with high testability is called designfor-test (DFT).

Design created using DFT techniques have lower testing and test development costs, improved product quality and reliability, and a reduced time to market. Cost, quality, and time to market are the basic constraints of any design project. DFT techniques benefit the design process in the following areas.

In general, as the technology gets more and more advanced, more circuitry can be 
placed onto a smaller area. However, while gate count is increasing -- seemingly without limit -- the number of input and output pins is still tightly constrained. This leads to a decrease in the ratio of $\mathrm{I} / \mathrm{O}$ pins to gates, which makes getting at these gates for testing purposes a more and more difficult endeavor. And more gates per chip generally means a greater likelihood of device failure.

Also, the widely adopted top-down design methodologies create a testing nightmare. By using the high-level hardware description language, and synthesizing, the designer can lose familiarity with his own design. This can make manual test pattern generation impractical.

Efficient testing is critical to the success of a product. It has been shown that the cost of finding defective circuits increases 10 times for each level of assembly. Therefore, the earlier in the process the defects are caught, the less expensive they are to fix. In order to solve this testing crisis, the designer or test engineer must utilize DFT techniques throughout the development process. These DFT techniques include adding special circuitry into a design to aid testability, checking the design to ensure that it is free from design rule violations -- before it gets to the ASIC vendor, and quickly and automatically generating an efficient set of test patterns to test the manufactured device.

Thus, enhanced design testability implemented early in the design process, can generate predictable test results and can greatly reduce test development time and cost. Catching rule violations up front in the design process reduces the costly iterations with the ASIC vendor. And an efficient set of test patterns both reduces costs of testing the finished device and can help pinpoint problems in the manufacturing of the device. These are the benefits we can expect to achieve by employing DFT strategies in the design process.

There are two main approaches to DFT: ad-hoc and structured. 


\subsection{Ad-Hoc Test Techniques}

Ad-hoc test techniques, as their name suggests, are collections of ideas aimed at reducing the combinational explosion of testing. They use design practices to enhance a design's testability without making major changes to the design style. Common adhoc techniques include:

- partitioning large sequential circuits.

- $\quad$ adding test points (control and observation points).

- providing for easy state reset.

- reducing redundant logic.

- reducing asynchronous logic.

- isolating clocks from the logic.

In general, ad-hoc testing techniques represent a bag of tricks developed over the years by designers to avoid the overhead of a systematic approach to testing, which will be described in the next section. While these general approaches are still valid, process densities and chip complexities necessitate a structured approach to testing.

\subsection{Scan Based Test Techniques}

Structured DFT implies a more systematic and automatic approach to greatly enhance the design testability. The goal of structured DFT is to increase the controllability and observability of a circuit. There are various methods for accomplishing this. The most common are the scan design techniques, which change the internal sequential circuitry of the design. 


\subsubsection{Scan Design}

Test generation for combinational designs can process $100 \mathrm{~K}$ gates per hour. Test generation for sequential designs can take much longer. The test generation for sequential designs must go forward and backward in time when it remakes decisions, thus adding another level of complexity to the task.

Scan design, in this case the internal scan, is a structured design-for-test technique that increases the controllability and observability (that is, the testability) of a design. Scan design architectures consist of the serial connection of a design's storage elements into a scan register. The process for inserting scan circuitry replaces sequential elements with scannable sequential elements (scan cells) and then stitches them into a scan register (scan chain). The scan chain is the mechanism for shifting data in and out of the design through the primary inputs and outputs, that is controlled by a scan mode pin. Scan design greatly increases the amount of control points and makes the design appear combinational during test, which makes the problem of test generation simpler.

The main problem with testing a sequential device is that it cannot easily be put into a known state. By shifting a test pattern into the design registers, this initializes or controls the design to a known state. By operating the circuit with this known data and capturing the output, this allows the results to be observed. Thus, the scan circuitry eliminates the problem of testing a sequential device--in effect, making the device look combinational during test.

Figure 7 shows abstractly the sequential devices in a design being stitched together into a chain, and being accessed by a primary input and primary output of the design.

Figure 8 and Figure 9 depict a design, both with and without scan, at a very high level. Typical designs contain both combinational and sequential portions. Combinational portions are most easily tested. Scan circuitry can be added to sequential portion to make it appear combinational during testing.

When scan circuitry is added, to get data into and out of the sequential portion of the circuit, the scan data inputs and outputs are needed. These ports must be primary 
inputs/outputs to the design. Additionally, some sort of signal, either an enable or a clock must enable the operation of the scan circuitry. This will be discussed in more detail later.

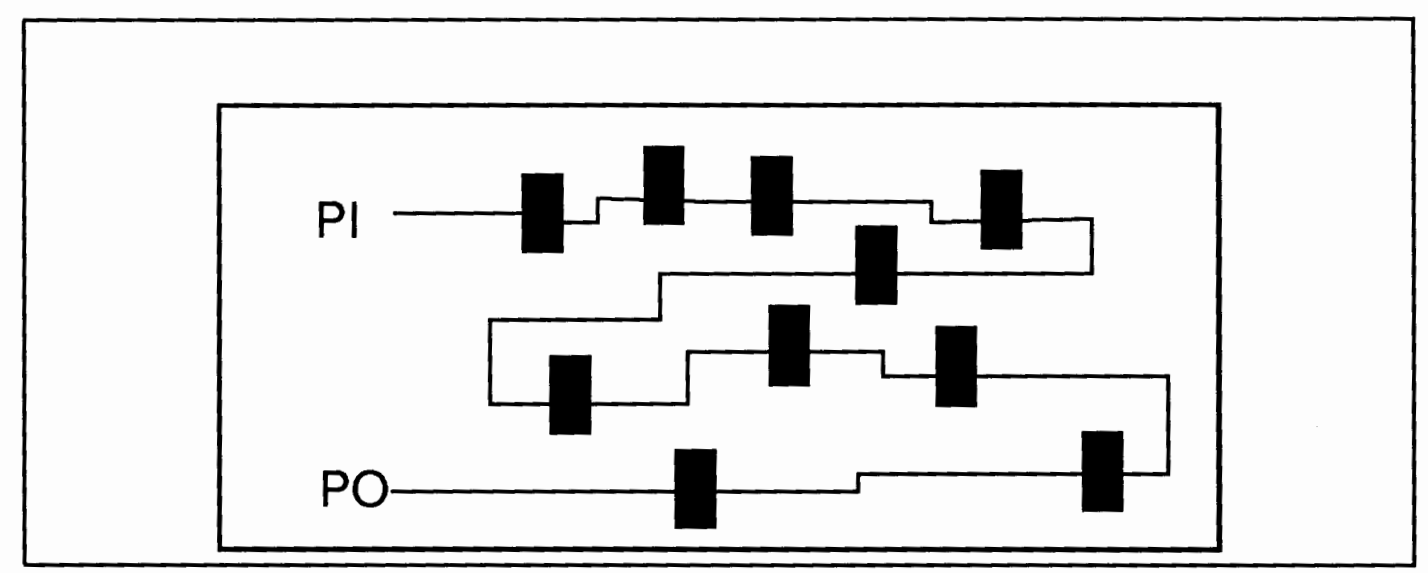

Figure 7: Scan design

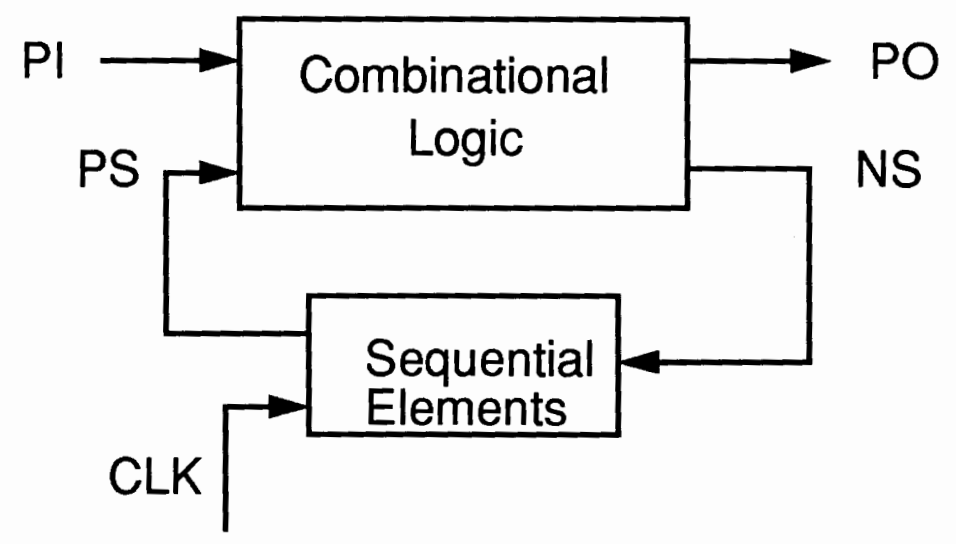

$$
\begin{array}{ll}
\mathrm{PI}=\text { Primary Input } & \mathrm{PO}=\text { Primary Output } \\
\text { NS }=\text { Next State } & \mathrm{PS}=\text { Present State }
\end{array}
$$

Figure 8: Non-scan sequential logic 


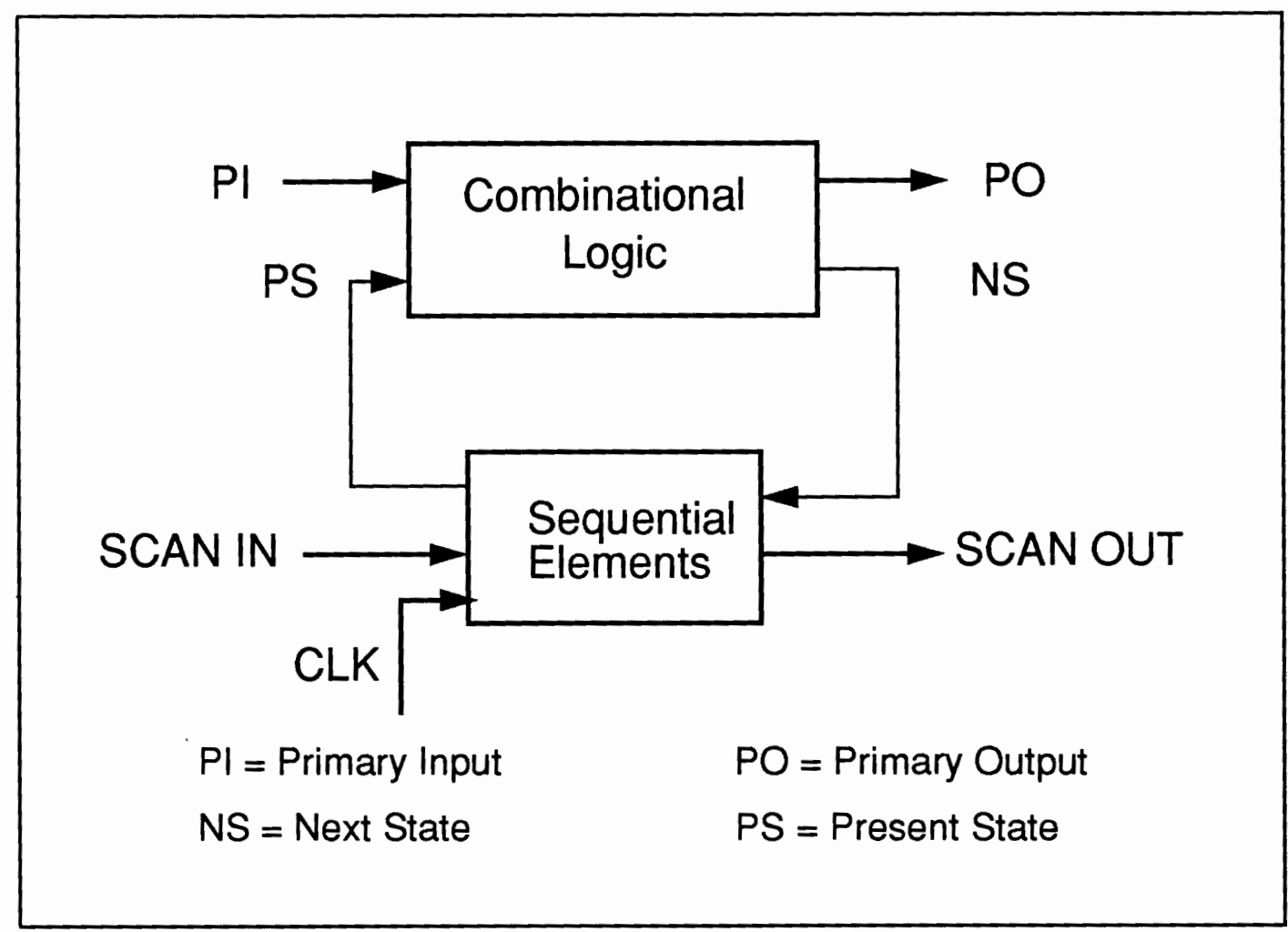

Figure 9: Scan based logic

Figure 10 shows a circuit, at a slightly more detailed level, both before and after scan has been added. Prior to scan, the circuit contains some sequential devices (D flipflops). Inputs to these devices come from both the primary inputs and combinational logic. Outputs from these devices feed the combinational logic.

After scan is inserted, the original flip-flops are replaced with their scannable equivalents. The scannable flip-flops contain additional inputs and outputs for scan data, as well as an input for a scan enable signal. The scan enable signal (sc_en) controls what type of data is allowed into the D flip-flop. When the scan enable is active (during test), the flip-flop is loaded with data from the scan data input (sci). When the scan enable is not active (during regular circuit operation), the data from the regular circuit data input is loaded into the flip-flop. The scan data can be shifted through the flip-flops because the scan data output from each flip-flop feeds the scan data input of the next flip-flop in the chain. 


\section{Before Scan}

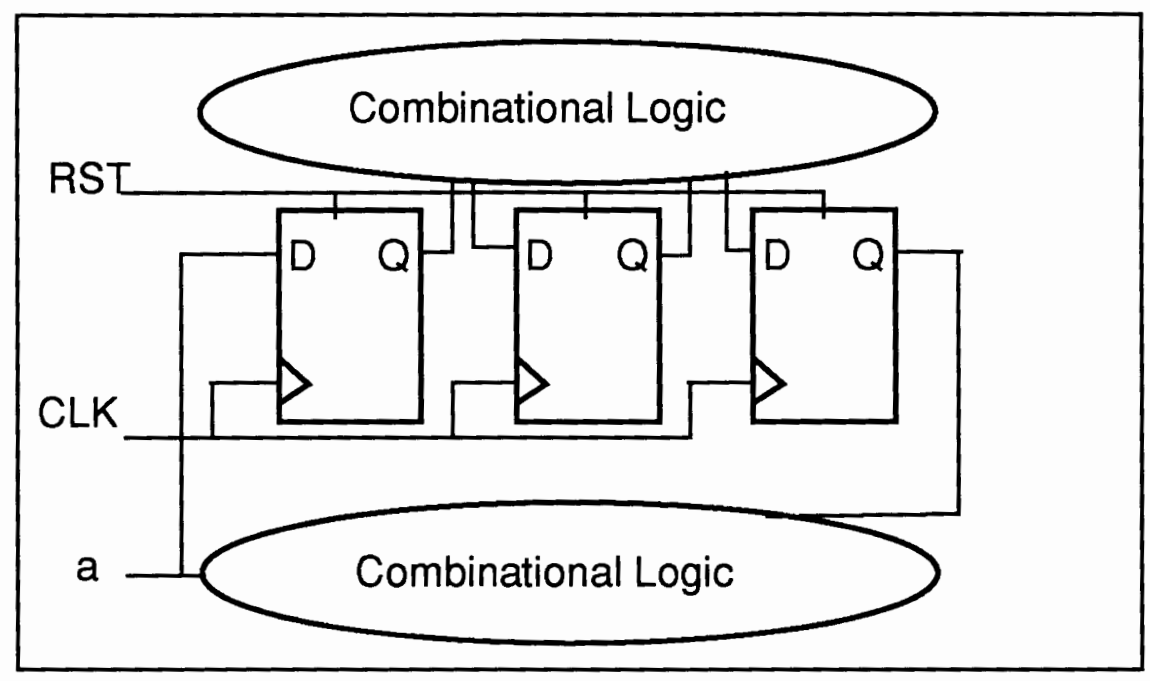

\section{After Scan}

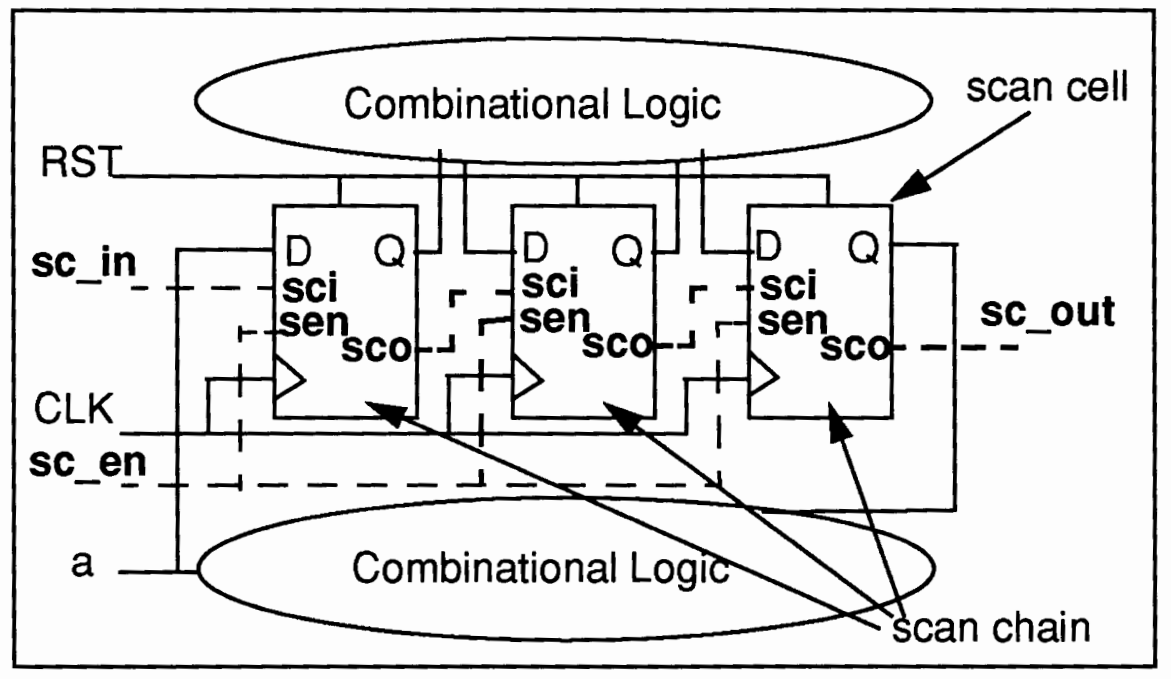

Figure 10: Scan circuit example

In this manner, the scan chain can load the circuit with data (by shifting data into the chain from the sc_in primary input) or can unload data from the circuit (by shifting data out of the scan chain to the sc_out primary output). So not only the control points have been added, but also the observe points, because one can load a known state in, and 
capture, and unload the circuit response through the scan circuitry.

\section{Components of Scan Design}

The elements of a internal scan circuitry include the scan ports, scan clock(s), scan cells, and scan chain(s). The scan ports include scan input (sc_in), scan output (sc_out), and scan enable (sc_en). These elements are shown in Figure 10.

\section{$\underline{\text { Scan Cells }}$}

A scan cell is the basic, independently-accessible unit of the scan circuitry. The connection of a series of scan cells comprises the scan chain. A scan cell contains at least one memory element that lies in the scan chain path. It may contain additional memory elements which may, or may not, lie in the scan chain path.

The memory elements of the scan cells may also have set and/or reset lines, in addition to clock ports.

There are a number of different types of scan architectures. Mainly, they are mux-dff, clocked-scan, and Level Sensitive Scan Design (LSSD).

A mux-dff scan cell contains a single D flip-flop with a multiplexed input line that allows selection of either normal system data or scan data. Figure 11 shows the replacement of an original design flip-flop with scan path circuitry.

In normal operation (scan enable, Sen, is 0 ), system data is passed through the multiplexer to the D input of the flip-flop, and then to the output Q. In scan mode (scan enable, Sen, is 1), scan input data (Sin) is passed to the D input of the flip-flop, and then to the scan output (Sout).

The clocked-scan architecture is very similar to the mux-dff architecture, except instead of using a multiplexer, the circuit uses a dedicated test clock to shift in the scan data. Figure 12 shows the replacement of an original design flip-flop with clocked-scan circuitry. 


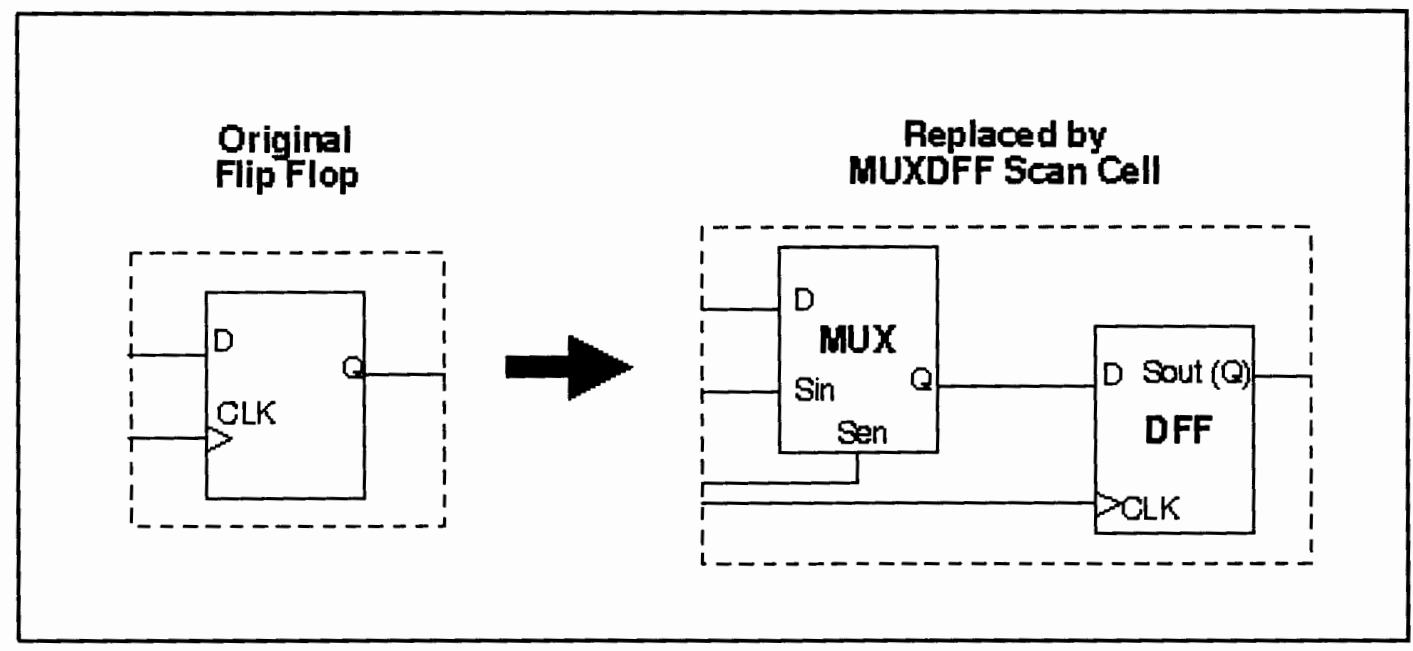

Figure 11: Mux-dff scan replacement

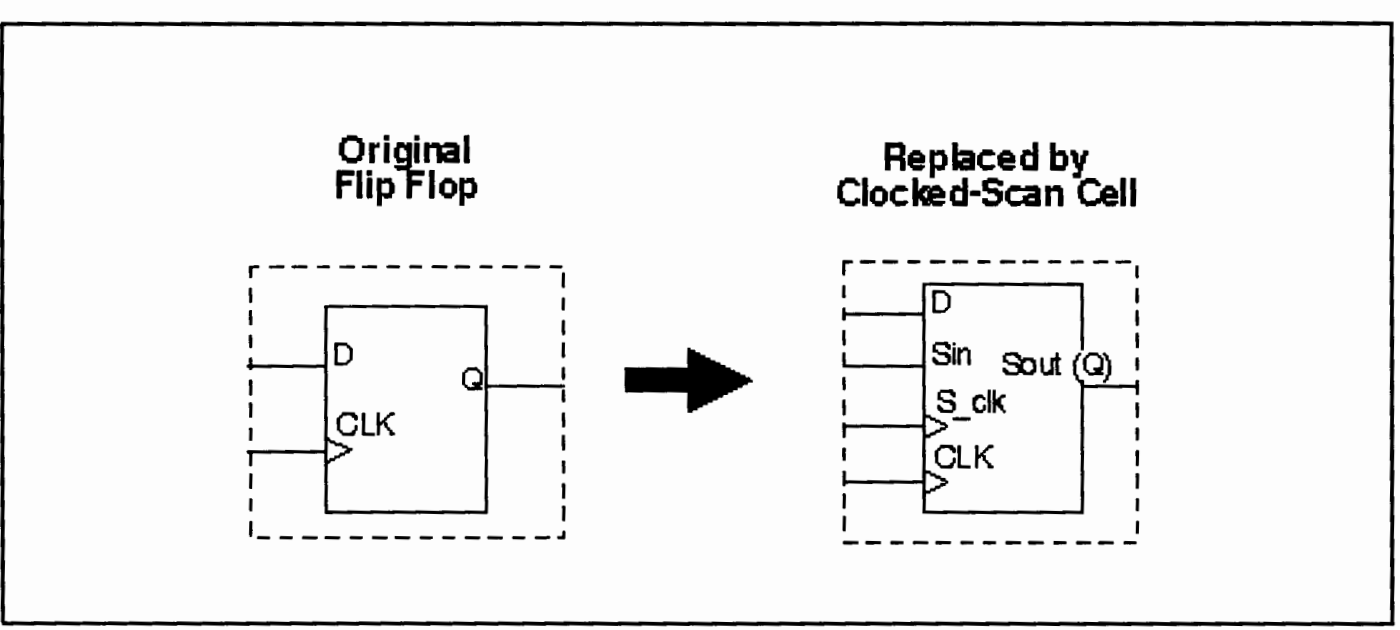

Figure 12: Clocked-scan replacement

In normal operation, the system clock (CLK0 clocks system data (D) into the circuit and to the output $(Q)$. In scan mode, the scan clock (S_clk) clocks scan input data (Sin) into the circuit and to the output (Q).

LSSD, or Level-Sensitive Scan Design, uses three independent clocks to capture data into the two (polarity hold) latches contained within the cell. Figure 13 shows the replacement of an original design latch with LSSD circuitry.

In normal mode, the master latch captures system data (D) using the system clock (CLK) and sends it to the normal system output (Q). In test mode, the two clocks 
(M_clk and S_clk) trigger the operation of shifting test data through both master and slave flip-flop to the scan output (Sout).

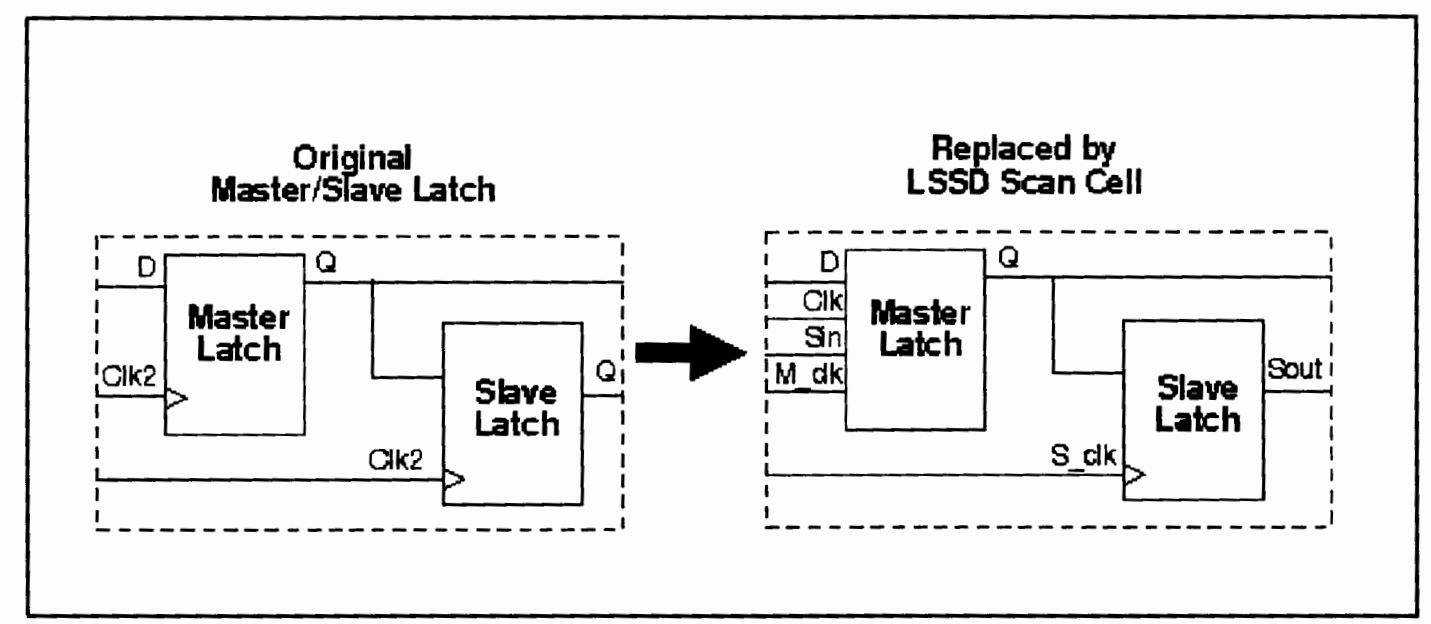

Figure 13: LSSD replacement

\section{Scan Chains}

A scan chain is a set of scan cells serially linked together. A design can contain one or more scan chains. Access to the scan chain is through the scan input and scan input ports. Scan cells are loaded with data by shifting data in through the scan input port. Scan cell data is read by shifting data out through the output port. The length of the scan chain is the number of scan cells in the chain.

As long as the shifting of data between the scan cells can be controlled, the cells can be connected as desired.

The dash lines in Figure 10 connect the three flip-flops to form a scan chain.

\section{Scan Groups}

How does the scan circuitry operate at the chip-level? This question is answered by the notion of scan groups. In a design, there might be two clocks, A and B, each of which clocks scan chain A and scan chain B, respectively. These two chains can often be operated concurrently. However, if two chains share a signal scan_in pin, these 
chains can not be operated in parallel. Regardless of operation, all defined scan chains in a circuit must be associated with a scan group. A scan group is a concept used by Mentor Graphics Design-for-Test tools.

Scan groups are a way to group scan chains based on operation. All scan chains in a group must be able to operate in parallel, which is normal for scan chains in a circuit. However when scan chains cannot operate in parallel, such as in the example above (sharing a common input pin), the operation of each must be specified separately. This means the scan chains must belong to different scan groups.

Test procedure file is used to specify scan chain information for an ATPG tool. Each scan group must have an associated test procedure file. The test procedure file contains instructions, or procedures, for the operation of the scan circuitry of that group during the ATPG process and testing. A typical test procedure contains information about how to load data into and unload data out of the scan cells. Other operations may also be defined in the test procedure file. Test procedure files were used to describe the operation of the scan chain in our cube calculus machine. These examples are given in section 5.3.4.

\section{$\underline{\text { Scan Clocks }}$}

All external pins that are capable of capturing data into the memory elements of the design are considered to be clocks--for scan circuitry purposes. This includes set and reset lines, in addition to traditional clocks. Each clock has an off-state which is the primary input value that results in the clock inputs of the scan elements being at their inactive (off) states for latches, or the initial state of a capturing transition for edgetriggered devices.

In the example shown in Figure 10, both CLK and RST are considered to be scan clocks. The CLK signal is a traditional clock, whose off-state is 0 . CLK is a shift clock. None of the other signals can change the state of the scan cells, so they are not clocks.

Scan clocks are discussed in more detail in Chapter 5 during the testability design of 
the cube calculus machine.

\section{Scan Ports}

The adding of scan circuitry to a design also introduces a number of possible ports to the design. At the most basic level, a scan design needs a port to activate scan test. All other ports, including scan input and scan output ports, can be multiplexed with existing ports. The scan input port allows data to be placed within the scan chain, thus providing controllability of the scan cell states. The scan output port allows data to be extracted from within the scan chain, thus providing observability of the scan cell states.

Depending on the scan style, other ports may be necessary. For example, in MuxDFF style circuitry, a scan enable port is added to determine the type of data allowed into the scan cells. If active, scan data enters the scan cells. If inactive, normal system data enters the scan cells. If the design is a partial-scan, Mux-DFF style, a test clock may be needed to ensure that the contents of non-scan elements are not disturbed.

Often scan ports can be muxed with a normal system ports, so these pins can be used for scan purposes when the design is in test mode. This can eliminate the need for extra pins.

In Figure 10, ports with bold names are scan ports.

\subsubsection{Full Scan Design}

Full Scan is a scan design methodology in which all memory elements in the design are replaced by their scannable equivalents and stitched into scan chains. The concept behind full-scan design is that if the values in all the storage elements of a design can be controlled and observed, the test generation and fault simulation tasks for a sequential circuit can be simplified to that of a combinational circuit.

Figure 14 gives a symbolic representation of a full scan design. The black rectangles represent storage elements that have been converted to scan elements. The line con- 
necting them is the scan path. The rounded boxes represent combinational portions of the circuit.

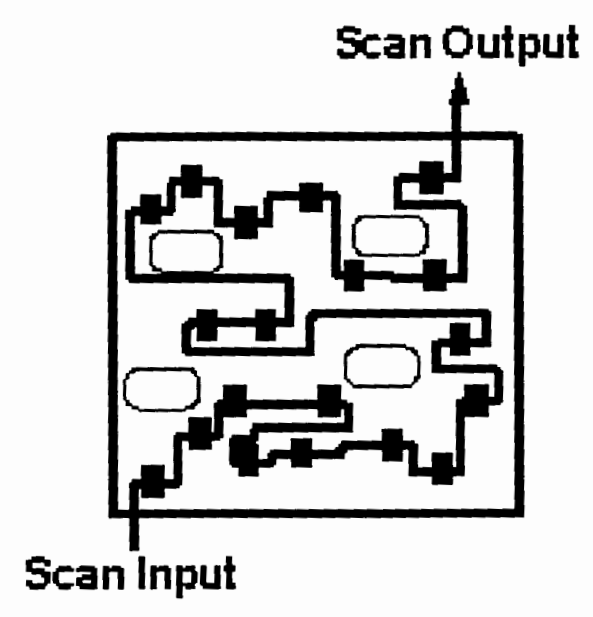

Figure 14: Full scan

The following are the benefits of employing a full scan design:

- Highly automated process. Using scan insertion tools, the process for inserting full scan design is highly automated, thus requiring very little effort from the designer.

- Highly effective, predictable method. Full scan design is a highly effective, well understood, and well accepted method for generating high test coverage.

- Ease of use. In the full scan methodology, the designer, without the aid of a test engineer, can both insert scan circuitry and run ATPG.

- Assured quality. Full scan assures quality because parts containing such circuitry can be tested thoroughly during chip manufacture. If the product is going to be used in market segments that demand high quality, such as aircraft electronics or pacemakers, then full scan technique should be employed. 


\subsubsection{Partial Scan Design}

Partial scan is a design methodology where only a percentage of the storage elements in the design are replaced by their scannable equivalents and stitched into a scan chains. In full scan design, all storage elements are made scannable. However, this may not be acceptable solution for all designs due to area and timing constraints. Using the partial scan method, you can increase the testability of your design with minimal impact on the design's area or timing. In general, the amount of scan required to get an acceptable fault coverage varies from design to design.

Figure 15 gives a symbolic representation of a partial scan design. The rectangles represent sequential elements of the design. The black rectangles are storage elements that have been converted to scan elements. The line connecting them is the scan path. The white rectangles are elements that have not been converted to scan elements and thus, have not been stitched into the scan chain. The rounded boxes represent combinational portions of the circuit.

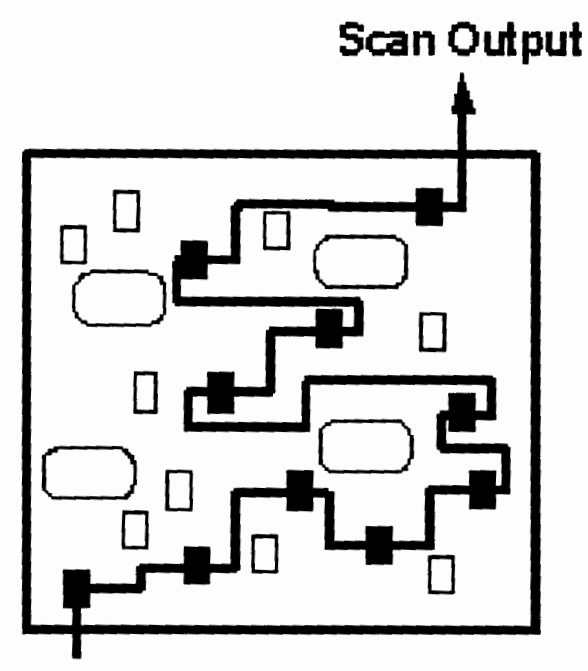

Scan Input

Figure 15: Partial scan 
In the partial scan methodology, the designer, or scan insertion tool must select the desired storage elements for scan chains.

Following are the benefits of employing a partial scan design:

- Reduced impact on area. If we can not tolerate the extra area overhead caused by full scan, we must want to employ partial scan to improve testability to the degree that we can afford.

- Reduced impact on timing. If you can not tolerate the extra delay added to your critical path (due to the added delay of the scan components), you may want to exclude those storage elements from the scan chain. Partial scan gives us the ability to do this.

- More flexibility between overhead and fault coverage. The designer can investigate trade-offs between the area/timing overhead and the acceptable testability improvements.

- Reuse of non-scan macros. If a designer has an existing design block, or macro that he wants to use within the design "as-is", he can include this block within the design. Then he can employ whatever scan method he wants within the rest of the design. This scan strategy implemented in this design would be considered as partial scan.

\subsubsection{ATPG with Full Scan and Partial Scan}

The decision to use either full or partial scan will have a significant impact on the ATPG process. Generally, a full scan approach allows to use a combinational ATPG tool, which requires a minimal test generation effort. Partial scan designs typically use sequential test algorithms, which requires greater test generation effort to produce an acceptable test coverage during ATPG. While combinational ATPG provides a high test coverage in a predictable amount of time, it can be difficult to determine how long it will take for sequential ATPG to produce a desirable test set.

Here is a guideline to help determine whether to use a full scan or a partial scan. Use 
the full scan if its implementation does not push up the package size and does not affect critical timing. Try to start with using full scan unless it breaks some aspect of the design (for instance, the timing on the critical paths, or the die size become unacceptable because of the insertion of full scan.), then justify using partial scan -- not visa versa. Select the best storage elements to convert to scan is very critical in partial scan. 


\section{CHAPTER IV}

\section{A CUBE CALCULUS MACHINE}

As discussed in the introduction, our cube calculus machine is a hardware accelerator for cube calculus operations. In this chapter, an overview of cube calculus operations is given and the architecture of the cube calculus machine is presented.

\subsection{A Review of Cube Calculus}

The main concepts of the cube calculus are those of a cube and an array of cubes. The cube can represent one of the following:

- a product of literals,

- a sum of literals,

- an exclusive sum of literals.

In a binary logic, a literal is a binary variable with or without negation. In a nonbinary (multiple-valued) logic, a literal is a variable with its set of values for which the variable is true.

A multiple-valued input, binary output, incompletely specified switching function $f$ (multiple-valued function, in short) is a mapping

$$
f\left(X_{1}, X_{2}, \ldots, X_{N}\right): P_{1} \times P_{2} \ldots P_{N} \rightarrow B
$$

where $X_{i}$ is a multiple-valued variable, $P_{i}=\left\{0,1, \ldots, p_{i}-1\right\}$ is a set of truth val-

ues that this variable may assume, and $B=\{0,1, X\}$ (" $X$ " denotes a don't care 
value). $N$ here denotes the number of variables (literals, positions).

This is a generalization of an ordinary $N$-input switching function $f: B^{N} \rightarrow B$. For any subset $S_{i} \subseteq P_{i}, X^{S_{i}}$ is a literal of $X_{i}$ representing the function such that

$$
X_{i}^{S_{i}}= \begin{cases}1 & \text { if } X_{i} \in S_{i} \\ 0 & \text { if } X_{i} \notin S_{i}\end{cases}
$$

where: $X^{S_{i}}{ }_{i}$ is a literal, $S_{i}$ is the set of variables for which the literal is true, and $X_{i}$ is the variable.

For example, for a four-valued input logic: $X^{\{0,1,2\}}=1$ if $X \in\{0,1,2\}$, which means, $X^{\{0,1,2\}}=1$ if $X=0$ or $X=1$ or $X=2$. Otherwise $X^{\{0,1,2\}}=0$, which means that for $x=3$ it holds: $X^{\{0,1,2\}}=0$.

A product of literals, $X^{S_{1}}{ }_{1} X^{S_{2}}{ }_{2} \ldots X^{S_{N}}{ }_{n}$, is referred to as a product term (also called term or product, for short). Such term is represented as a cube. A product term that includes literals for all function variables $X_{1}, X_{2}, \ldots, X_{N}$ is called a full term. Any literal of the form $X^{P_{i}}$ is identically equal 1 , since the literal is true for all possible values of $X$. Hence, we often write $X^{P_{i}}{ }_{i} X^{S_{j}}{ }_{j}$ as $X^{S_{j}}{ }_{j}$.

A sum of products is denoted as a Sum Of Product Expression (SOPE) while a product of sums is called a Product Of Sums Expression (POSE). An EXOR of product will be called a Exclusive Sum Of Products form (ESOP). A product of EXORs will be called a Product Of Exclusive Sums expression (POES). SOPE, POSE, ESOP, and POES are all represented as arrays of cubes. Products of SOPEs (PSOPEs) are also used for the Generalized Propositional Formulas form. They are represented as arrays of arrays of cubes.

Switching functions with multiple-valued inputs, binary outputs, find several applications in logic design, pattern recognition, and other areas. In logic design, they are pri- 
marily used for the minimization of PLAs that have decoders on the inputs, minimization of multi-output binary functions and state assignment of Finite State Machines (FSM). A Programmable Logic Array (PLA) with $r$-bit decoders directly realizes a SOPE of $2^{r}$-valued input, two-valued output, function.

Positional notation uses one bit for every possible value of a literal. If the literal is true for a specific value, the corresponding bit is set to a 1. For example, assuming that $X_{1}, X_{2}$, and $X_{3}$ all have 3 possible values, a cube of $X^{01}{ }_{1} X_{2}^{2} X^{012}{ }_{3}$ is equivalent to a cube of $X^{110}{ }_{1} X^{001} X^{111}$ in positional notation. For a cube of $n$ binary variables, $2 n$ bits are needed to represent it in positional notation.

Let's denote by $2 n$ the number of bits of a word (register) that contains a cube in positional notation. To focus our considerations, we assume $2 n=32$ bits. We can have as many as $n=16$ binary variables in a cube. The encoding is as follows: $x-01, \bar{x}-10$, don't care (often denoted by $X$ ) - 11 , and contradiction - 00 . Contradiction means that the literal is not true for any possible value of $x$.

In positional notation, the intersection of two cubes representing products of literals simply corresponds to a bit-by-bit product of the respective words.

For example, assuming four binary variables, $a, b, c$, and $d$, for a cube $A=a b$ and a cube $B=b c \bar{d}$, the product of cube $A$ and cube $B$ is

$$
\begin{aligned}
& a b \cdot b c \bar{d}=a b X X \cdot X b c \bar{d}=\left[\begin{array}{llll}
01 & 01 & 11 & 11
\end{array}\right] \cdot\left[\begin{array}{llll}
1 & 01 & 01 & 10
\end{array}\right]=\left[\begin{array}{llll}
01 & 01 & 01 & 10
\end{array}\right] \\
& =a b c d
\end{aligned}
$$

When two opposite literals are multiplied, if the pair 00 is generated from the bit wise operation, then the resultant cube is a contradiction.

For example, assuming two binary variables, $a$ and $b$, the product of cube $a b$ and cube $a \bar{b}$ is: $a b \cdot a \bar{b}=\left[\begin{array}{ll}01 & 01\end{array}\right] \cdot\left[\begin{array}{ll}01 & 10\end{array}\right]=\left[\begin{array}{ll}01 & 00\end{array}\right]=$ contradiction, which means that the intersection of these two cubes is empty.

For multi-valued input logic, the positional notation uses for each variable as many 
bits as that variable can have values. For instance, four bits are used to represent a 4valued variable. Assuming a variable with 4 values and a second variable with 6 values, the product $X^{A_{1}}{ }_{1} X^{A_{2}}{ }_{2}=X^{0,1,2}{ }_{1} X^{1,3}{ }_{2}$ is represented as a cube

$$
A=\left[A_{1}, A_{2}\right]=\left[\begin{array}{ll}
1110 & 010100
\end{array}\right]
$$

As we can see, in positional notation we consequently enumerate bits representing logical values from left to right, starting with the bit for value 0 .

\subsection{A Review of Cube Calculus Operations}

The cube calculus operations presented in this thesis can be categorized into three groups. They are simple combinational operations, complex combinational operations, and sequential operations. In this section, we will show the mathematical formulas for these operations on multiple-valued input variables. The operations for binary variables can be easily derived from these formulas.

For illustration purpose, we will present first the simple combinational operations, then the sequential operations, and last the complex combinational operations.

\subsubsection{Simple Combinational Operations}

The first group of cube calculus operation is the simple combinational operations. This group includes intersection and supercube, and produce a single resultant cube for each pair of operand cubes

\section{Intersection}

The definition of the intersection operation for cubes $A$ and $B$ in positional notation 
is:

$$
A \cap B=\left\{\begin{array}{cl}
{\left[A_{1} \cap B_{1}, \ldots, A_{N} \cap B_{N}\right]} & \text { if there is no such i that } A_{i} \cap B_{i}=\varnothing \\
\phi & \text { otherwise }
\end{array}\right.
$$

where $A_{i}$ is the $\mathrm{i}$-th literal (position) of cube $\mathrm{A}, A_{i} \cap B_{i}$ is a set intersection of sets $A_{i}$ and $B_{i}$ in positional notation; $\varnothing$ denotes a vector of zeros $(00 \ldots 0)$ with as many bits as variable $\mathrm{i}$ has; $\phi$ is an empty set (a contradiction which may be signalized). The resultant cube is the cube that is included in both $A$ and $B$. An example of intersection operation is shown here:

$$
\begin{gathered}
\text { for cube } A=X^{0,1,2} Y^{1,2,3}=[1110000-0111000] \\
\text { and cube } B=X^{0,1,3,6} Y^{0,2,5}=[1101001-1010010], \\
\qquad A \cap B=X^{0,1} Y^{2}=[1100000-0010000]
\end{gathered}
$$

\section{Supercube}

The supercube of two cubes $A$ and $B$, is the smallest cube that includes all the literals of both $A$ and $B$. The supercube operation of cubes $A$ and $B$ is defined as follows:

$$
A \cup B=\left[A_{1} \cup B_{1}, \ldots, A_{N} \cup B_{N}\right]
$$

where $A_{i} \cup B_{i}$ is set union. An example of supercube operation is shown here:

$$
\begin{gathered}
\text { for cube } A=X^{0,1,2} Y^{1,2,3}=[1110000-0111000] \\
\text { and cube } B=X^{0,1,3,6} Y^{0,2,5}=[1101001-1010010], \\
A \cup B=X^{0,1,2,3,6} Y^{0,1,2,3,5}=[1111001-1111010]
\end{gathered}
$$

\subsubsection{Sequential Cube Calculus Operations}

The second group is the sequential cube calculus operations. The cube calculus operations of this group that we will discuss here are crosslink, non-disjoint sharp, disjoint 
sharp, standard consensus, symmetric consensus, and asymmetric consensus.

Each sequential cube calculus operation can be described by the following pattern using the argument cubes $A$ and $B$ as:

$$
\begin{aligned}
& A=X{ }_{0}^{A_{0}} X^{A_{1}} \ldots X^{A_{N-1}}{ }_{N-1}, \\
& B=X^{B_{0}}{ }_{0} X^{B_{1}}{ }_{1} \ldots X^{B_{N-1}} N-1,
\end{aligned}
$$

where $A_{j}=\left(a_{0}, a_{1}, \ldots, a_{k_{j}-1}\right)$, and for each bit $a_{m}$ from $A_{j}, a_{m} \in\{0,1\}$, Similarly, $B_{j}=\left(b_{0}, b_{1}, \ldots, b_{k_{j}-1}\right), b_{m} \in\{0,1\}$.

Each variable $X_{j}$ can take one of the variables of 0 through $k_{j}-1 . A_{j}$ specifies the set of values of $X_{j}$ for which $X^{A_{j}}{ }_{j}$ is true. Presence of " 1 " in $A_{j}$ in position $m$ (that is, $a_{m}=1$ ) means, that $X{ }^{A_{j}}$ is true whenever $X_{j}=m$. Presence of " 0 " (that is, $a_{m}=0$ ) means that $X^{A_{j}}$ is false for $X_{j}=m$. Each bit of $A_{j}$ can be either a " 0 " or a " 1 " independently of other bits. For binary logic, $X^{00}{ }_{j}$ is false for any value of $X_{j} ; X^{10}{ }_{j}$ is true only for $X_{j}=0 ; X^{01}$ is true only for $X_{j}=1 ; X^{11}{ }_{j}$ is true for both $X_{j}=1$ and $X_{j}=0$.

The array of resultant cubes produced by the sequential cube calculus operation (op) can be described as follows:

$$
\begin{gathered}
A(o p) B= \\
\left\{C_{k} \mid C_{k}=X_{\left.1 \ldots X_{k-1}^{a f t\left(A_{1}, B_{1}\right)}{ }_{1}^{a f t\left(A_{k-1}, B_{k-1}\right)} X_{k-1}^{a c t\left(A_{k}, B_{k}\right)} X^{\text {bef }\left(A_{k+1}, B_{k+1}\right)} \ldots X_{k+1}^{b e f\left(A_{N}, B_{N}\right)}{ }_{N}\right\}}^{N} X^{c_{j}}\right\}, c_{j}=\left(c_{0}, c_{1}, \ldots, c_{k_{i}-1}\right), i \in(1,2, \ldots, m),
\end{gathered}
$$

where if $m$ is the number of pairs literals for which relation ( $r e l$ for short) is true, and $i$ is the number of $i$-th such pair of $A_{k}$ and $B_{k}$, for which the relation $r e l$ is true. 
An important property of functions before (bef for short), active (act for short), and after (aft for short) is that they are bit-wise functions, that is, bit $c_{i}$ of the result of each of these function is dependent only on bits $a_{i}$ and $b_{i}$ of the arguments and therefore the function is defined for cubes of arbitrary size. Values returned by the rel function are determined as an OR or AND combination (depending on the type of the cube calculus operation) of the partial relations for single bits. Each partial relation is determined in bit-wise fashion, that is, it depends only on the values of the corresponding bits of the arguments. Therefore the rel function is also defined for cubes of arbitrary size.

Pairs of literals for which $r e l$ is true are called specific literals. Variables for which literals are specific will be called specific variables, and their corresponding position will be called specific position. As in the above description, the first resultant cube for a sequential cube calculus operation is produced for the first specific literal selected as the active one. Later, the next specific literal is selected as the active one, and the next resultant cube is produced. This procedure is repeated until the last specific literal has been selected as the active one. For a given resultant cube all the literals with numbers less than the number of the specific literal are of the after type, all the literals with numbers greater than the number of the specific literal are of the before type.

\section{Crosslink}

The crosslink operation on cubes $A$ and $B$ creates an array of cubes defined as follows:

$$
\begin{gathered}
A \square B= \\
\left\{X^{B_{1}} \ldots X^{B_{i-1}}{ }_{i-1} X^{A_{i} \cup B_{i}}{ }_{i} X^{A_{i+1}}{ }_{i+1} \ldots X^{A_{N}} \mid \text { for such } \mathrm{i}=1, \ldots \mathrm{N}, \text { that }\left(A_{i} \cap B_{i}=\varnothing\right)\right\}
\end{gathered}
$$

This is basic operation used in minimization of Generalized Reed Muller forms, ESOPs, and other forms based on EXOR gates. The crosslink operation on cubes $A$ and $B$ is a representation of all minterms of the cubes $A$ and $B$ by an EXOR of multiple cubes. All minterms of cube A and B are included in an odd number of cubes, with all 
other minterms included in an even number of cubes, or in no cube at all. Since $1 \oplus 1=0$ and $1 \oplus 1 \oplus 1=1$, this is a valid representation of cubes $A$ and $B$. It can be seen in the above equation that the crosslink operation is not symmetrical, thus $A \square B \neq B \square A$. When the order of the argument cubes is changed, another set of resultant cubes will be generated, but the number of cubes and the size of the cubes will remain same.

The crosslink operation can only be applied to two cubes when: the cubes are specified for the same variables (that is, the literals which are true for all possible values are in the same positions); the two cubes are of the same degree (the degree of a cube is the number of literals in the cube that are not equal to one). The number of resultant cubes is equal to the distance of the two operand cubes.

An example of crosslink operation for binary cubes is given here:

$$
\begin{gathered}
\text { for cube } A=X^{1} Y^{0} Z^{0} Z^{0,1}=[01-10-10-11] \\
\text { and cube } B=X^{0} Y^{1} Z^{1} V^{0,1}=[10-01-01-11] \\
A \square B=\left\{X^{0,1} Y^{0} Z^{0} V^{0,1}, X^{0} Y^{0,1} Z^{0} V^{0,1}, X^{0} Y^{1} Z^{0,1} Z^{0,1}\right\} .
\end{gathered}
$$

The resultant cubes are a chain of cubes from cube $A$ to cube $B$.

\section{Nondisjoint Sharp}

The nondisjoint sharp (sharp) on cubes $A$ and $B$ is defined as follows:

$$
A \# B=\left\{\begin{array}{ccc}
A & \text { when } & A \cap B=\Phi \\
\phi & \text { when } & B \subseteq A \\
A \#_{\text {basic }} B & \text { otherwise }
\end{array}\right.
$$

where $A \#_{\text {basic }} B$ is defined as follows:

$$
\begin{gathered}
A \#_{\text {basic }} B= \\
\left\{X^{A_{1}}{ }_{1} \ldots X^{A_{i-1}{ }_{i-1} X}{ }^{-B_{i} \cap A_{i}}{ }_{i} X^{A_{i+1}}{ }_{i+1} \ldots X^{A_{N}} \mid \text { for such } \mathrm{i}=1, \ldots, \mathrm{N}, \text { that } \neg\left(B_{i} \supseteq A_{i}\right)\right\}
\end{gathered}
$$


This is a basic operation used in minimization of PLAs, tautology, complementation, and all other general purpose logic operations. The nondisjoint sharp of cubes $A$ and $B$ is the set of largest cubes that are included in cube $A$, but not in cube $B$. The result of the nondisjoint sharp is a nonsymmetrical, unique SOPE. By $B_{i} \supseteq A_{i}$ we denote the relation of set inclusion, that is, that set $B_{i}$ includes set $A_{i}$, in positional notation:

$$
\forall j=0, \ldots, p_{i-1},\left[B_{i}^{j} \geq A_{i}^{j}\right] . \text { Formula } \neg\left(B_{i} \supseteq A_{i}\right) \text { is the predicate that is true }
$$

when the relation $B_{i} \supseteq A_{i}$ is not satisfied. By $B \supseteq A$, we denote positional cube inclusion, that is, $B \supseteq A \Leftrightarrow \forall i=1, \ldots, N \quad\left[B_{i} \supseteq A_{i}\right]$.

An example for nondisjoint sharp is shown here:

$$
U^{1,2,3,4} Z^{1,2} \# U^{2,3} Z^{2,3}=\left\{U^{1,4} Z^{1,2}, U^{1,2,3,4} Z^{1}\right\}
$$

where $U$ is a five-value variable and $Z$ is a three-value variable.

\section{Disjoint Sharp}

The disjoint sharp (sharp) on cubes $A$ and $B$ is defined as follows:

$$
A \# d B=\left\{\begin{array}{ccc}
A & \text { when } & A \cap B=\Phi \\
\phi & \text { when } & B \supseteq A \\
A \# d_{\text {basic }} B & \text { otherwise }
\end{array}\right.
$$

where $A \# d_{\text {basic }} B$ is defined as follows:

$$
\begin{gathered}
A \# d_{\text {basic }} B= \\
\left\{X^{A_{1} \cap B_{1} \ldots X}{ }_{1}^{A_{i-1} \cap B_{i-1}} X_{i-1}^{\neg B_{i} \cap A_{i}} X_{i+1}^{A_{i+1}} \ldots X_{N}^{A_{N}} \mid \text { for such } \mathrm{i}=1, \ldots, \mathrm{N}, \text { that } \neg\left(B_{i} \supseteq A_{i}\right)\right\}
\end{gathered}
$$

The disjoint sharp is basically the same function as the nondisjoint sharp with the same resultant set of minterms, but now represented by an SOPE of which the products each cover a separate group of minterms. If the cubes $A$ and $B$ are disjoint, or mutually exclusive, they do not have a common element, that is, $A \cdot B=\phi$. There is more than 
one way to represent the minterms that result from a disjoint sharp by an SOPE, dependent on the order in which the variables or the argument cubes $A$ and $B$ are given.

An example of disjoint sharp for two four-variable binary cubes is shown here:

$$
\mathrm{XXX1} \# d 111 \mathrm{X}=\{0 \mathrm{XX} 1,10 \mathrm{X} 1,1101\}
$$

\section{$\underline{\text { Standard Consensus }}$}

The standard consensus of cubes $A$ and $B$ is the largest minimal cube that covers parts of the adjacent cubes $A$ and $B$. The operation of standard consensus is defined as follows:

$$
A * B=\left\{\begin{array}{cc}
A & \text { when distance }(A, B)=0 \\
\phi & \text { when distance }(A, B)>1 \\
A \text { basic }^{B} B & \text { when distance }(A, B)=1
\end{array}\right.
$$

where $A{ }^{*}$ basic $B$ is defined as follows:

$$
\begin{gathered}
A{ }^{*}{ }_{\text {basic }} B \\
\left\{X^{A_{1} \cap B_{1}}{ }_{1} \ldots X^{\left.A_{i-1} \cap B_{i-1}{ }_{i-1} X^{A_{i} \cup B_{i}}{ }_{i} X^{A_{i+1} \cap B_{i+1}}{ }_{i+1} \ldots X^{A_{N} \cap B_{N}} \mid \text { for } \mathrm{i}=1, \ldots, \mathrm{N}\right\}}\right.
\end{gathered}
$$

\section{Symmetric Consensus}

The symmetric consensus of two cubes A and B, is the largest minimal cube that covers parts of the two adjacent cubes and is included in both of them. The operation of symmetric consensus is as follows:

$$
A * s B=\left\{\begin{array}{cc}
A \cap B & \text { when } \operatorname{distance}(\mathrm{A}, \mathrm{B})=0 \\
\phi & \text { when distance }(\mathrm{A}, \mathrm{B})>1 \\
A *_{S_{\text {basic }} B} B & \text { otherwise }
\end{array}\right.
$$

where $A{ }^{*} s_{\text {basic }} B$ is defined as follows:

$$
\begin{gathered}
A *_{\text {basic }} B= \\
\left\{X^{A_{1} \cap B_{1}}{ }_{1} \ldots X^{A_{i-1} \cap B_{i-1}}{ }_{i-1} X^{A_{i} \cup B_{i}}{ }_{i} X^{A_{i+1} \cap B_{i+1}}{ }_{i+1} \ldots X^{A_{N} \cap B_{N}}{ }_{N} \mid \text { for } \mathrm{i}=1, \ldots \mathrm{N}\right\}
\end{gathered}
$$


An example of symmetric consensus is shown here:

$$
X^{0,1,2,3,4} Y^{0,1} \text { \#s } X^{0,1,2} Y^{2,3}=X^{0,1,2} Y^{0,1,2,3}
$$

where $X$ and $Y$ are both five-value variables.

\section{Asymmetric Consensus}

The asymmetric consensus is similar to the symmetric consensus, but the operation $\neg\left(B_{i} \supseteq A_{i}\right)$ is additionally checked:

$$
\begin{gathered}
A \# S B= \\
\left\{X_{1}^{A_{1} \cap B_{1}} \ldots X_{i-1}^{A_{i-1} \cap B_{i-1}}{ }_{i=1}^{A_{i} \cup B_{i}} X^{A_{i+1} \cap B_{i+1}}{ }_{i+1} \ldots X_{N}^{A_{N} \cap B_{N}} \mid \text { for such } \mathrm{i}=1, \ldots, \mathrm{N}, \text { that } \neg\left(B_{i} \supseteq A_{i}\right)\right\}
\end{gathered}
$$

An example for asymmetric consensus is shown here:

$$
X^{45} Y^{012345} *_{\mathrm{s}} X^{34} Y^{34}=X^{4} Y^{012345}
$$

We observe that the symmetric consensus and the asymmetric consensus both have the same basic formula, but the conditions that need to be satisfied are different. The symmetric consensus uses $A{ }^{*}{ }_{\text {basic }} B$, which does not check any condition. The standard consensus uses the same formula, but only when condition distance $(A, B)=1$ is satisfied. This condition will be called the prerelation. The asymmetric consensus uses an altered version of $A *{ }_{\text {basic }} B$, which applies a set union for every pair of literals for which the relation $\neg\left(B_{i} \supseteq A_{i}\right)$ is satisfied.

For binary logic, the consensus operations have the same resultant cube. For multivalued logic, the result of the asymmetric consensus can be different from the standard consensus. This is the case, when distance $(A, B)=0$ and there is no such $\mathrm{i}$, that $\neg\left(B_{i} \supseteq A_{i}\right)$. In this case $A^{*} B=A B, A *_{s} B=A{ }_{\text {basic }} B$, and $A * a B=\phi$.

It should be recognized in the above formulas that all sequential operations have the same basic structure. Each resultant cube has one specific literal which is the literal $_{i}$. The operations that have to be performed on other literals depend on the position with 
respect to this specific literal. A resultant cube will not exist for all values of $i$, and should be created only when some relations on literal values are satisfied. Generally, the specific literal has to satisfy a certain relation for all given operations. Essential similarities between the definitions of sharp and crosslink can be distinguished in four aspects:

The relation on literals that must be satisfied in position $s$ to create a cube (like $\neg\left(B_{i} \supseteq A_{i}\right)$ for sharp and $A_{i} \cap B_{i}=\varnothing$ for crosslink), each position $i$ for which this relation is satisfied will be called a specific position of cube.

- The operation execute on the literals of position $i$ (like $\neg B_{i} \cap A_{i}$ for sharp and $A_{i} \cup B_{i}$ for crosslink).

- The operation executed on literals of position $i$ (like copying the literals from $A_{i+1}$ $A_{N}$ to the resultant cube in both the above examples).

- The operation executed on literals after the position $i$ (like copying the literals from $A_{1}$ to $A_{i-1}$ for sharp, and from $B_{1}$ to $B_{i-1}$ for crosslink, to the respective positions from 1 to $i-1$ of the resultant cube).

\subsubsection{Complex Combinational Operations}

The third group of the cube calculus operations is the complex combinational operations. One complex combinational operation that will be presented in this thesis is the prime operation.

\section{Prime Operation}

The prime operation is used in ESOP synthesis. The prime operation of cubes $A$ and $B$ is defined as follows:

$$
A^{\prime} B=X^{\operatorname{bef}\left(A_{1}, B_{1}\right)}{ }_{1} \ldots X^{\operatorname{bef}\left(A_{k-1}, B_{k-1}\right)}{ }_{k-1} X^{\text {act }\left(A_{k}, B_{k}\right)} k X_{k+1 \ldots}^{\operatorname{bef}\left(A_{k+1}, B_{k+1}\right)}
$$




$$
X^{\operatorname{bef}\left(A_{l-1}, B_{l-1}\right)}{ }_{l-1} X^{\operatorname{act}\left(A_{l}, B_{l}\right)}{ }_{l} \underbrace{\operatorname{bef}\left(A_{l+1}, B_{l+1}\right)} l+1 \ldots X^{\operatorname{bef}\left(A_{N}, B_{N}\right)}{ }_{N}
$$

where literals with indices $k$ and $l$ are in specific (in this case the same as active) positions. The number of the specific positions is arbitrary, the specific positions are when relation $A_{i} \cap B_{i}=\varnothing$ is satisfied. The values of the other literals are calculated according to the "bef" function. Function "bef" is defined as bef $\left(A_{i}, B_{i}\right)=A_{i}$. The function "aft" is not used here. For example:

$$
\begin{aligned}
& \text { for binary cubes } A=X^{0} Y^{1} Z^{1} V^{1}=[10-01-01-01] \\
& \text { and } B=X^{1} Y^{0} Z^{0} V^{0,1}=[01-10-10-11] \\
& A^{\prime} B=X^{0} Y^{1} Z^{1} V^{0,1}=[10-01-01-11]
\end{aligned}
$$

The prime operation is calculated as if all the specific positions were active at the same time. There is only one output cube for this operation.

\subsubsection{The general programmable patterns}

We have observed the similarities of formulas for the operations in the sequential group. Combinational cube calculus operations are defined as bit-wise functions on the bits of the arguments. There is no need for defining active, before and after positions for combinational cube calculus operations. For consistency of the description, however, one can observe that all the literals in the case of the combinational cube calculus function are of type before. This way the same computational mechanism can be used to calculate both sequential and combinational cube calculus functions. Simple combinational operations are defined as follows:

$$
A\left(o p_{\mathrm{sc}}\right) B=\left\{\left.C_{k}\right|_{k}=X^{\operatorname{bef}\left(A_{1}, B_{1}\right)} 1 \ldots X^{\operatorname{bef}\left(A_{N}, B_{N}\right)}{ }_{N}\right\}
$$

From the above consideration it follows, that each of the "bef", "aft" and "act" functions is actually a function of two single bits. Therefore each of them can be 
described completely by a 2-variable Karnaugh map. Since a 2-variable Karnaugh map has four minterms enumerated $0,1,2$, and 3 , it can be completely specified by the string of four bits corresponding to those minterms. For instance $(0,1,1,1)$ will correspond to minterms of numbers 1,2 , and 3 , which is $\bar{a} b, a \bar{b}$, and $a b$.

To specify completely the "rel" function, one more bit is needed, which serves to determine the method of combining partial relations from single bits (the OR-type relation or the AND-type relation). AND-type means that all bits within a variable must be satisfied to satisfy the relation, while OR-type means that at least one bit must be satisfied within a variable to satisfy the relation.

This way a whole class of sequential cube calculus operations can be described by specifying $4+4+4+1=13$ bits. One more bit can be used to denote that the "prime" function is going to be calculated, and one more bit to distinguish between sequential and combinational operations. In practical implementation a carry signal is used to help combine partial relations for particular bits.

\subsection{The idea of the Cube Calculus Machine (CCM) Architecture}

In the previous sections we illustrated three categories of cube calculus operations according to the type of results. A careful analysis of all cube calculus operations revealed that there are no more such categories. Let us then categorize all three groups in the most comprehensive terms.

In the first category, there is a single cube output, so-called resultant cube, for one or two operand cubes. The resultant cube can be combinationally generated with bitwise logical operations like AND, OR, EXOR, copying the operand, etc. independently of other bits. This category includes supercube and intersection, and we call them simple combinational operations. In this category the operation on two cubes is a concatenation of operations on all positions (respective pairs of literals of the operands). Contra- 
diction is also detected and signalized so that cube with contradiction is not generated at all.

The second category of operations includes the complex combinational operations. For each pair of operand cubes there is a single resultant cube. This cube is a bit-wise logic operation which is, however, conditioned for each literal by some relation/pattern of the input cubes. Prime operation belongs to this category.

The third category has multiple resultant cubes for one or two operand cubes. Examples of these instructions are crosslink, disjoint and non-disjoint sharps, and both multiple-valued consensuses. A literal of the resultant cube is conditioned by the position of this literal relative to other literals and the current specific position. We call these sequential logic operations. Current specific position will be called an active position. In this category the number of the resultant cubes is equal to the number of times that a certain relation is satisfied for a pair of respective literals of the operand cubes, which means, to the number of specific positions. We observe that the active position during the generation of the $i$-th resultant cube is the $i$-th from left among the specific positions of the cube.

General purpose processors execute efficiently in hardware (in elementary instructions) some simple combinational operations, such as the supercube operation (which is implemented by bit-wise OR instruction). They require programs of many instructions to execute other simple combinational operations, complex combinational operations and sequential logic operations. Let us observe that even the cube intersection on a standard computer is slow, because shifted masks are used to detect contradictory cubes. The CCM was designed to accelerate mainly the execution of sequential logic operations. The original concept was to execute in hardware the subroutines that implement those operations: a subroutine call to a sequential operation would be substituted by a CCM instruction. Little modifications would be required to the existing logic synthesis programs and virtually all programs that use sequential logic operations would benefit with the CCM.

The task of the Control Unit (abbreviated by CU in the sequel) of the Cube Calculus 
Machine is to generate signals for sequential cube calculus operations.

To perform sequential cube calculus operations the CU has to provide the Iterative Logic Unit (ILU) with the correct control signals to calculate the solution cubes. The basic sequential cube calculus operations are shown in Table 2.

Table 2: Cube calculus operations

\begin{tabular}{c|c|c|c|c|c}
\hline \multirow{2}{*}{ function } & \multicolumn{2}{|c|}{ relation $($ rel $)$} & \multicolumn{3}{c}{ output operation } \\
\cline { 2 - 6 } & rel & and/or & before(bef) & active(act) & after(aft $)$ \\
\hline intersection & $I$ & and & $A_{i} \cap B_{i}$ & - & - \\
\hline supercube & $I$ & and & $A_{i} \cup B_{i}$ & & - \\
\hline prime & $A_{i} \cap B_{i} \neq \varnothing$ & and & $A_{i}$ & $A_{i} \cup B_{i}$ & - \\
\hline crosslink & $A_{i} \cap B_{i}=\varnothing$ & and & $A_{i}$ & $A_{i} \cup B_{i}$ & $B_{i}$ \\
\hline sharp & $\neg\left(B_{i} \supseteq A_{i}\right)$ & or & $A_{i}$ & $-B_{i} \cap A_{i}$ & $A_{i}$ \\
\hline $\begin{array}{c}\text { disjoint } \\
\text { sharp }\end{array}$ & $\neg\left(B_{i} \supseteq A_{i}\right)$ & or & $A_{i}$ & $\neg B_{i} \cap A_{i}$ & $A_{i} \cap B_{i}$ \\
\hline $\begin{array}{c}\text { symmetric } \\
\text { cons. }\end{array}$ & $I$ & and & $A_{i} \cap B_{i}$ & $A_{i} \cup B_{i}$ & $A_{i} \cap B_{i}$ \\
\hline $\begin{array}{c}\text { asymmetric } \\
\text { cons }\end{array}$ & $\neg\left(B_{i} \supseteq A_{i}\right)$ & or & $A_{i} \cap B_{i}$ & $A_{i} \cup B_{i}$ & $A_{i} \cap B_{i}$ \\
\hline
\end{tabular}

Sharp, disjoint sharp, and asymmetric consensus operations use the OR-type relation $\neg\left(B_{i} \supseteq A_{i}\right)$. This is OR-type relation because it is sufficient to be satisfied in one bit $\left(B_{j}[i]=0, A_{j}[i]=1\right)$ to be satisfied in the entire variable. By $A_{j}[i], j=0,1$, we denote two bits of signal $A[i]$ in IT $[i]$, so $A_{j}[i]=A_{0}[i]{ }^{\circ} A_{1}[i]$, where ${ }^{\circ}$ means concatenation of signals. Here, IT[i] stands for one iterative cell which we will discuss in more detail later. 
The crosslink operation uses AND-type relation, because the relation $A_{i} \cap B_{i}=\varnothing$ is only satisfied if it is satisfied in every bit. The symmetric consensus uses relation " 1 " which is also an AND-type relation. Since relation " 1 " means "always satisfied", it does not really matter what type of relation is used. Since it is global within a variable, AND-type relation is used.

The relations have to be verified, and the output operations have to be executed, bitwise, on bits of ITs. This is illustrated in Table 3 .

Table 3: Cube calculus operations on bits

\begin{tabular}{c|c|c|c|c|c}
\hline \multirow{2}{*}{ function } & \multicolumn{2}{|c|}{ relation(rel) } & \multicolumn{3}{c}{ output operation } \\
\cline { 2 - 6 } & rel & and/or & before $($ bef $)$ & active $($ act $)$ & after $($ aft $)$ \\
\hline intersection & $I$ & and & $A_{j}[i] \cdot B_{j}[i]$ & - & - \\
\hline supercube & $I$ & and & $A_{j}[i]+B_{j}[i]$ & - & - \\
\hline prime & $A_{j}[i] \cdot B_{j}[i]$ & and & $A_{j}[i]$ & $A_{j}[i]+B_{j}[i]$ & - \\
\hline crosslink & $\neg A_{j}[i]+\neg B_{j}[i]$ & and & $A_{j}[i]$ & $A_{j}[i]+B_{j}[i]$ & $B_{j}[i]$ \\
\hline sharp & $A_{j}[i] \cdot \neg B_{j}[i]$ & or & $A_{j}[i]$ & $A_{j}[i] \cdot \neg B_{j}[i]$ & $A_{j}[i]$ \\
\hline $\begin{array}{c}\text { disjoint } \\
\text { sharp }\end{array}$ & $A_{j}[i] \cdot \neg B_{j}[i]$ & or & $A_{j}[i]$ & $A_{j}[i] \cdot \neg B_{j}[i]$ & $A_{j}[i] \cdot B_{j}[i]$ \\
\hline $\begin{array}{c}\text { symmetric } \\
\text { cons. }\end{array}$ & 1 & and & $A_{j}[i] \cdot B_{j}[i]$ & $A_{j}[i]+B_{j}[i]$ & $A_{j}[i] \cdot B_{j}[i]$ \\
\hline $\begin{array}{c}\text { asymmetric } \\
\text { cons }\end{array}$ & $A_{j}[i] \cdot \neg B_{j}[i]$ & or & $A_{j}[i] \cdot B_{j}[i]$ & $A_{j}[i]+B_{j}[i]$ & $A_{j}[i] \cdot B_{j}[i]$ \\
\hline
\end{tabular}

4.4 The Concepts of Ring of Processors and Interlock Mechanism

The architecture of CCM results from an attempt to optimize the execution of sequential operations, like crosslink and sharp, or consensus and asymmetric consen- 
sus. The CCM architecture directly implements equations of the above style.

All the known software subroutines process the literals sequentially, but for most of the literals the resultant cubes generated will have contradictions and will have to be removed later. We created a completely new architecture to take advantage of the peculiarities of the sequential cube calculus operations [KIDA 91]. The architecture is an iterative logic array (ILU) with iterative signals running from left to right and from right to left of the iterative circuit of Position State Machines (PSMs). The fundamental advantage of this approach is that only cubes without contradictions are generated.

The ILU recognizes the next specific (active) position and generates a resultant cube in each cycle. It realizes, using internal distributed control, the lowest level iterative loop. Therefore, ILU does not need the control unit to execute the basic cube operations: while generating the resultant cube the role of $\mathrm{CU}$ is limited to generating signals REQUEST and checking signals NEXT. ILU is controlled by two types of signals, iterative signals and global signals. Two of those signals, global REQUEST and iterative NEXT work in an "interlock mechanism" that substitutes the "clock" of synchronous machines with a "two-phase non overlapping rippling waveform" of iterative PSMs.

This is a general concept in computer architecture. It consists in taking the control of the lowest level loop off the control unit and putting it directly to the data path, in a distributed form. Figure 16a shows the classical realization of the computer with Control Unit (CU) controlling the Data Path (DP) with instructions and receiving from DP predicates informing about state of DP. Figure 16b shows our approach. There is a sequence of Finite State Machines (FSMs) which communicate among themselves, each with its left and right neighbors. The CU sends controlling instructions, like in the classical case, but it also initializes instructions executed by the sequence of FSMs. It receives the predicates, but also the termination signals from the string of FSMs. CU can be thus treated as one of the ring of FSMs. Our concept can be further generalized as in Figure 16c. Each processor from the string is not an FSM but some simple general-purpose processor from a local CU (FSM) and a local data path. This is a general purpose hierarchical organization of the computer, where the lowest level of control is 
distributed and asynchronous in the ring of processors.

a)

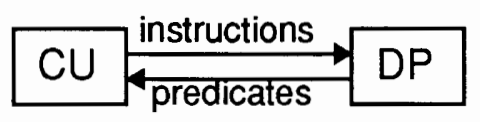

b)

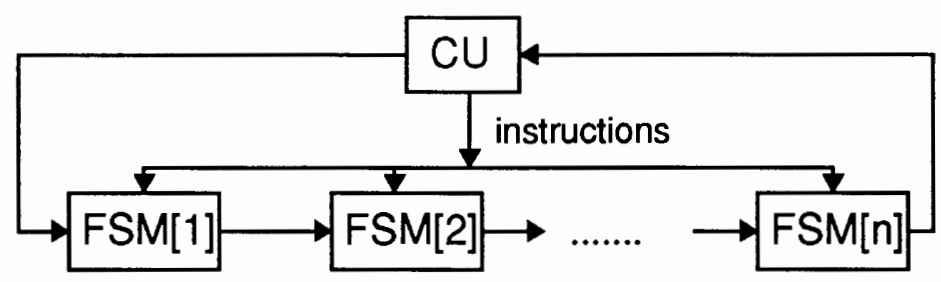

c)

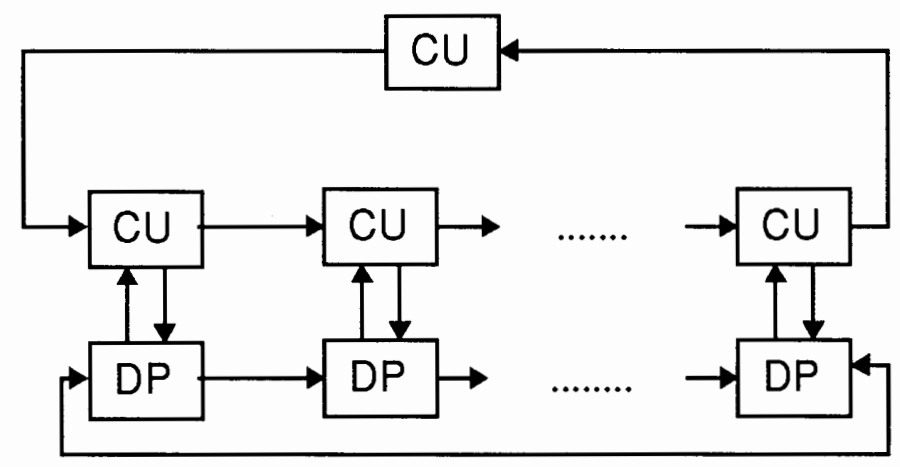

Figure 16: The three architectural models of a general-purpose computer

An analogy that helps to understand the advantages of this architecture over the sequential processing of all literals is to imagine each literal as a domino tile. The linear iterative array has all dominoes lined up in a way that if the first one falls, all next will fall in sequence. For the specific positions the correspondent domino tile is removed. This way, when the control unit pushes the first domino, the domino tiles will fall in a "domino effect" until they reach the gap left by the specific position, where the domino 
effect will stop. At that point the literal is processed and an output cube is generated with each of its literals being a function of its position in the array. If its domino has already fallen down it corresponds to state after, if the literal is being processed, it corresponds to state active, when its domino is still standing, it corresponds to state before. The control unit begins the cycle again by pushing the first remaining domino until there are no more standing dominoes left. This is of course only a general analogy, since dominoes fall one after another, and in our design a group of "dominoes" is "prepared to fall" and next all of them fall at once.

In fact the iterative circuit has a ring configuration and the control unit serves as the first and last domino. This way, it is simple for the control unit to observe the fact that all literals have been processed, without the need to keep track of which literal is being processed and how many remain to be processed.

A co-processor that processes a literal at a time would push a single domino for each cycle and try to improve performance by increasing the rate at which it processes each literal. Among the disadvantages of such a method is that it generates cubes with contradiction that have to be removed. If a circuit to recognize and remove cubes with contradiction were integrated to the architecture, then the rate of generation of resultant cubes would be irregular. The rate of generation of resultant cubes in our architecture is regular, making it suitable for pipelining and systolic processing, which allows to build large parallel structures from them and was one of the main objectives of our approach.

The control unit is a relatively simple Finite State Machine and great part of the control task is distributed in the ILU itself.

\subsection{The Block Level Description of the Architecture}

The CCM chip consists of a processing unit, an interface controller, a register file and a control unit. Figure 17 shows the block diagram of CCM architecture.

The CCM works on cubes in positional representation. It handles multi-valued vari- 
ables of even number of values. Because each IT can calculate two resultant bits, 2 bits of separate literals cannot be stored in the same IT.

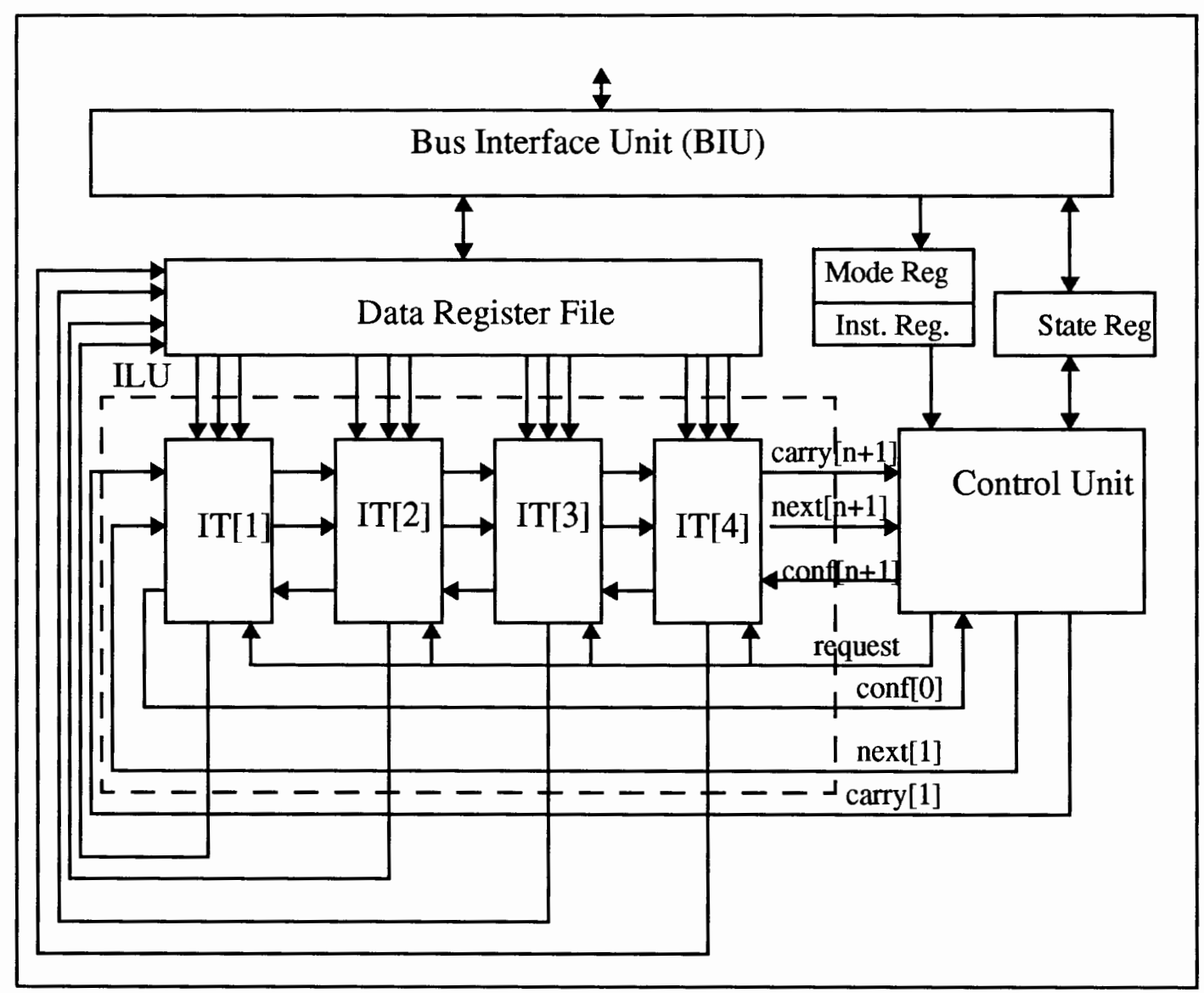

Figure 17: The block diagram of the CCM processor architecture

\section{The Processing Unit}

The processing unit is implemented as an iterative logic array of basic building blocks. It is called Iterative Logic Array (ILU). Single cell (block) from ILU is called ITerative Cell (IT). Blocks IT will be enumerated from left to right: IT[1], IT[2], ...IT[i], ..., IT[n]. (The number of ITs is denoted by $n$, so that the number of bits is $2 n$ and we can process $\mathrm{n}$ binary variables). Each IT[i] includes besides combinational logic a Position State Machine (PSM) that influences the local interpretation of the micro-instructions. In this sense each IT is a small processing unit that processes a part 
of a cube in parallel and communicates with other processors that are connected in a linear organization.

\section{The Iterative Cell}

The IT is the basic building block of the ILU. It processes two bits that can be either a binary variable or two bits (values) of a multi-valued variable.

For explanation purposes we will divide each IT[i] into three blocks according to the function that they perform: IDENTIFY[i], STATE[i] (name of the PSM block) and SIGNALIZE[i]. Figure 18 shows these blocks.

Block IDENTIFY[i] has the task of identifying the position of the IT within the literal and generating a boolean signal VARIABLE[i] that is true when the IT[i] is a part of a literal that complies to the selected relation. This relation, "rel", is encoded in the field of the micro-instruction, REL. Examples of relations are: $A_{i} \cap B_{i}=\varnothing, \neg\left(B_{i} \supseteq A_{i}\right)$, etc. The type of relation verified in ITs is the same for all ITs, because the REL[1:4] is a global controlling signal. To calculate the value of VARIABLE[i], the block IDENTIFY[i] uses two iterative signals, CARRY[i] and CONF[i]. CARRY[i] is an iterative signal that runs from left to right and is true when all ITs of the same literal at the left of the IT satisfy the AND-type relation encoded in rel. CONF[i] (confirm) says that within this position all ITs have satisfied relation. As we see, the signal CARRY goes from left to right, up to the end of variable, and next returns as signal CONF, back to all ITs of this variable. This explanation is only for AND-type relations. The explanation for ORtype relations, and a general explanation will be given in following sections.

Block STATE[i] is an finite state machine essential to execute sequential operations. It is the state of the STATE[i] that stores the position of the IT[i] in relation to the active literal. The STATE[i] is in the state active if the IT[i] is a part of an active literal; it is in the before state if the active literal is to the right of the IT; and in after state if the IT[i] is to the left of the active literal. STATE[i] are initialized to the state before with the global signal CLEAR from CU. 


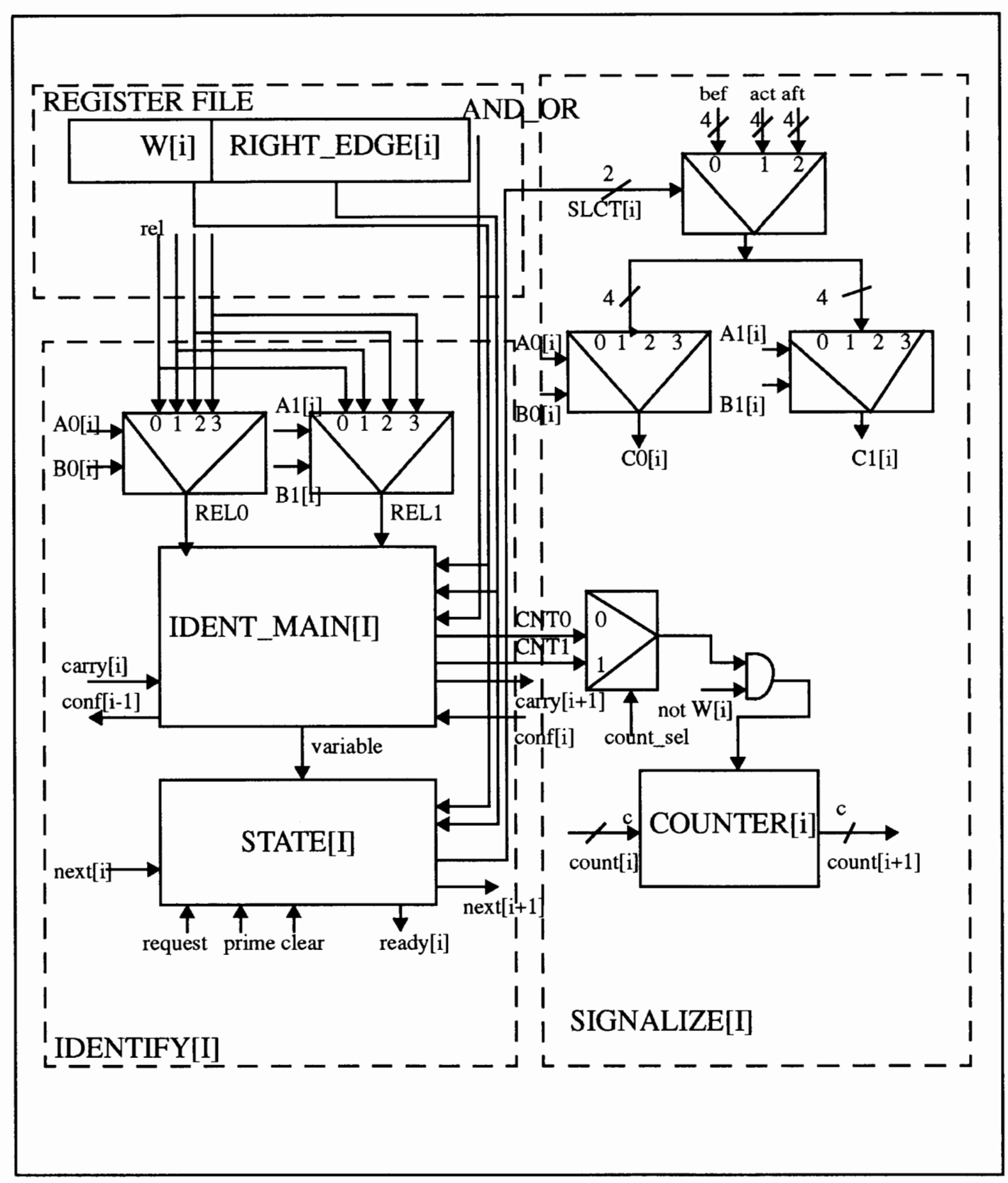

Figure 18: The signals in IT

\section{The Control Unit (CU) and the Bus Interface Unit (BIU)}

The Control Unit (CU) of the CCM receives a code of a high level cube calculus operation (CCM instruction) in the Instruction Register (IR) and translates it into simpler basic operations implemented in the processing unit. 
Bus Interface Unit (BIU) handles the communication between the host computer (or a distributed system of CCMs and controllers) and the CCM. The communication between the BIU and the ILU is done through shared registers/memory, as is the communication between the BIU and the CU. The interfaces between the BIU and the ILU and the $\mathrm{CU}$ were made independent, asynchronous and through a protocol to let the design of the ILU and CU be independent of the BIU. The same CU and ILU can be integrated to different systems by redesigning the interface (BIU) only, and the basic design of the interface for one particular system can be used over and over for different versions of ILU and CU.

\section{The Data Register File}

The shared data register file accounts for storing the input cube, the output cubes, and the intermediate results. Usage of this file prevents also a loss of performance due to the differences in the processing rates of the system and the ILU to feed the input operand cubes, generate the resultant cubes, and transfer them out of the CCM.

There are other registers used for communication and control, among them: the Right Edge Register, the Status Register, the Instruction Register, and the Water Register.

The Status Register stores information useful to support programs in the host processor: the distance of cubes transmitted to the ILU, the number of resultant cubes, flags and semaphores to signalize when the resultant cube is ready or when there is no result.

The Instruction Register controls additionally the data transfer and the iterative signals of the first and the last iterative cells of the ILU.

The Right Edge Register specifies the number of iterative cells (IT) used by each literal of the cube. There is one bit for each IT of the ILU. For example, when the content of Right Edge Register is 010111, the first 2 bit represent a 4-valued variable and will use the first 2 ITs, the second 2 bits are used by another 4-valued variable and will take another two ITs, the third and the fourth variables are 2-valued (binary) and will use only one iterative cell each. When RIGHT_EDGE[i]=1, then IT[i+1] is the beginning of the next literal. 
The Water Register is used to indicate which ITs are not used in the current operation. Since the number of bits required to represent all literals in the argument cubes could be less than the number provided by all ITs, some of the ITs are not used. Water Register $\mathrm{W}[\mathrm{i}]=1$ means the $i$-th IT is not used in the current operation.

\section{Timing Description of the Collaboration of the ILU and the CU}

This section will give more detailed description of the timing of the signals between the ILU and the CU during the execution of a sequential operation.

Figure 19 illustrates the execution of a sequential operation, with the stable state of the ITs of the literals (represented as boxes) after the propagation of the signals showed on the left column.

Sequential operation begins by loading the operand cubes to let blocks IDENTIFY[i] to recognize all the specific positions. The CU keeps the global signals REQUEST $=0$ and CLEAR $=0$, as well as the initial signal NEXT[0] $=0$ and lets all signals VARI$A B L E[i]$ in ITs reach their final values. The interval of time before $C U$ is ready to do something next has to be long enough for the ITs to have their VARIABLE[i] signal stabilized (delay of 1 IT for a binary variable, 2 IT for a 4-valued variable,...). This is represented in Figure 19a. At the end of this phase all specific positions are marked as values 1 of VARIABLE[i] in the corresponding ITs. The $\mathrm{CU}$ can then read the number of resultant cubes from the value of the iterative count signal COUNT from the last IT.

The CU resets the ILU to its initial condition by asserting CLEAR (shown in Figure 19b). By bef we denote the state before (which stands for before active) of STATE. The control unit does not have the control over signals VARIABLE[i] but since A[i], B[i], AND_OR, and the RIGHT_ED[i] remain stable, VARIABLE[i] will remain stable as well. When all IT[i] cells are reset, the CU deasserts signal CLEAR (shown in Figure19c).

The execution of the instruction really begins by the assertion of NEXT for the leftmost IT, NEXT[0], to true. The first literal that has VARIABLE[i] = 1 will become active at the active edge of the REQUEST signal. (If many ITs are used to represent a 
literal, all of them will have VARIABLE[i] = 1, and all will become active. Remember, however, that there is a signal RIGHT_EDGE[i] that is used to distinguish among neighbor variables). Either the first literal becomes active (Figure 19d.1) or signal NEXT may propagate through one or more literals (Figure 19d.2). By act we denote the state active of STATE. By aft we denote the state after (after active) of the STATE. After short time, with stable states of STATE, the CU transmits a resultant cube to output. The CU samples the iterative signal NEXT[i] from the last IT (NEXT[n+1]), if it is true there are no resultant cubes to generate and the operation is finished, if it is false, it means that the signal NEXT[i] has been stopped by a specific position and the CU has to output the resultant cube. If NEXT[n+1] $=0$, the CU makes the active edge of REQUEST signal to prepare for the next cycle. This will make the ITs in the active position to transit to the after state and let the NEXT[i] signal pass to the cells after it (Figure 19e,f). A new cycle can begin for those literals in state before after the cell that changed from active state to after in Figure 19d.1 and Figure 19d.2. Such process is iterated until signal NEXT[i] = 1 passes the entire ILU and, as NEXT[ $n+1]$, reaches the $\mathrm{CU}$; at this point the operation is finished.

4.6 The Detailed Description of the Implementation of the Iterative Cell (IT)

\subsubsection{Iterative Signal in the Block Structure of IT Cells}

The IT cell will be described in full detail in this section.

The transitions of PSMs in blocks STATE[i] are controlled by the global signal REQUEST from the CU and the iterative signal NEXT[i]. Signal REQUEST is just a clock of PSMs (not to be confused with the global clock of CCM). All changes of states of PSMs are under control of REQUEST. 


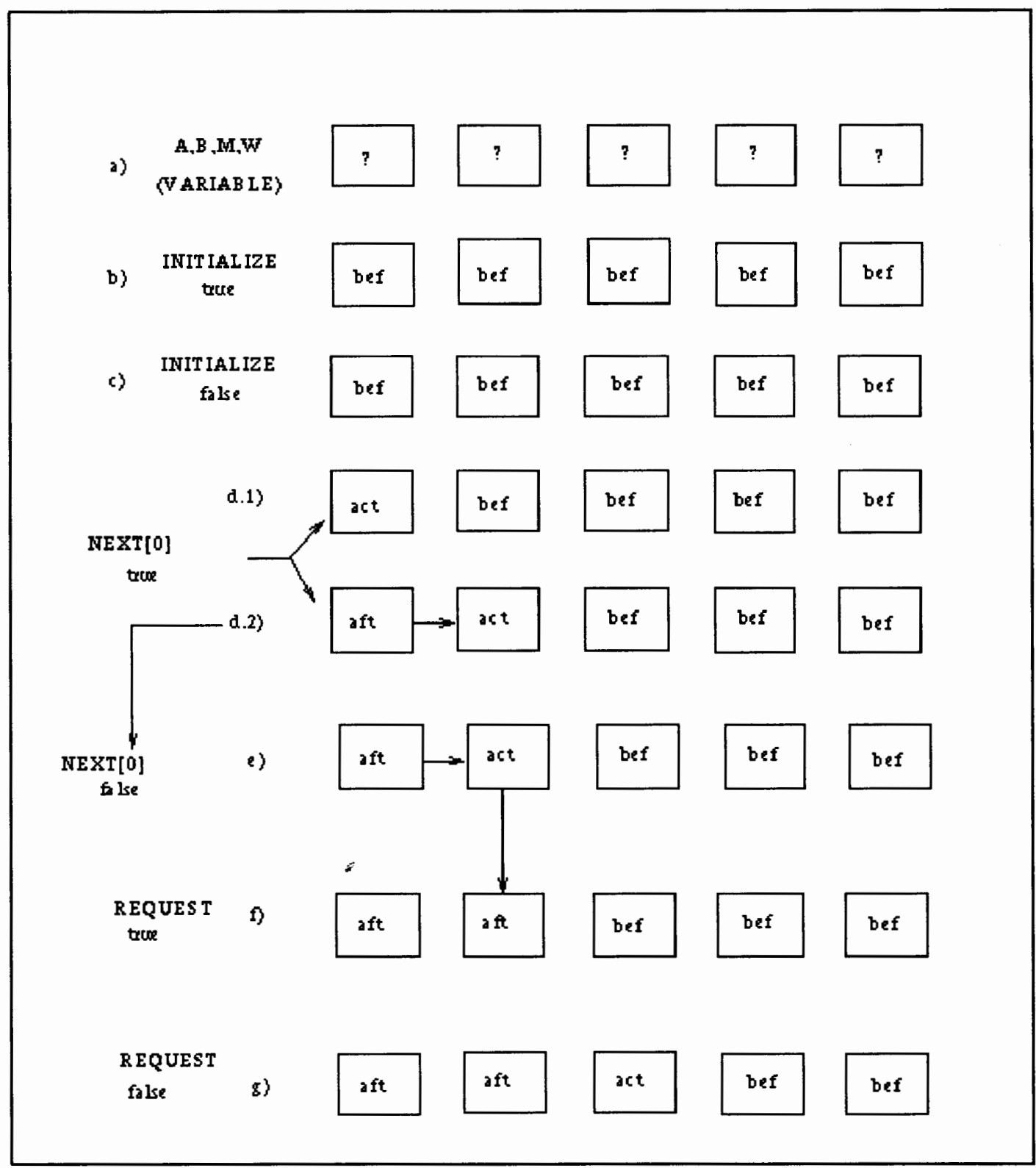

Figure 19: An example of a sequential operation

Since the CU emulates also the IT[0], it has the total control over the ILU.

Since the signal REQUEST is the clock of flip-flops in all PSMs STATE[i], those machine cannot change their internal states other than through REQUEST. The changes of ITs are from state before to states active or after. The detailed mechanism of those changes will be explained in section 4.6.4. 
The signal NEXT[i] has effect only on the IT[i]s that are in the state before. An IT[i] in the state before that receives NEXT[i] = 1 will change to the state after and assert NEXT $[i+1]=1$ at the active edge of the REQUEST signal, if IT[i] is not in a specific position. It will change to state active and pass NEXT[i+1] $=0$ if it is in a specific position.

For sequential operations, the block SIGNALIZE[i] of IT[i] generates a part of the output according to the micro-instruction field S[1:16] and the state of the STATE[i]. The field S[1:16] is taken from some register, and denotes fields rel, bef, act and aft together. (Remember, $4+4+4+4=16$ ). It uses the S[1:16] field and VARIABLE[i] for complex combinational operations, and the $S[1: 16]$ field and the inputs for simple combinational operations.

Block SIGNALIZE[i] has an iterative signal COUNT[1:c] that counts the number of variables (literals) that have the signal VARIABLE[i] $=1$.

Figure 20 shows the block diagram of three ITs in a row, IT[i-1], IT[i], and IT[i+1] of the ILU. The number near a bus means the number of wires in that bus. For instance, number 2 near bus $A[i]$ means there are two wires in this bus, in this case they are $A_{0}[i]$ and $A_{1}[i]$. The number $c$ near the bus COUNT[i] means bus COUNT[i] has $c$ wires. Where the number $c$ depends on how many ITs in the ILU. Since the bus COUNT[i] is used to count how many ITs in the ILU are in the specific position.

Since the REL, BEF, ACT and AFT functions are bitwise, the design of the data-path of CCM reflects this fact. However, since 2 is the smallest number of bits in a literal (binary literal) the IT performs CC operations for 2 bits. In the case of literals of more than two values, several ITs are assigned to such a literal (if number of values of a literal is odd, one of the bits is not used).

There are two signals providing communication between the ITs assigned to the given variable. The CARRY[i] signal provides the means of combining the results of partial relations (computed in each IT). The CARRY[i] signal is propagated from left to right within a literal. In the case of AND-type relation the decision whether the relation is fulfilled or not is made in the last IT in the chain (on the right edge). Then the 
CONF[i] signal is sent back (from the right to the left) to notify all the ITs assigned to the variable that the relation is true. In the case of OR-type relation the decision is made anywhere in the chain - in the first place where partial relation is true. Then the CONF[i] signal is sent immediately back to inform the rest of the ITs about the relation fulfillment; besides that CARRY[i] is propagated all the way to the right edge of the variable in order to produce CONF[i] signal for the remaining ITs.

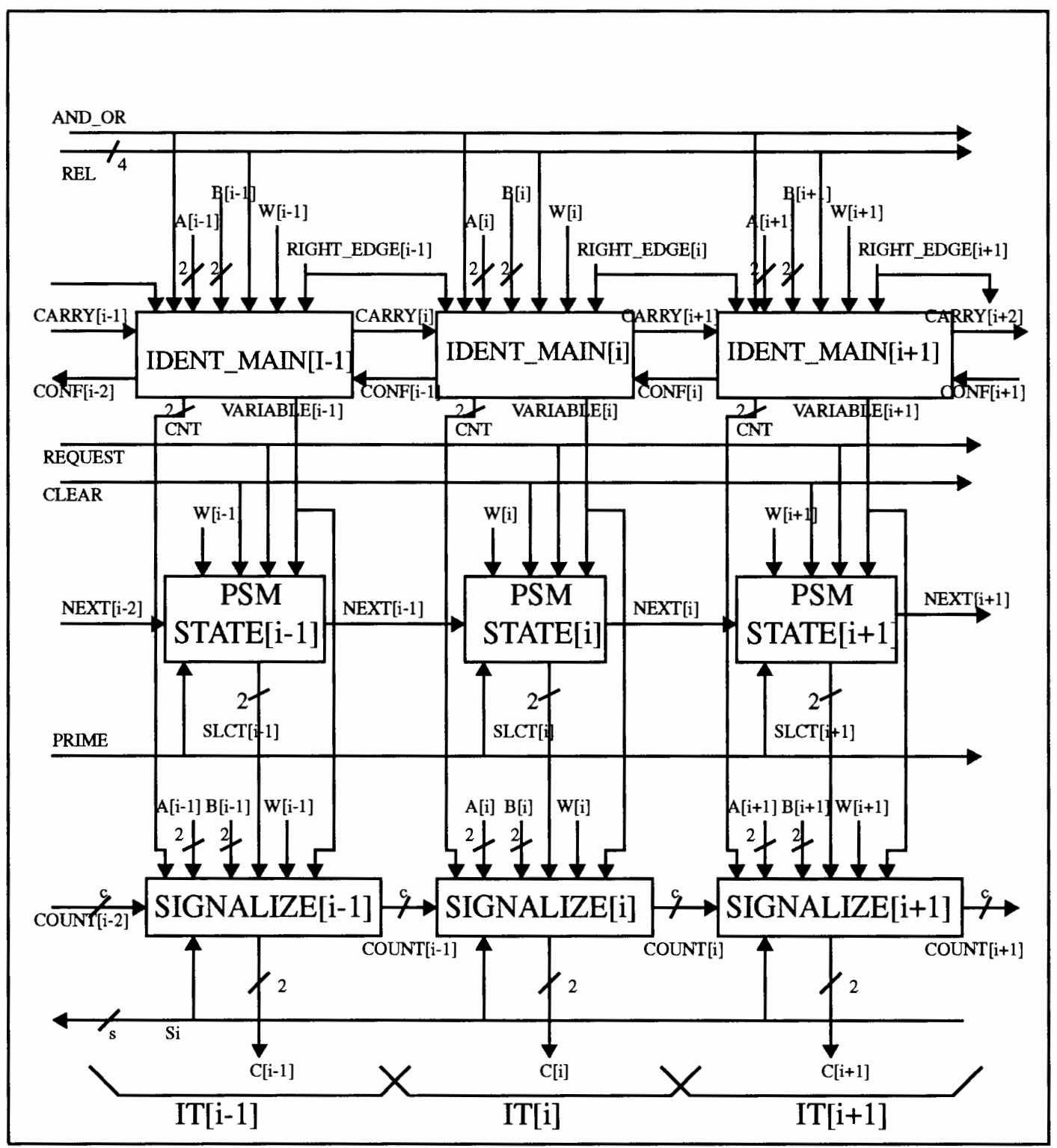

Figure 20: Details of IT and interconnections of ITs in the ILU 


\subsubsection{The Structure of a Single IT Cell}

Figure 21 shows the functional diagram of a single IT cell.

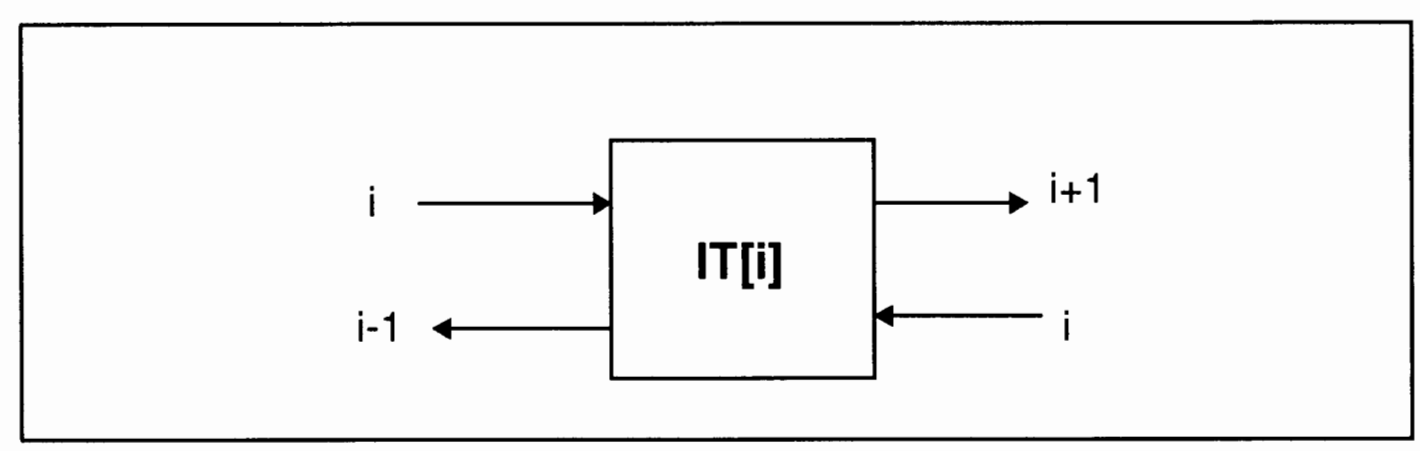

Figure 21: The numbers of iterative signals

The local signals in block $i$ have index $i$. The principles of enumeration of iterative signals are:

- To the block " $i$ " comes signal with index " $i "$.

- The iterative signal leaving block " $i$ " is either " $i-l$ " or " $i+l$ ".

- If the iterative signal goes to the left (to ITs of smaller numbers) then index " $i-1$ " corresponds to it.

- If the iterative signal goes to the right (to ITs of higher numbers) then index " $i+l$ " corresponds to it.

Figure 24 shows the function diagram of a single IT. In this Figure, the rel, bef, act, and aft signals are global for all ITs in the ILU. They come from the Bus Interface Unit. They are stable, set to 0's or 1's and describe the relation or operations to be executed on $A_{0}[i]$ 's, and $B_{0}[i]$ 's, as well as on $A_{1}[i]$ 's and $B_{1}[i]$ 's.

All horizontal signals are connected to neighbors of given IT (like CONF[i] or NEXT $[i+1]$ ) or to the fields of the registers corresponding to the given IT (like $A_{0}[i]$, $\left.B_{1}[i]\right)$

Vertical signals are global, i.e. they go either to/from the registers (like AND_OR) or 
are combined globally (like READY[i]).

\subsubsection{The IDENT_MAIN Block}

The bock IDENTIFY is composed of the block IDENT_MAIN, the block STATE and two multiplexers to create signals REL0 and REL1. The block STATE is a state machine which determines whether the literal to which the IT belongs is in the before, active, or after state. The block IDENT_MAIN uses the iterative signals CARRY and CONF to determine whether the IT is part of a specific literal.

CARRY and CONF are two iterative signals from left to right and from right to left, respectively.

If the relation is of AND type, then

$$
C A R R Y[i+1]=\left\{\begin{array}{l}
R E L 0[i] \cdot R E L 1[i] \quad \text { if IT[i] is the first IT of a variable } \\
C A R R Y[i] \cdot R E L 0[i] \cdot R E L 1[i] \quad \text { otherwise }
\end{array}\right.
$$

If the relation is of OR type, then

$$
C A R R Y[i+1]=\left\{\begin{array}{l}
R E L 0[i]+R E L 1[i] \quad \text { if IT[i] is the first IT of a variable } \\
C A R R Y[i]+R E L 0[i]+R E L 1[i] \quad \text { otherwise }
\end{array}\right.
$$

For both type of operation,

$$
\operatorname{CONF}[i-1]=\left\{\begin{array}{c}
\operatorname{CARRY}[i+1] \\
\operatorname{CONF}[i]
\end{array} \quad \text { otherwise } \mathrm{IT}[\mathrm{i}]\right. \text { is the last IT of a variable }
$$

If It[i] is the last IT of a variable, then RIGHT_EDGE[i]=1; otherwise,

\section{RIGHT_EDGE[i] $=0$.}

If IT[i] is the first IT of a variable, then RIGHT_EDGE[i-1]=1. Because IT[1] is always the first IT of a variable, we set RIGHT_EDGE[0]=1.

The equations describing the IDENT_MAIN block of the i-th IT cell are as follows:

$$
\begin{aligned}
& C A R R Y \_A N D[i+1]=R E L 0[i] \cdot R E L 1[i] \cdot R I G H T \_E D G E[i-1] \\
& +(C A R R Y[i]+R E L 0[i]+R E L 1[i]) \cdot \overline{R I G H T \_E D G E[i-1]} \\
& \text { CARRY_OR }[i+1]=(R E L 0[i]+R E L 1[i]) \cdot R I G H T \_E D G E[i-1]
\end{aligned}
$$




$$
+(C A R R Y[i]+R E L 0[i]+R E L 1[i]) \cdot \overline{R I G H T \_E D G E[i]}
$$

CARRY $[1]=1$

CARRY[I] is a don't care signal which can be set to either 0 or 1 .

RIGHT_EDGE[O] $=1$

$C A R R Y[i+1]=C A R R Y \_A N D[i+1] \cdot A N D \_O R+C A R R Y \_O R[i+1] \cdot \overline{A N D \_O R}$

CONF $[i-1]=$ CARRY $[i+1] \cdot R I G H T \_E D G E[i]$

$$
+ \text { CONF }[i] \cdot \overline{R I G H T \_E D G E[i]}
$$

$\operatorname{CONF}[n]=0$

CONF[n] is a don't care signal too which can be set to either 0 or 1 .

VARIABLE[i] $=$ CONF $[i-1]$

The signal AND_OR=1 indicates that the operation is of AND type, while AND_OR $=0$ indicates that the operation is of the OR type. Since CARRY_OR always equals 1 whenever CARRY_AND equals 1 . So we get

$C A R R Y[i+1]=C A R R Y \_A N D[i+1]+C A R R Y \_O R[i+1] \cdot \overline{A N D \_O R}$

The equations for CARRY_OR[i+1] and CARRY_AND[i+1] can also be simplified. After simplification, we have:

$$
\begin{aligned}
\text { CARRY_OR }[i+1]= & R E L 0[i]+R E L 1[i]+C A R R Y[i] \cdot \overline{R I G H T \_E D G E[i-1]} \\
\text { CARRY_AND }[i+1] & =R E L 0[i] \cdot R E L 1[i] \cdot R I G H T \_E D G E[i-1] \\
& + \text { CARRY }[i] \cdot R E L 0[i] \cdot R E L 1[i]
\end{aligned}
$$

If we now consider the signal of Water Register, W[i], we obtain the following set of equations which specify the signals inside the IDENT_MAIN block.

$$
\begin{aligned}
C A R R Y[i+1]=\overline{W[i]} & (C A R R Y[i] \cdot R E L 0[i] \cdot R E L 1[i] \\
& +R E L 0[i] \cdot R E L 1[i] \cdot R I G H T \_E D G E[i-1] \\
& +C A R R Y[i] \cdot \overline{R I G H T \_E D G E[i-1]} \cdot \overline{A N D \_O R} \\
& \left.+(R E L 0[i]+R E L 1[i]) \overline{A N D \_O R}\right) \\
+W[i] & \cdot C A R R Y[i]
\end{aligned}
$$




$$
\begin{aligned}
\text { CONF }[i-1] & =\overline{W[i]}\left(C O N F[i] \cdot \overline{R I G H T_{-} E D G E[i]}\right. \\
& \left.+\operatorname{CARRY}[i+1] \cdot R I G H T_{-} E D G E[i]\right)+W[i] \cdot C O N F[i]
\end{aligned}
$$

CARRY[1] $=1$

CONF $[n]=0$

CARRY[1] and CONF[n] are don't care signals, they could be set to either 0 or 1 . $R I G H T \_E D G E[0]=1$

$$
\begin{aligned}
\text { CNT0 }[i] & =R E L 0[i] \cdot R E L 1[i] \cdot A N D \_O R+(R E L 0[i]+R E L 1[i]) \cdot \overline{A N D \_O R} \\
& =R E L 0[i] \cdot R E L 1[i]+(R E L 0[i]+R E L 1[i]) \cdot \overline{A N D \_O R} \\
\text { CNT1 }[i] & =R I G H T \_E D G E[i] \cdot V A R I A B L E[i]
\end{aligned}
$$

The meaning of the signals used in the above equations is as follows:

\section{RELO[i], REL1[i]}

These are partial relations. For AND type operations, if both REL0[i] and REL1[i] are 1 , the relation of IT[i] is satisfied. For OR type operations, either RELO[i] or REL1[i] is 1 , the relation of IT[i] is satisfied.

\section{AND OR}

This signal equals 0 for OR-type relation. AND_OR $=1$ for AND-type relation.

\section{CARRY[i+1]}

This is a carry signal passed to the next IT for combining partial relations for literals of more than two possible values. It is adjusted according to the contents of the Water Register. Whenever W[i] is 1 , given IT is not supposed to perform any processing, but be transparent to all horizontal signals (since the number of bits required to represent all literals in the argument cubes is usually less than the number provided by all ITs, some of the ITs are not used). In a specific position, the CARRY signals propagates a " 1 " to the last IT of the variable, both for AND-type and OR-type relations. The first 
meaningful CARRY signal is generated in the first IT assigned to a variable. In this IT, the value of the incoming CARRY[i] should not be taken into account for the value of CARRY[i+1]. Therefore the signal RIGHT_EDGE[i-1] is present in the equation for CARRY[i+1].

\section{CONF[i-1]}

This is the confirmation signal accounting for Water Register contents; on right edge (i.e. in the last IT assigned to given variable) incoming CONF[i] must not be taken into account. When RIGHT_EDGE[i] = 1 then the value of CONF[i-1] equals to CARRY [i+1], otherwise it takes the previous CONF[i] from the right.

\section{VARIABLE[i]}

The signal CONF[i-1] equal 1 means that the relation is true for this variable, so this variable is specific, VARIABLE[i] should be 1 . This IT is within a variable for which the relation on literals described by function rel is satisfied.

\section{RIGHT EDGE[i]}

This is a "right edge of a variable" signal. It simply indicates that the IT of number $i+1$ is assigned to another literal.

\section{CNT0}

This signal is used to count the number of ITs within which the function rel is satisfied.

\section{CNT1}

This signal is used to count the number of specific positions of an operation. This is also the number of resultant cubes. 


\subsubsection{The STATE Block}

The state transition diagram of the STATE block is shown in Figure 22. All transitions are performed synchronously on the active edge of the clock, REQUEST, except when signal CLEAR comes. The signal CLEAR resets all states to the before state asynchronously.

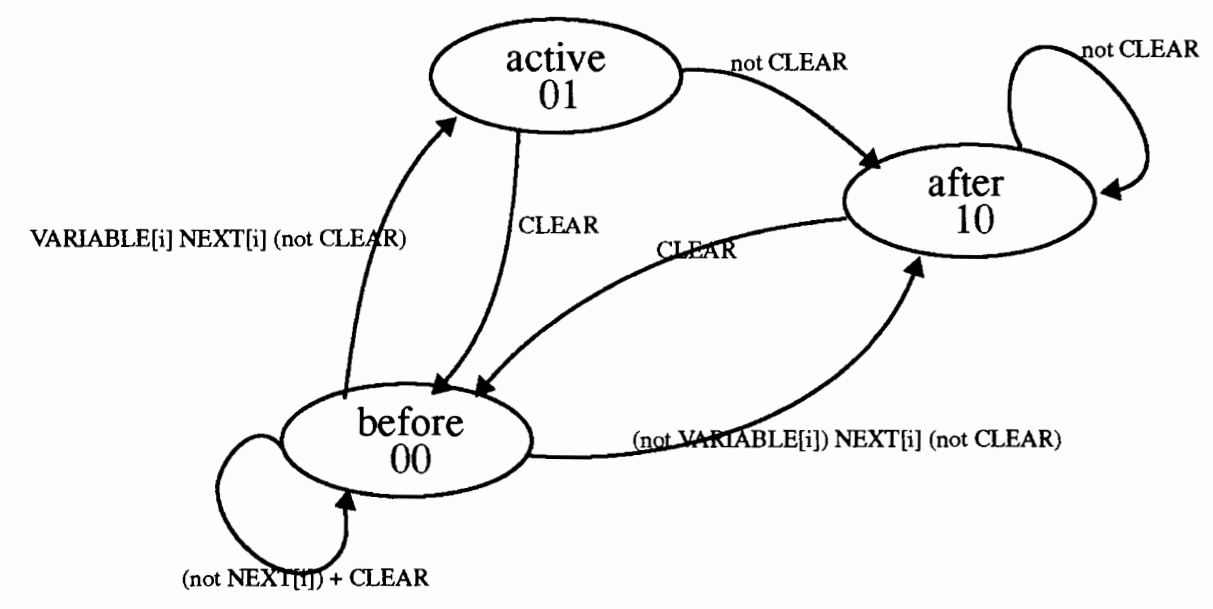

Figure 22: State transitions of the STATE block

Since there are three states, this state machine can be realized by using two D flipflops. The Q outputs of these two D flip-flops are named STATE0[i] and STATE1[i]. Inputs $\mathrm{D}$ are donated by EX0[i] and EX1[i], respectively. The realization of this state machine can be described by the following set of equations:

$$
\begin{aligned}
& \text { BEFORE }[i]=\overline{\text { STATE0 }[i]} \cdot \overline{\text { STATE } 1[i]} \\
& \text { ACTIVE }[i]=S T A T E 0[i] \cdot \overline{\text { STATE } 1[i]} \\
& \text { AFTER }[i]=\overline{\text { STATE0 }[i]} \cdot S T A T E 1[i] \\
& \text { EX0 }[i]=\text { BEFORE }[i] \cdot V A R I A B L E[i] \cdot N E X T[i]
\end{aligned}
$$


$\operatorname{EX} 1[i]=B E F O R E[i] \cdot \overline{\operatorname{VARIABLE}[i]} \cdot N E X T[i]+\operatorname{ACTIVE}[i]+\operatorname{AFTER}[i]$

$=\overline{\text { STATEO }[i]} \cdot \overline{\text { VARIABLE[i] }} \cdot N E X T[i]+A C T I V E[i]+A F T E R[i]$

The other signals that coming out of the STATE block can be described by the following equations:

$$
\begin{aligned}
\begin{aligned}
N E X T[i+1]=\overline{W[i]} & \left(N E X T[i] \cdot \overline{R I G H T \_E D G E[i] \cdot V A R I A B L E[i]}\right. \\
& +A C T I V E[i])+W[i] \cdot N E X T[i]
\end{aligned} \\
R E A D Y[i]=\overline{R E Q U E S T} \cdot R I G H T \_E D G E[i] \cdot N E X T[i] \cdot V A R I A B L E[i]
\end{aligned}
$$

SLCT[i] is a two-bit variable with SLCT0[i] as its least significant bit and SLCT1[i] as its most significant bit.

$$
\begin{aligned}
& \text { SLCT0 }[i]=\text { VARIABLE }[i] \cdot P R I M E+S T A T E 0[i] \cdot \overline{P R I M E} \\
& \text { SLCT1 }[i]=\text { STATE } 1[i] \cdot \overline{\text { PRIME }}
\end{aligned}
$$

Following are the explanation of signals in the above equations:

\section{NEXT[i+1]}

Essentially, position of active literal moves from one specific literal to the next one when the next resultant cube is going to be calculated. In order to avoid unnecessary delays, signal NEXT[i], propagated along IT chain during the processing of input cubes, prepares ITs supposed to transit to active state. Therefore, the transition to the active state can be done "immediately" (i.e. in the time determined by delays inside single IT) with coming of the active edge of the REQUEST clock. The equation of NEXT[i+1] means that NEXT[i] starts propagation to the right and it is propagated until RIGHT_ED[i] = 1 and VARIABLE[i] $=1$. So on the last IT[j] of next specific position NEXT[j+1] will be 0 ; in all previous IT[i]s, NEXT[i+1] will be 1 . 


\section{READY[i]}

The signal READY[i]=1 means that the rippling NEXT[i] signal has reached its destination and the next specific position is ready to be activated. The equation means: IT on the right edge of a specific variable has the active NEXT[i] signal on its input and this happens after the REQUEST clock has been activated. The signal REQUEST must be considered in the equation, because the remaining part of the equation for READY[i] is true all the time after NEXT[i] reaches one of the specific positions. When an IT[i] becomes active it sends NEXT[i+1] signal, rippling to the next specific group of ITs (i.e. ITs assigned to a specific variable). The NEXT[i] signal on the left side of currently active IT becomes low as soon as its low state ripples from the previously active IT (now that IT is in the after state).

\section{$\underline{\text { SLCT }[i]}$}

The SLCT[i] signal is used to select the appropriate function to be performed by a its IT: bef, act, or aft.

\section{$\underline{\text { PRIME }}$}

When the cube calculus to be performed is prime operation, $\mathrm{PRIME}=1$; otherwise, PRIME $=0$. Because only one resultant cube is generated in prime operation, function act should be performed on all the specific positions at the same time. Signal Prime presents in the equations for SLCT0[i] and SLCT1[i].

\subsubsection{The SIGNALIZE Block}

The structure of the SIGNALIZE block is shown in Figure 24.

A part of the resultant cube, C[i], is calculated in the SIGNALIZE block. A multiplexer is used to switch between the functions, bef, act, and aft. The bits SLCT0 and SLCT1 from the STATE block are the address inputs of this multiplexer, with SLCT1 as the most significant bit. When SLCT0=SLCT1 $=0$, the function $b e f$ is selected; when 
SLCT0 $=1$ and SLCT1 $=0$, the function act is selected; and when SLCT0 $=0$ and SLCT1 $=1$, the function aft is selected.

The selected function is placed at the data inputs of two 4 to 1 multiplexers with $\mathrm{A}[\mathrm{i}]$ and $B[i]$ as the address inputs. Remember, $A[i]$ and $B[i]$ each includes two bits of the operand cubes $\mathrm{A}$ and $\mathrm{B}$, respectively.

The circuit at the bottom of the SIGNALIZE block is a counter. The signal COUNT_SEL is used to select two counter modes. When COUNT_SEL=0, signal CNT0[i] goes into the COUNTER block. CNTO[i] is generated from the IDEN_MAIN block. It equals " 1 " in every IT for which the relation is satisfied. So in this mode, the number of ITs for which the relation is satisfied is counted. When COUNT_SEL=1, signal CNT1[i] goes to the COUNTER block. CNT1[i] equals "1" only in the last IT of a specific literal. So the number of specific literals, or in other words, the number of resultant cubes is counted.

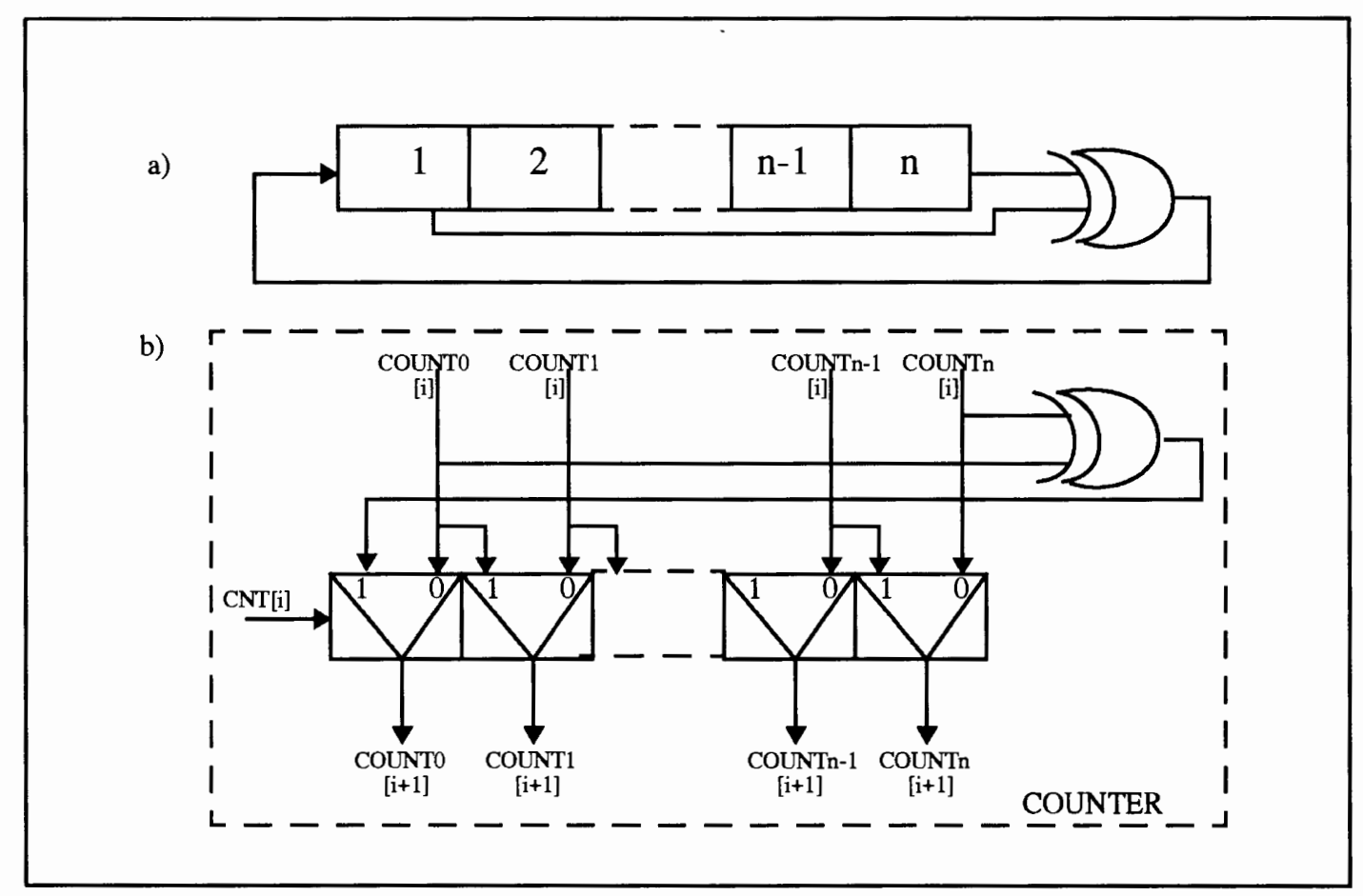

Figure 23: The iterative counter 
When $\mathrm{W}[\mathrm{i}]=1$ which means the $i$-th IT is not used, the signal that goes into the CNT input of the COUNTER block is always 0 , then the COUNTER block in IT[i] will pass the iterative count signal unchanged.

To minimize the necessary logic for the COUNTER block, the COUNTER block is implemented as a pseudo-random counter. It is based on a pseudo-random binary sequence generator that consists of a shift register and an XOR gate, as shown in Figure 23a. The count begins with the most significant bit equal to one. As the bits are shifted, a series of unique number will be generated. Figure 23b shows the implementation as an iterative counter, with multiplexers. The signal CNT0[i] or CNT1[i] determines whether the multiplexer will pass the iterative count signals unchanged to the next IT, or shift one bit to the left. A decoder outside the ILU is needed to decode the count signals from the last IT into a binary number before they are sent to the $\mathrm{CU}$. The number of iterative count signals depends on the number of ITs inside the ILU. 


\section{CHAPTER V}

\section{TESTABILITY DESIGN AND TESTABILITY ANALYSIS}

The testability design and analysis on the Iterative Logic Unit (ILU) of the Cube Calculus Machine (CCM) is accomplished in this thesis. Due to the time constraint, the Control Unit of the CCM is not designed. However, by using the testability design strategies presented in this thesis, a highly testable Control Unit can be designed, and then can be integrated with the ILU to obtain a highly testable CCM. This will be discussed in section 5.3.6.

Mentor Graphics EDA tools were used in the testability design and analysis of the ILU. The reasons that Mentor Graphics tools were chosen are:

- They are widely used in industry.

- They are installed in the EE computer system of Portland State University and are supported by Mentor Graphics.

\subsection{Design Capture}

Design Architect (v8.4_1.2) from Mentor Graphics was used in capturing the ILU. Design Architecture is a computer-aided schematic capture tool. It can be used to capture both schematics and symbols. Design Architect supports bottom-up design capture method.

The schematics and symbols captured using Design Architecture are in Electronic Design Data Model (EDDM) netlist format. EDDM netlist format is a Mentor Graphics internal netlist format which is an acceptable netlist format for most Mentor Graphics 
tools including FastScan, an ATPG tool which was used in the testability analysis of the ILU. A netlist in EDDM format can be converted to a netlist in EDIF format (Electronic Design Interchange Format), which is an industrial standard netlist format, using ENWrite from Mentor Graphics.

The primitive models used in the ILU were taken from Mentor Graphics Digital General Library.

The ILU was captured in a bottom-up fashion. The schematics of the lower level design blocks were captured first, then their symbols were generated, and later these symbols were used in the schematics of upper level design blocks. Figure 24 shows the hierarchical structure of the IIU. The rectangular boxes in Figure 24 represent design blocks.

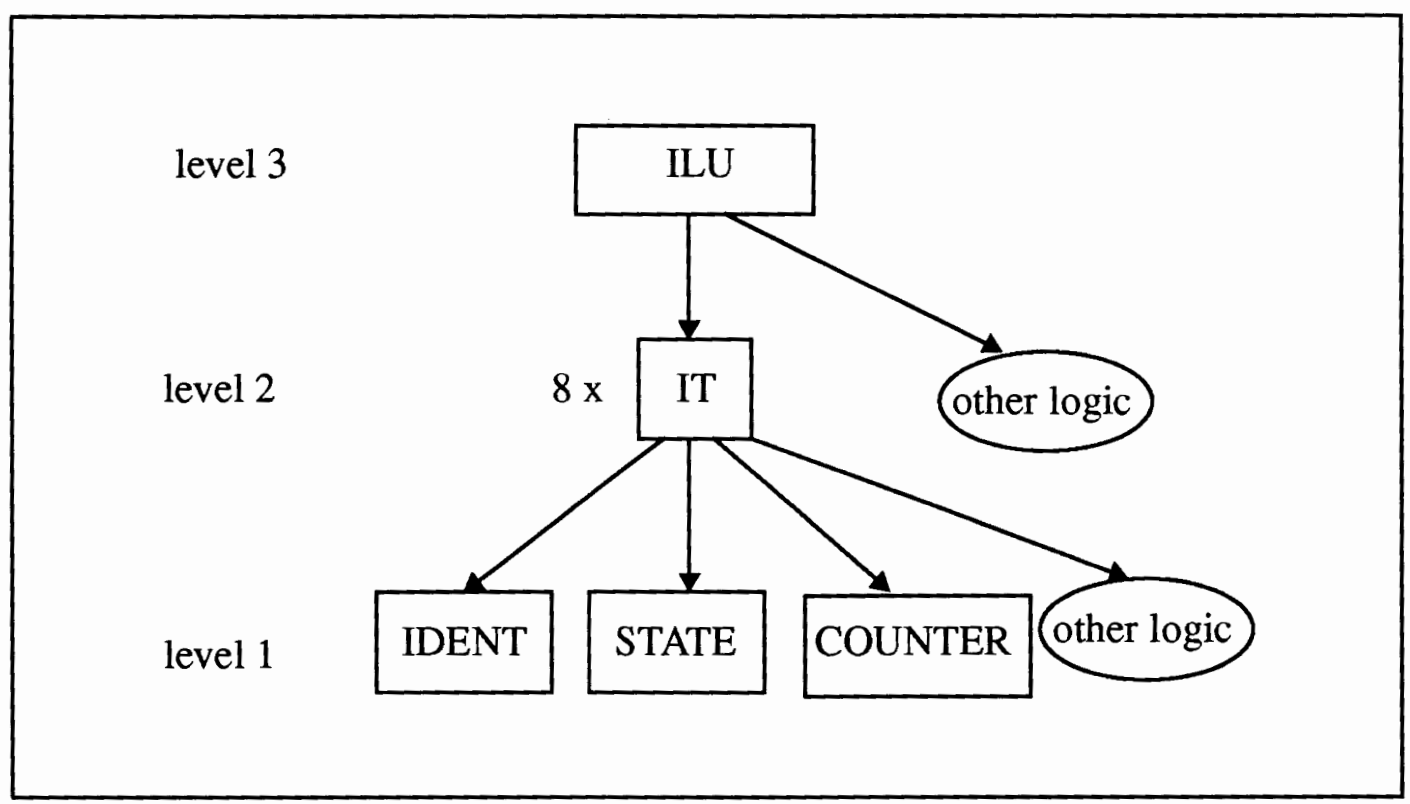

Figure 24: The hierarchical structure of the ILU

For simplicity, the ILU implemented here has eight IT instances. Since each IT can calculate 2 bits of the resultant cube, a total of 16 bits of the resultant cube can be calculated in this ILU, in other words, cube calculus operation on two eight-variable 
binary cubes can be performed in this ILU.

The ILU can be easily expanded by cascading more ITs to calculate wider cubes.

The IT was captured according to its functional description from Chapter 4.

The iterative count signals in this ILU is four-bit wide. As discussed in Chapter 4, the COUNTER block was implemented as a pseudo-random counter. A series of $2^{n}-1$ numbers of unique bit patterns can be generated on the iterative count signals, where $n$ is the width of the iterative count signals [27]. If all the literals of the operand cubes are binary, there can be, at most, eight specific positions in the ILU. The counter must be able to signal nine different numbers, to include the case where there is no specific position. Since the iterative count signal in this ILU is four-bit wide, the counter can signal 15 different numbers which is sufficient for an ILU with 8 IT cells. Notice, when expanding the number of ITs in the ILU to calculate wider cubes, the width of the iterative count signals should be expanded accordingly.

Details about how to use Design Architect to capture schematics and symbols can be found in Design Architecture User's Manual from Mentor Graphics.

The schematics and symbols of the ILU can be found in Appendix A.

A copy of the electronic design of the ILU is located in /u/mperkows/ccm on the EE computer system of Portland State University.

\subsection{Functionality Test}

After a design is captured, its functionality need to be fully tested to make sure that it does what it is supposed to.

Functionality test on the ILU was performed through simulation by using QuickSim II (v8.4_1.2) from Mentor Graphics.

Simulation is the behavior analysis of an electronic design without the costs of the physical hardware. QuickSim II reads in a design, calculates the behavior of a design 
and provides useful displays that can be used for analyzing the behavior of a design. It provides a reality check that gives a designer confidence in his design work.

The simulation of the ILU was performed in a bottom-up fashion. The simulations of the lower level design blocks were performed first, then the simulations of the higher level design blocks were performed. This way, the design bugs can be identified earlier and can be fixed easier. In our case, the STATE, the COUNTER, and the IDENT blocks were simulated first, then the simulation of the IT was performed, and the ILU was last simulated.

At each design block, a test plan was drafted to systematically test the functionality of that block.

The test plan on the ILU is given here.

The combinational operations were selected to be tested first. The combinational operations do not make use of the positional state machine within each IT. The operation always stays in the state of before for the entire operation, thus eliminating the problems that might caused by the positional state machine.

Once the combinational operations had been tested, the sequential operations were then tested. This class of operations uses the entire IT, including the positional state machine which was not used for the combinational operations.

Examples of the cube calculus operations that have been tested are listed below.

The force files of these examples are saved in $/ \mathrm{u} /$ mperkows/ccm/force_files on the EE computer system of Portland State University. A force file contains a set of QuickSim II commands which are used to force stimulus, list and trace desired signals.

\section{Supercube:}

Input cubes: $A=X^{12345} Y^{12}, B=X^{34} Y^{3456}$,

where $\mathrm{X}$ and $\mathrm{Y}$ are both eight-value variables.

ILU configurations: A: 0111110001100000 ;

B: 0001100000011110 ;

RIGHT_EDGE: 00010001; 
W: 00000000;

BEF: 0111;

REL: 1111.

Resultant Cube: C: 0111110001111110 , which is $X^{12345} Y^{123456}$

Force file: supercube.do

\section{Intersection:}

Input cubes: $A=X^{23456} Y^{123}, B=X^{45} Y^{2345}$,

where $\mathrm{X}$ and $\mathrm{Y}$ are both eight-value variables.

ILU configurations: A: 0011111001110000 ;

B: 0000110000111100 ;

RIGHT_EDGE: 00010001;

$\mathrm{W}: 00000000$;

BEF: 0001;

REL: 1111.

Resultant cube: C: 0000110000110000 , which is $X^{45} Y^{23}$.

Force file: intersection.do

\section{Crosslink}

Input cubes: $A=X^{0} Y^{1}, B=X^{2} Y^{3}$,

where $\mathrm{X}$ and $\mathrm{Y}$ are both four-value variables.

ILU configurations: A: 1000010000000000 ;

B: 0010000100000000 ;

RIGHT_EDGE: 01010000 ;

W: 00001111;

BEF: 0101;

ACT: 0111;

AFT: 0011; 
REL: 1110;

PRIME: 0;

AND_OR: 1;

TEST_MODE: 0.

Resultant cubes: $\mathrm{C} 1: 1010010000000000$, which is $X^{02} Y^{1}$;

C2: 0010010100000000 , which is $X^{2} Y^{13}$.

Force file: crosslink.do

\section{Symmetric Consensus}

Input cubes: $A=X^{01234} Y^{01}, B=X^{012} Y^{23}$,

where $\mathrm{X}$ and $\mathrm{Y}$ are both eight-value variables.

ILU configurations: A: 1111100011000000 ;

B: 1110000000110000 ;

RIGHT_EDGE: 000010001;

W: 00000000;

BEF: 0001;

ACT: 0111;

AFT: 0001;

REL: 1110;

PRIME: 0;

AND_OR: 1;

TEST_MODE: 0.

Resultant cubes: C: 1110000011110000 , which is $X^{012} Y^{0123}$.

Force file: sym_cons.do

\section{Sharp}

Input cubes: $A=U^{1234} V^{01} Z^{12}, B=U^{23} V^{01} Z^{23}$, 
where $\mathrm{U}$ is a eight-value variable, $\mathrm{V}$ is a binary variable, and $\mathrm{Z}$ is a six-value variable.

ILU configurations: A: 0111100011011000 ;

B: 0011000011001100 ;

RIGHT_EDGE: 00011001;

W: 00000000;

BEF: 0101;

ACT: 0100;

AFT: 0101;

REL: 0100;

AND_OR: 0;

PRIME: 0;

TEST_MODE: 0 .

Resultant cubes: C1: 0100100011011000 , which is $U^{14} V^{01} Z^{12}$;

C2: 0111100011010000 , which is $U^{1234} V^{01} Z^{1}$.

Force file: sharp.do

\section{Disjoint Sharp}

Input cubes: $A=U^{1234} V^{01} Z^{12}, B=U^{23} V^{01} Z^{23}$, where $\mathrm{U}$ is a eight-value variable, $\mathrm{V}$ is a binary variable, and $\mathrm{Z}$ is a six-value variable.

ILU configurations: A: 0111100011011000 ;

B: 0011000011001100 ; RIGHT_EDGE: 00011001;

W: 00000000 ;

BEF: 0101;

ACT: 0100;

AFT: 0001; 
REL: 0100;

AND_OR: 0;

PRIME: 0;

TEST_MODE: 0 .

Resultant cubes: C1: 0100100011011000 , which is $U^{14} V^{01} Z^{12}$;

C2: 0011000011010000 , which is $U^{23} V^{01} Z^{1}$.

Force file: disjoint_sharp.do

\subsection{Testability Design and Analysis}

There are two approaches to implement the CCM on hardware. One is to implement the CCM on programmable logic devices, like FPGA or CPLD. We call this approach the FPGA approach. The other is to implement the CCM as a Application Specific Integrated Circuit (ASIC). We call this approach the ASIC approach.

The FPGA approach is an excellent solution for prototyping the CCM. Since the programmable logic devices are reprogrammable, a design can be easily modify on these devices.

The ASIC approach has its own advantages. The size of a ASIC CCM chip can be much smaller than that of a FPGA CCM, which makes it easier to insert a CCM into a computer to perform as a co-processor. The cost of a ASIC CCM can be lower than the cost of a FPGA CCM if CCMs are produced in a relatively large quantity.

When the ASIC approach is chosen, we must make sure that the manufactured CCM chips can be fully tested to filter out manufacturing defects before the CCM design is handed over to an ASIC vendor. A testability analysis, and a testability design if needed should be performed on the CCM.

The testability design and analysis of the ILU part is presented in this section. 


\subsubsection{Fault Model and Design for Test Strategy}

Due to the complexity of the manufacturing process not all die on a wafer correctly operate. Small imperfections in starting material, processing steps, or in photomasking may result in bridged connections or missing features.

Manufacturing defects lead to particular circuit defects, including:

- nodes shorted to power or ground.

- nodes shorted to each other.

- inputs floating/outputs disconnected.

Fault models can be used to abstract the behavior of the manufacturing defects at the logical gate level.

The single stuck-at fault model was used in the testability analysis of the ILU.

As we recall from Chapter 2, the stuck-at fault model models a behavior that occurs if the terminals of a gate are stuck at either high (stuck-at-1) or low (stuck-at-0) voltage levels. Most physical defects that occur exhibit behavior that makes a node appear to be stuck at power or ground.

Single stuck-at fault model models the situation that there is one and only one stuckat fault occurred in a design. Using this fault model, while generating a test vector to detect a specific stuck-at fault, ATPG tool views the whole design as fault free except the current fault site. This simplifies the task of generating a test vector to detect this fault. Years of research has proven that the single stuck-at fault model is effective in finding many common defect types. It is same to say if a chip passes a set of test vectors which can detect every single stuck-at fault in the design, this chip will very unlikely to fail in the field. Single stuck-at fault model has become an industry standard. It is the most common fault model used in fault simulation. This is why single stuck-at fault model was used in our testability analysis.

Both stuck-at 0 and stuck-at 1 faults on the input and output pins of the netlist primitives in the ILU were analysed.

Full scan design strategy was employed in the testability design of the ILU which 
basically converts all the memory elements in the ILU to be scannable memory elements and stitches them together to be a scan chain.

Since the memory elements in the ILU are D flip-flops, mux-dff scan cells were selected to replace the $\mathrm{D}$ flip-flops in the original design. A mux-dff scan cell contains a single D flip-flop with a multiplexed input line that allows selection of either normal system data or scan data. Figure 29 shows the replacement of an original flip-flop with mux-dff scan cell.

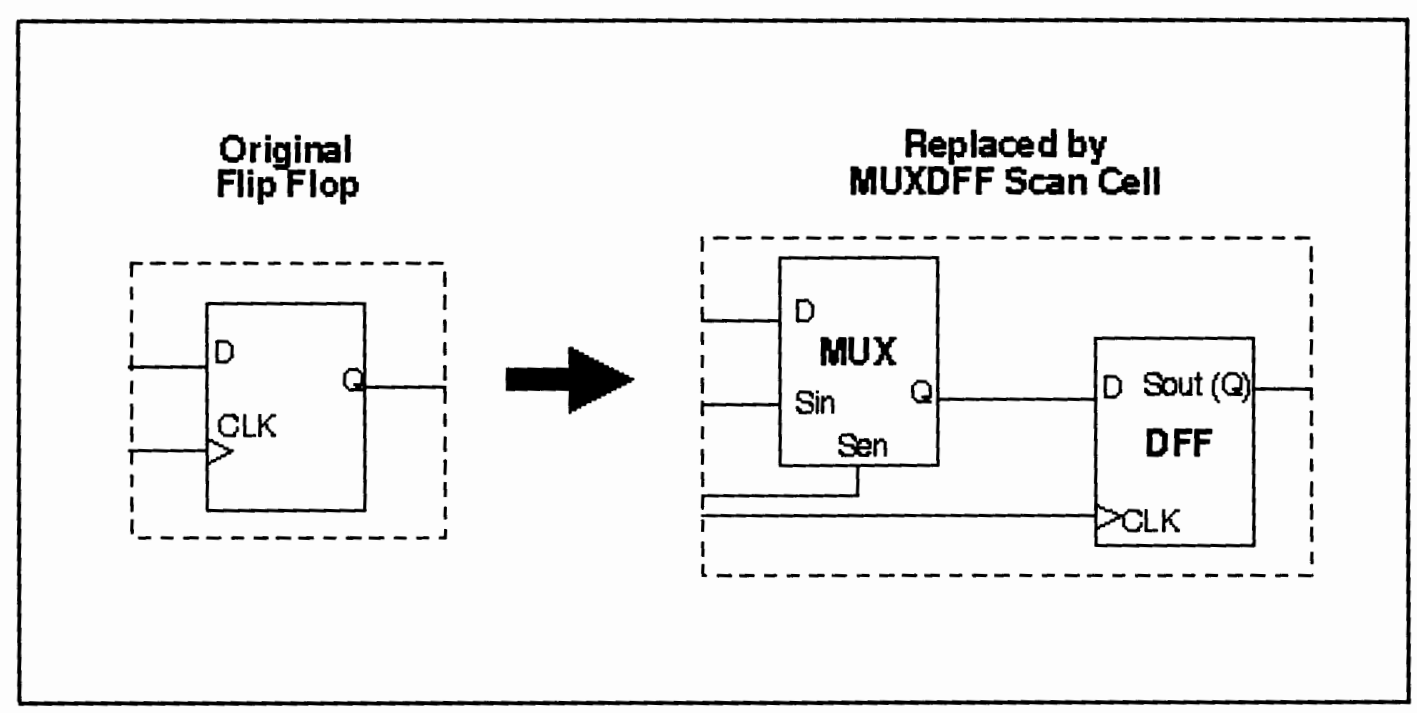

Figure 25: Mux-dff scan replacement

As we can see from Figure 25, the cost of converting a D flip-flop to a mux-dff scan cell is adding a 2-to-1 multiplexer. Since there are only two D flip-flops in each IT cell, full scan design does not have big impact on the timing and silicon space of the CCM chip. Instead, as we discussed in Chapter 3, full scan design ensures the CCM to become highly testable, as we will find later.

\subsubsection{FastScan}

FastScan (v8.4_2.18) which is a full scan ATPG tool from Mentor Graphics was cho- 
sen to help the testability analysis and the test pattern generation.

It has been proven that FastScan can generate high coverage test patterns for combinational or sequential circuitry. This is the reason that FastScan was chosen for CCM.

A brief introduction to FastScan is given here. The introduction here is not mean to be comprehensive. It only covers the features that were used in the testability design of the CCM. More detailed information on FastScan can be found in FastScan Reference Manual and ASIC/IC Design for Test Process Guide from Mentor Graphics.

\section{Features}

- Produces high coverage test patterns for full scan designs.

- Contains an internal high-speed fault simulator.

- Contains a design rules checker.

- Can compress test patterns.

\section{Inputs to FastScan}

- Design can be in EDDM, EDIF, GENIE, and TDL netlist format.

- Test procedure file defines the operation of the scan circuitry in the design.

- Library contains descriptions of all the cells used in the design. The library is used to translate the design data into a flat, gate level simulation model that is used by the fault simulator and test generator.

\section{Outputs from FastScan}

- Test patterns can be in a number of different simulator and ASIC vendor formats.

- Report file gives the test coverage, fault coverage, and the ATPG effectiveness for the test patterns generated.

\section{FastScan's ATPG method}

FastScan creates a set of test patterns by using either random test pattern or deterministic test pattern generation. 
FastScan initially produces a large number of random patterns and identifies patterns that are necessary to detect faults. It stores only those patterns in the test pattern set. A number of faults on the fault list can be detected by the random patterns, thus fewer faults are left on the fault list to be detected by deterministic test pattern generation. ATPG performance can be improved this way. The random pattern generation stops when a simulation pass fails to detect at least $1 \%$ of the remaining faults.

FastScan then performs deterministic test pattern generation which creates the test pattern that can guarantee detection of a given fault. The basic procedure for deterministic test pattern generation is to pick a fault from the fault list, create a pattern to detect the fault, perform the fault simulation of the pattern to make sure that the fault was detected by the pattern. The D-algorithm which is presented in Chapter 2 is used in FastScan.

If all possible choices are exhausted without a successful test pattern, then the fault is called ATPG-untestable fault.

FastScan uses an event-based timing model. The non-scan events used by FastScan are force $\_i$ (force a primary input), measure po (measure a primary output), capture_clock_on (turn on the capture clock), and capture_clock_off (turn off the capture clock). These events are grouped into test cycles which can not be modified by the user.

\section{System Modes}

FastScan has four system modes. They are: SETUP mode where circuitry behavior can be set up, ATPG mode where test pattern generation can be performed, FAULT mode where fault simulation can be performed, and GOOD mode where good simulation (simulation on design without fault effects) can be performed.

When FastScan is invoked, it automatically goes to SETUP mode to let the user specify the circuitry behavior. 


\section{Basic Operating Procedures}

1. Invoking FastScan:

\$MGC_HOME/bin/fastscan < design_name> [-lib < file_name $>$ ] [-nogui]

where option "nogui" turns the Sim View graphic user interface off.

2. Setting up the design behavior: input signal constraints can be defined here.

3. Defining scan groups with their test procedure files: a scan group is a set of scan chains that can be loaded and unloaded by the test procedures defined in a single test procedure file.

4. Defining scan chains: for each scan chain, the name of the scan chain, the name of the scan group in which the scan chain is placed, the scan input pin, and the scan output pin can be defined here.

5. Defining scan clocks: scan clocks are defined as any signals that can capture data into sequential elements (such as system clocks, sets, resets, and enables). The off-state of the scan clocks must be defined with the scan clocks. The off-state is the state in which clock inputs of latches are inactive and the edge-triggered devices are at the value before the capturing transition.

6. Changing the system mode to ATPG: FastScan flatterns the netlist, performs circuit learning analysis, identifies the scan chains, tests the test procedure files, and checks the scan clock rules at this stage. A summary message of these learnings and checkings is given at this stage.

7. Adding faults to the current fault list: the user can specify whether the faults on some specific pins should be added to the current fault list or faults on all pins should be added to the current fault list.

8. Running ATPG.

9. Compressing test patterns: FastScan has the capability of compressing the test patterns in order to reduce the number of test patterns without losing test coverage.

10. Saving test patterns: test patterns can be saved in a number of different simulator and ASIC vendor formats.

11. Exiting. 


\subsubsection{FastScan Fault classification}

FastScan uses the same fault classification as those we discussed in section 2.10. Since the understanding of the fault classification is essential to improve the testability of a design, discussion on several fault types that we encountered in the testability analysis on the ILU is given in this section.

\section{Detected by Simulation Faults}

Detected by simulation faults are faults that were identified as detected during ATPG by using fault simulation.

\section{Detected by Implication Faults}

Detected by implication faults were identified as detected during ATPG not by using fault simulation, but by analysis. These faults are located on the scan chain(s) which can be detected by loading and unloading the scan chain(s).

\section{Possible Detected Faults}

A fault is detected when the good machine has a 0 value and the faulty machine has a 1 value, or the good machine has a 1 value and the faulty machine has a 0 value. In some situations, the pattern to detect a fault may result in an X (either a 1 or 0 ) in the faulty machine. In this case, there is no "hard" detect. The effect of the fault cannot be predicted accurately by the simulator. The result may vary from chip to chip, depending on physical circuit parameters and the manufacturing process. This situation results in a potential, or possible, detection. FastScan gives a $50 \%$ credit on these faults which means a possible detected fault has a $50 \%$ chance to be detected in real chip testing. Possible detected credit is used in the calculation of the test coverage.

An example of possible detected fault is shown in Figure 26. In this example, there is a stuck-at 0 fault on the enable line. The pattern would force the line to a 1 to detect the fault. The good machine results in a 0 at the output, if the B input is forced to a 0 . 
However, the faulty machine results in an X. If the tester measures a 1 , the fault is detected. However, if the tester measures a 0 , the fault goes undetected. Thus, this fault is possibly detected.

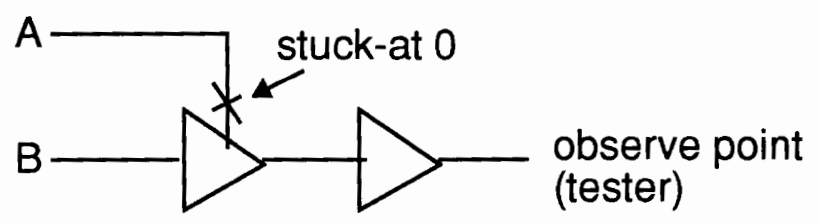

Figure 26: Example of possible detected fault

\section{ATPG Untestable Faults}

ATPG untestable faults includes those faults for which no pattern can be created to detected them, and yet still can not be proven redundant. There are various situations that FastScan identifies a fault as ATPG untestable. One example is when the sensitization path of a fault is blocked by an unknown state (FastScan treats the output of a nonscan memory element as unknown), this fault is classified as ATPG untestable. Another example is when an input pin is undriven, FastScan is not able to set a desired value on that pin. The faults on that input pin (both stuck-at 1 and stuck-at 0 ) are identified as ATPG untestable faults. We will see examples of these two types of ATPG untestable faults in the following testability analysis.

A design with poor testability usually has a large number of ATPG untestable faults. How to convert ATPG untestable faults to be detectable is a major task in testability design.

\section{Untestable Faults}

Untestable faults include unused, tied, blocked, and redundant faults.

Unused faults occur on pins that are not used. In the example shown in Figure 27 the 
QB output of the flip-flop is not connected. The faults that occur on this pin are classified as unused faults.

Tied fault include faults on nodes which are tied to a value identical to the fault values. In the example shown in Figure 27, stuck-at 0 faults on nodes A, B, C and D are tied.

Blocked faults include faults on circuitry for which all paths to an observable point are blocked by a tied logic. An example of blocked fault is shown in Figure 28.

Redundant faults are faults that are undetectable due to the redundant logic in the circuitry. No matter what pattern is applied, the faulty machine is always equivalent to the good machine because of the redundant logic. An example of redundant fault is shown in Figure 28.

From the above description of the untestable faults, we come to a conclusion that untestable fault will NOT cause any functional failure of a design. It is not included in the calculation of the test coverage. Untestable faults were ignored in the testability analysis of the ILU.

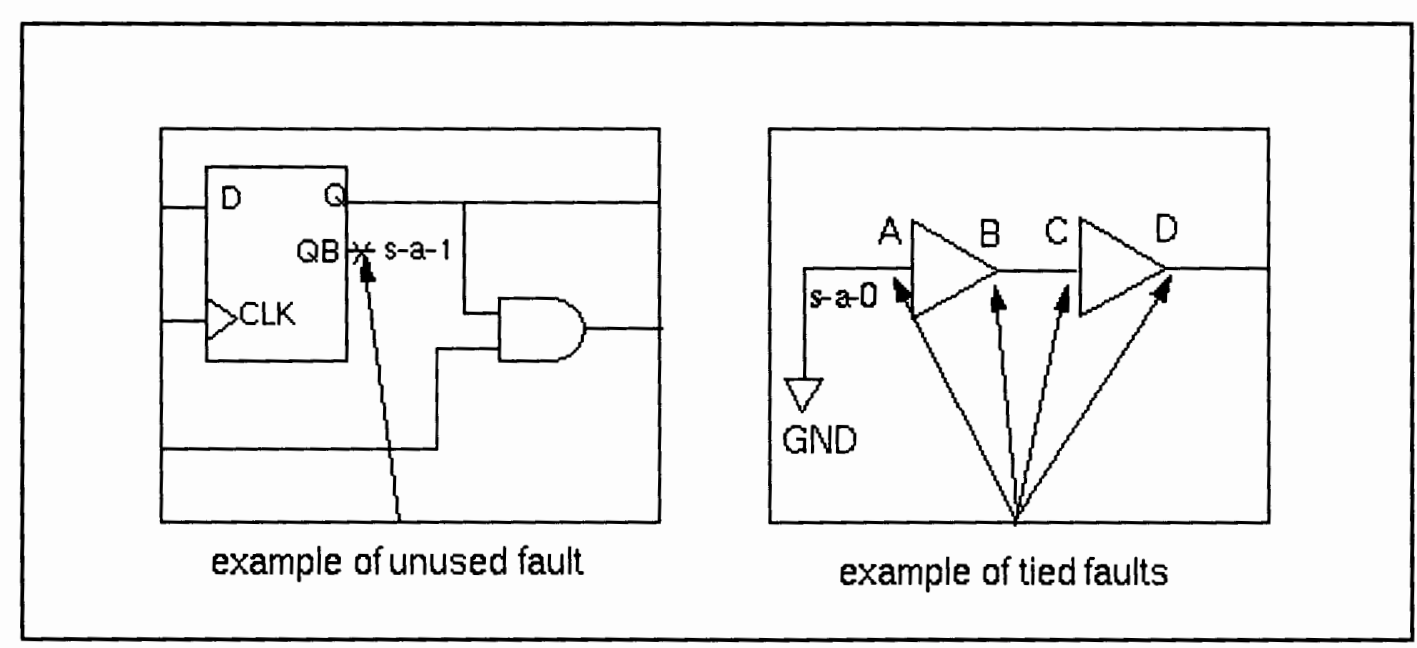

Figure 27: Examples of unused and tied faults 


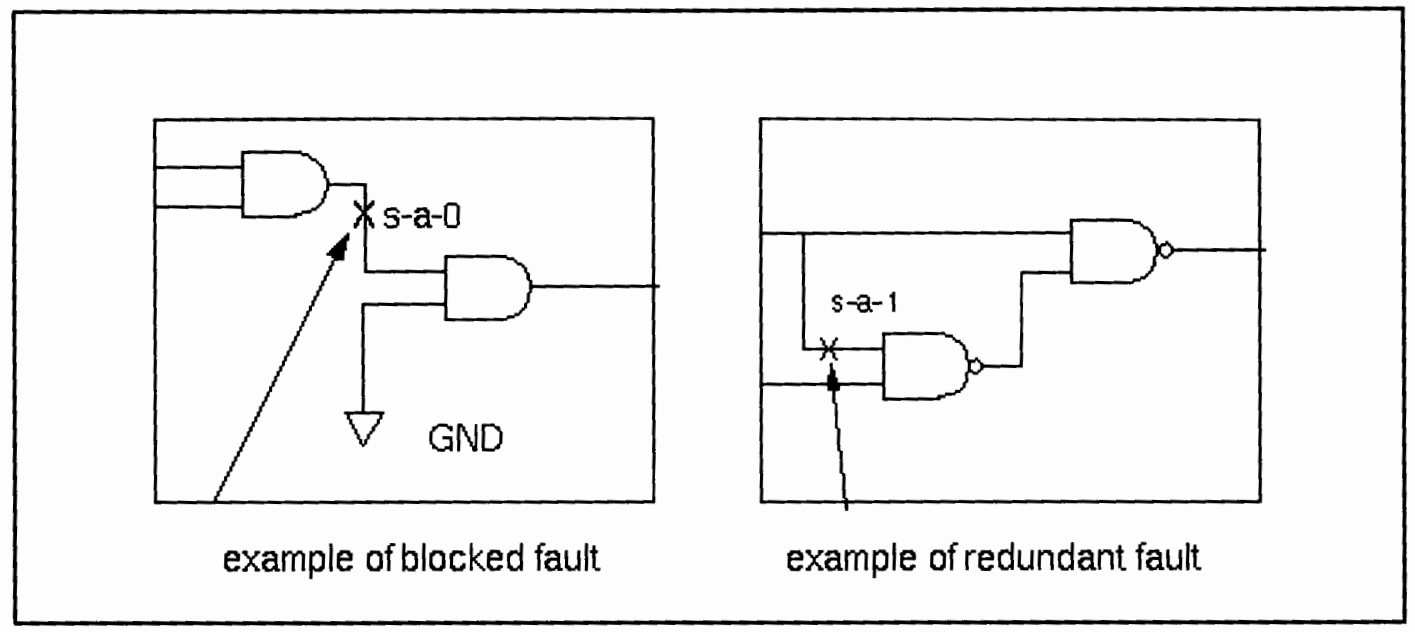

Figure 28: Examples of blocked and redundant faults

\subsubsection{Testability design and analysis}

Scan circuitry can be inserted automatically using test synthesis tools. DFTAdvisor from Mentor Graphics is one of these tools. DFTAdvisor supports both full scan and partial scan insertion. It supports the common scan methodologies, including muxscan, clocked-scan, and LSSD. It automatically generates scan setup and test procedure files for FastScan.

For illustration purposes, the scan circuitry in the ILU was inserted manually instead of using DFTAdvisor.

\section{A Novel Testability design approach}

As a part of his responsibilities for Mentor Graphics, the author has used several Mentor Graphics test synthesis and ATPG tools (BSDArchitect which is a test synthesis tool for boundary scan circuitry insertion, DFTAdvisor, and FastScan) for testability analyses of various industrial designs. One purpose of these testability design practices is to find ways to improve the testability of these design with least cost (minimizing the area and the timing impacts on the original design while inserting testing circuitry). Full scan, partial scan, and other testability design methods were used in these designs. 
The author has seen various testability problems and managed ways to solve these problems. Through these practices, the author also gained a good understanding of Mentor Graphics design-for-test tools. Based on these experiences, a novel testability design approach, a bottom-up approach, is proposed here.

Traditionally, the testability analysis and the testability design of a design is performed on a whole design. There are two disadvantages of this approach: first, it is very painful to identify the sources of testability problems on a whole design (a industrial design usually has more than thousands of gates); second, different design blocks within a design could have different circuit characteristics, applying one testability design method on the whole design usually doesn't achieve the best performance.

In the bottom-up approach, testability analysis and design are first performed on the lower level design blocks, and then move upwards. In each design block, testability of the original design of that block is evaluated by running ATPG. If a satisfied test coverage is obtained, then there is no testability problem with that design block and the block is left the way it was; Otherwise, testability problems within that design block is identified, appropriate testability design strategy is employed to remove the testability problems, and ATPG is performed on the modified design block to evaluate the testability of the modified design block. This process is repeated until a satisfied test coverage is achieved. This way, testability problems can be identified within each design block and be removed by using the most appropriate design-for-test method.

The bottom-up testability design approach is proposed based on the following two observations.

First, testability problems can be isolated and solved in each design blocks. Testability problems are actually controllability and observability problems. A fault is identified as untestable when this fault is uncontrollable, or unobservable, or both. If a design block has a test coverage of $100 \%$ and this design block (called lower level block) is instantiated in a higher lever design block, whether the faults in the lower level design block are testable or not in the higher level design block is only depends on the controllability of the input signals of the lower level design block and the observability of 
the output signals of the lower level design block in the higher level design block. If the input signals of the lower level design block are controllable and the output signals of the lower level design block are observable in the higher level design block, then all the faults within the lower level design block are still 100\% testable in the higher level design block. Any observability and controllability problems on the input and output signals of the lower level design block are testability problems of the higher level design block. This is illustrated in Figure 29. Thus, if a high testability is achieved on every design blocks, then a high testability can be expected on the whole design. An analogy that help understand this concept is to image each design blocks as procedures in software development. If every procedure (including the main program which compares to the top level design block in hardware design) are fully tested, then the whole program is guaranteed to work properly.

Second, by stitching scan chains inserted in different design blocks together or by adding direct accesses from the chip level, the testing circuitry inserted in every design blocks can be controlled from the chip level. Thus proper operations of the testing circuitry in each design blocks can be performed through control from the top level (chip level) during ATPG process.

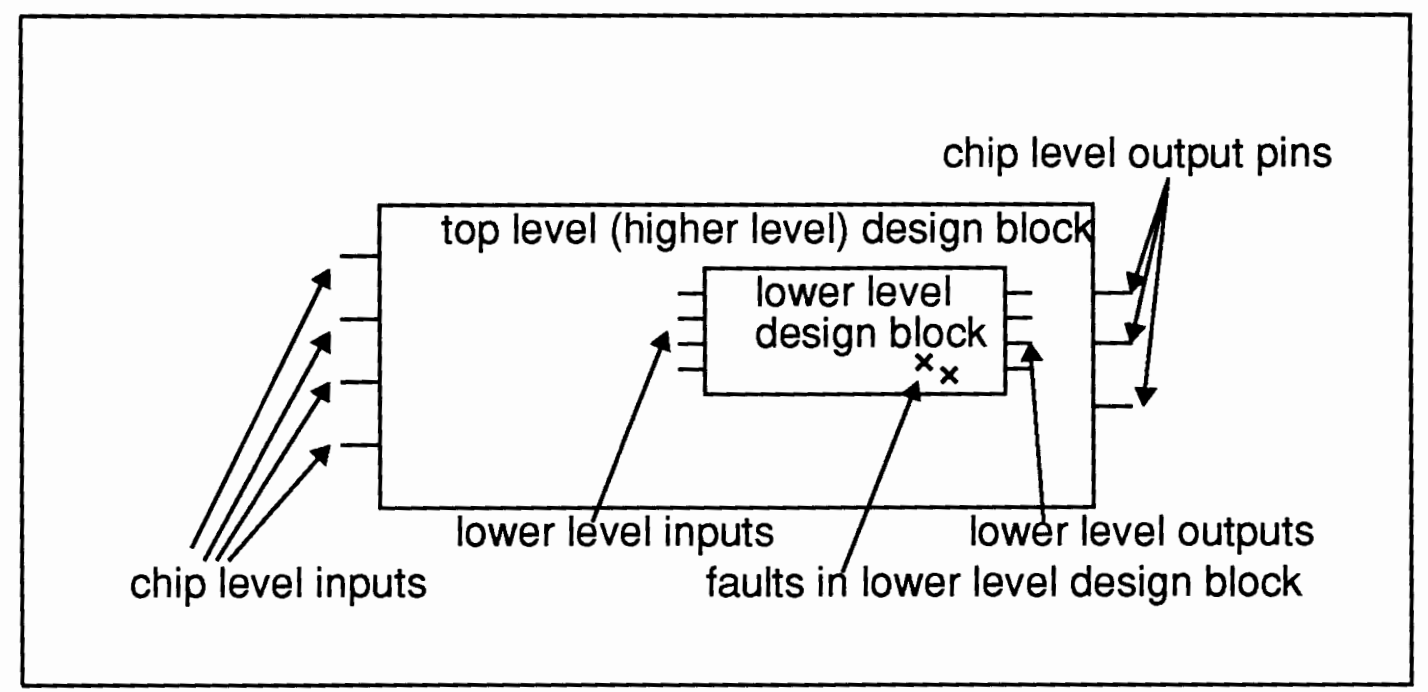

Figure 29: Diagram of lower and higher level blocks 
Testability design and analysis of the ILU was performed in this bottom-up fashion. Testability analyses and designs were first performed on the COUNTER, STATE, and the IDENT blocks which are the lowest level design blocks of the ILU, then the testability analysis on the IT cell was performed, and the testability analysis and design on the ILU was performed last.

\section{Testability Analysis of the COUNTER Block}

The COUNTER block is a combinational circuit. There is no special circuit behavior and scan information need to be set for running ATPG using FastScan.

The test patterns generated by FastScan for this block have a test coverage and fault coverage of $100 \%$ which means both stuck-at 0 and stuck-at 1 faults on the pins of the primitives used in this block are testable by using the test patterns generated by FastScan. There is no testability problem with this block at all. The COUNTER block is left the way it was.

A FastScan report file on the COUNTER block is shown is Figure 30. In this figure, "FU" stands for the total number of faults that were analysed; "DS" means the detected by simulation faults, the number listed on the right side of "DS" is the number of detected by simulation faults identified during the ATPG process; "\#test_patterns" is the number of test patterns in the final test pattern set; and "\#simulated_patterns" is the total number of test patterns simulated by FastScan fault simulation during the ATPG process; Since only the patterns that can detect a fault(s) are put in the final test pattern set, the "\#simulated_patterns" is always larger than the "\#test_patterns". A FastScan report file gives the test coverage, fault coverage, ATPG effectiveness and also the numbers of different types of faults identified in a design. The formulas for test coverage and fault coverage are given in section 2.11 . We notice that there is no detected by implication fault in this block, all the faults are detected by fault simulation. This is because there in no scan circuitry in this block. This is also true for the IDENT block. The transcript of FastScan running on the COUNTER block can be found in Appendix B 


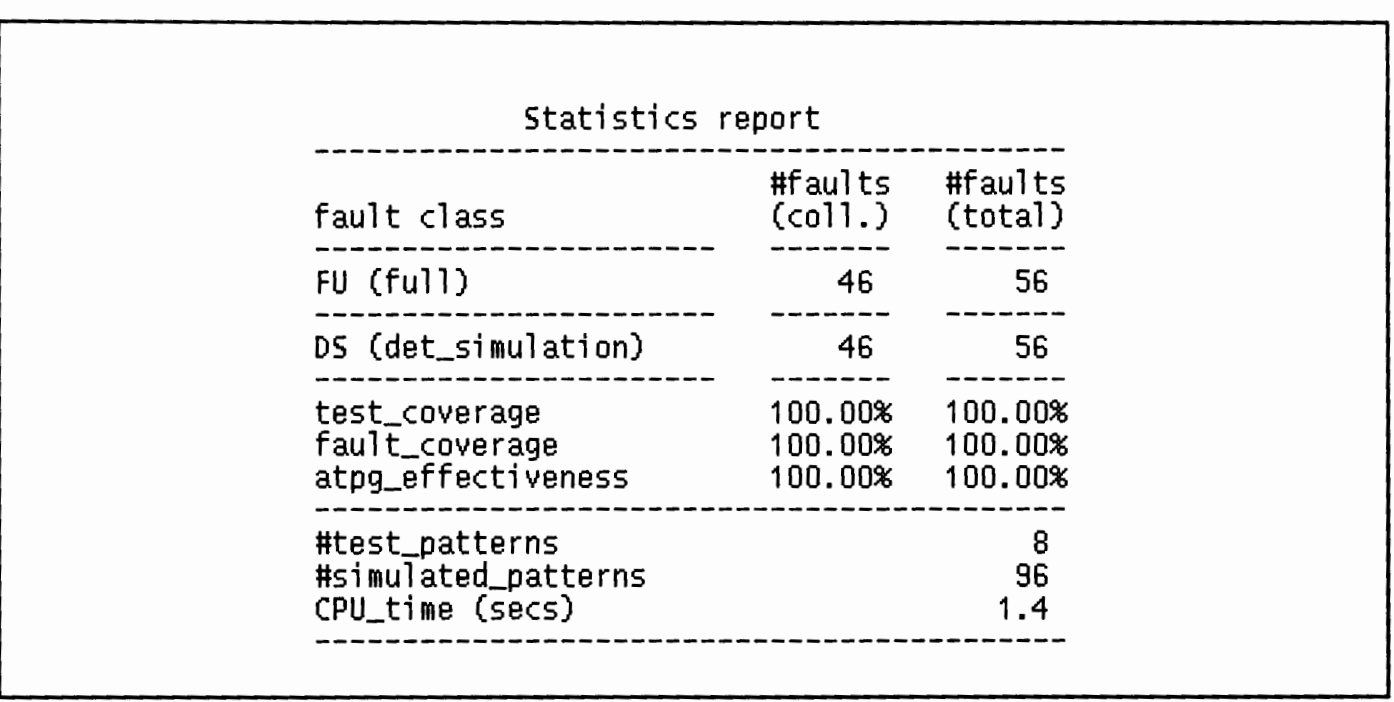

Figure 30: FastScan report on the COUNTER block

\section{Testability Analysis of the IDENT Block}

The IDENT block is also a combinational circuitry. There is no special circuitry behavior and scan information need to be set for running ATPG using FastScan.

The test patterns generated by FastScan for this block has a test coverage of $100 \%$ and a fault coverage of $100 \%$. No testability problem was found in the IDENT block and the IDENT block was left the way it was.

A FastScan report file on the IDENT block is shown in Figure 31. The transcript of FastScan running on the IDENT block can be found in Appendix B.

\section{Testability Analysis and Testability design of the STATE Block}

The STATE block is a sequential circuitry with two D flip-flops. A FastScan report file from the initial ATPG run on this block is shown in Figure 32.

The test coverage of the STATE block is only $24.44 \%$ before fault collapsing (A circuit contains a significant number of faults that behave identically to other faults. That is, the test may identify a fault, but may not be able to distinguish it from another fault. These faults are said to be equivalent, and the faults are therefore reduced to one equivalent fault in a process known as fault collapsing.). The large number of ATPG untest- 


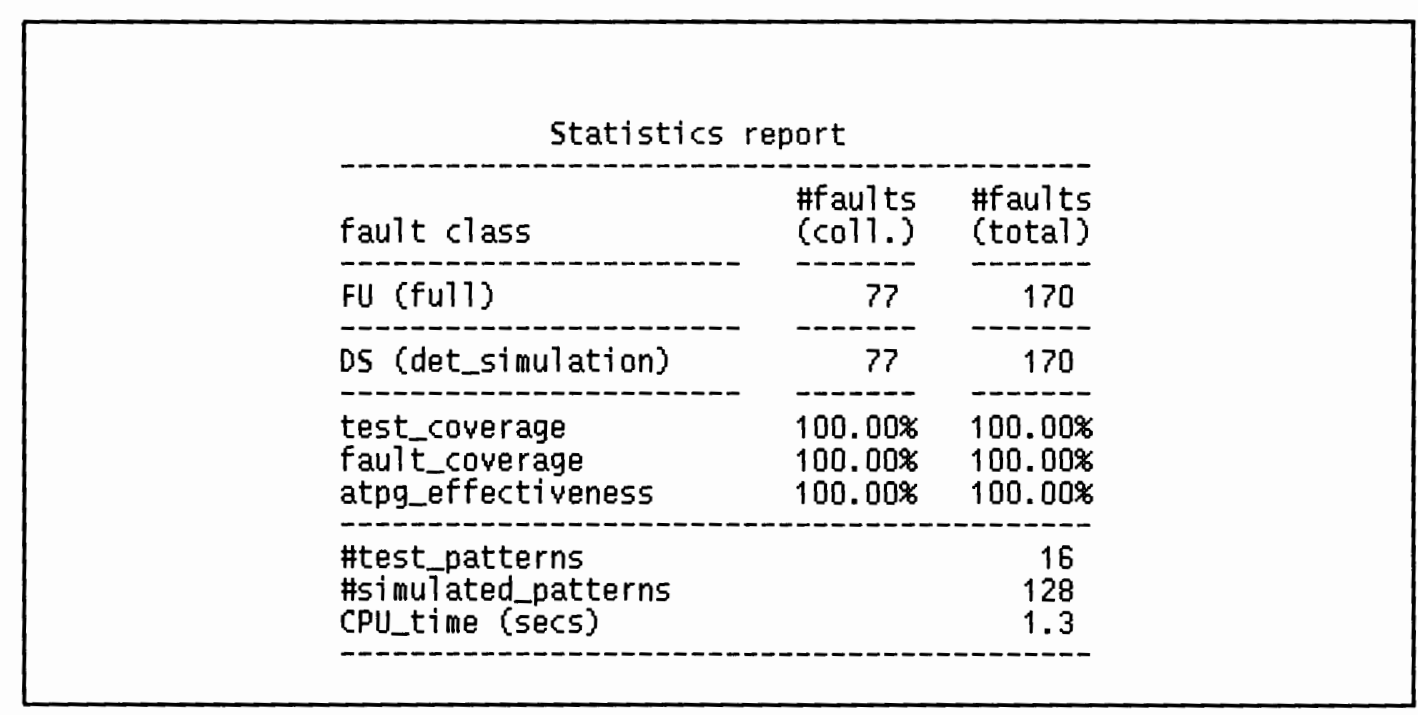

Figure 31: FastScan report on the IDENT block

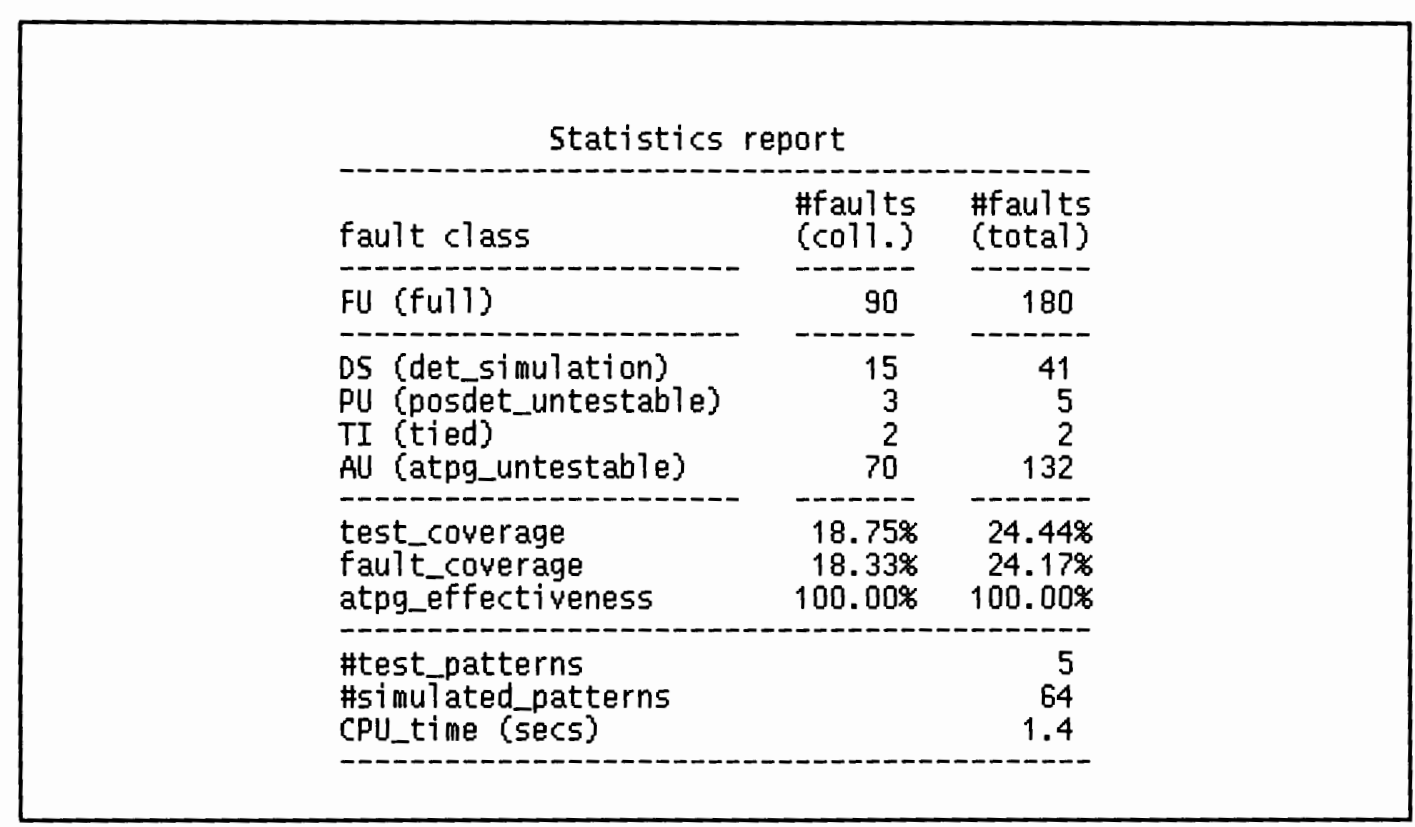

Figure 32: FastScan report on the STATE block

able faults in this block is the source of the low test coverage.

Due to the nature of the combinational ATPG algorithm used in FastScan, FastScan is not able to set a desired value on the outputs of a memory element without using the scan chain (FastScan has limited ability to deal with sequential circuitry without using scan if the memory elements in the sequential circuitry meet certain requirements. This feature is not discussed in this thesis). FastScan set the outputs of the non-scan mem- 


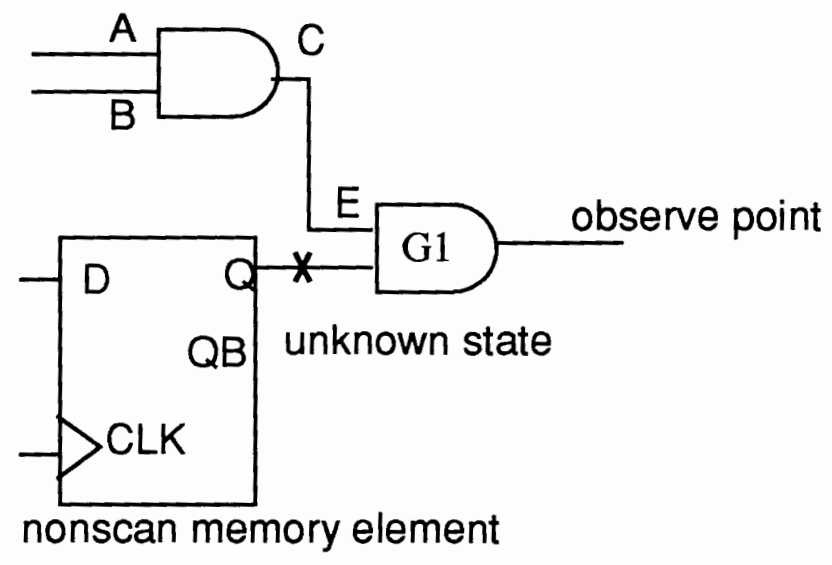

Figure 33: Example of ATPG untestable faults

ory element to unknown states. There are two D flip-flops in the STATE block. The outputs of these two D flip-flops were defined as unknown states by FastScan. These unknown states blocked the sensitization paths of a number of other faults. As shown in Figure 33, the fault effects of stuck-at 0 and stuck-at 1 faults on nodes A, B, C, and E can not be observed at the observe point because of the sensitization paths of these faults were blocked at gate $\mathrm{G} 1$ by the unknown state of the Q output of the non scan memory element. Since FastScan was not able to make the fault effects of these faults be observable at the outputs of this block, these faults were identified as ATPG untestable faults. Figure 34 shows all the ATPG untestable faults within the STATE block (little black box near a pin means there is an ATPG untestable fault on that pin.).

Scan design which is discussed in Chapter 3 can help us solve this problem. A full scan circuitry was inserted in the STATE block.

Figure 35 shows the D flip-flops in the STATE block before and after scan insertion. In this graph, signals $d l$ and $d 2$ are the normal system input data of D flip-flop $d f f l$ and $d f f 2$, respectively, signal scan_en is the scan mode enable signal, signal scan_in is the input of scan chain, and signal scan_out is the scan chain output. In normal system operation, signal scan_en is hold low, so system data $d l$ pass the multiplexer, scan_muxl, and goes into the D input of $d f f l$, while system data $d 2$ pass the multiplexer scan_mux2 and goes into the D input of $d f f 2$. In this mode, the scan inserted cir- 


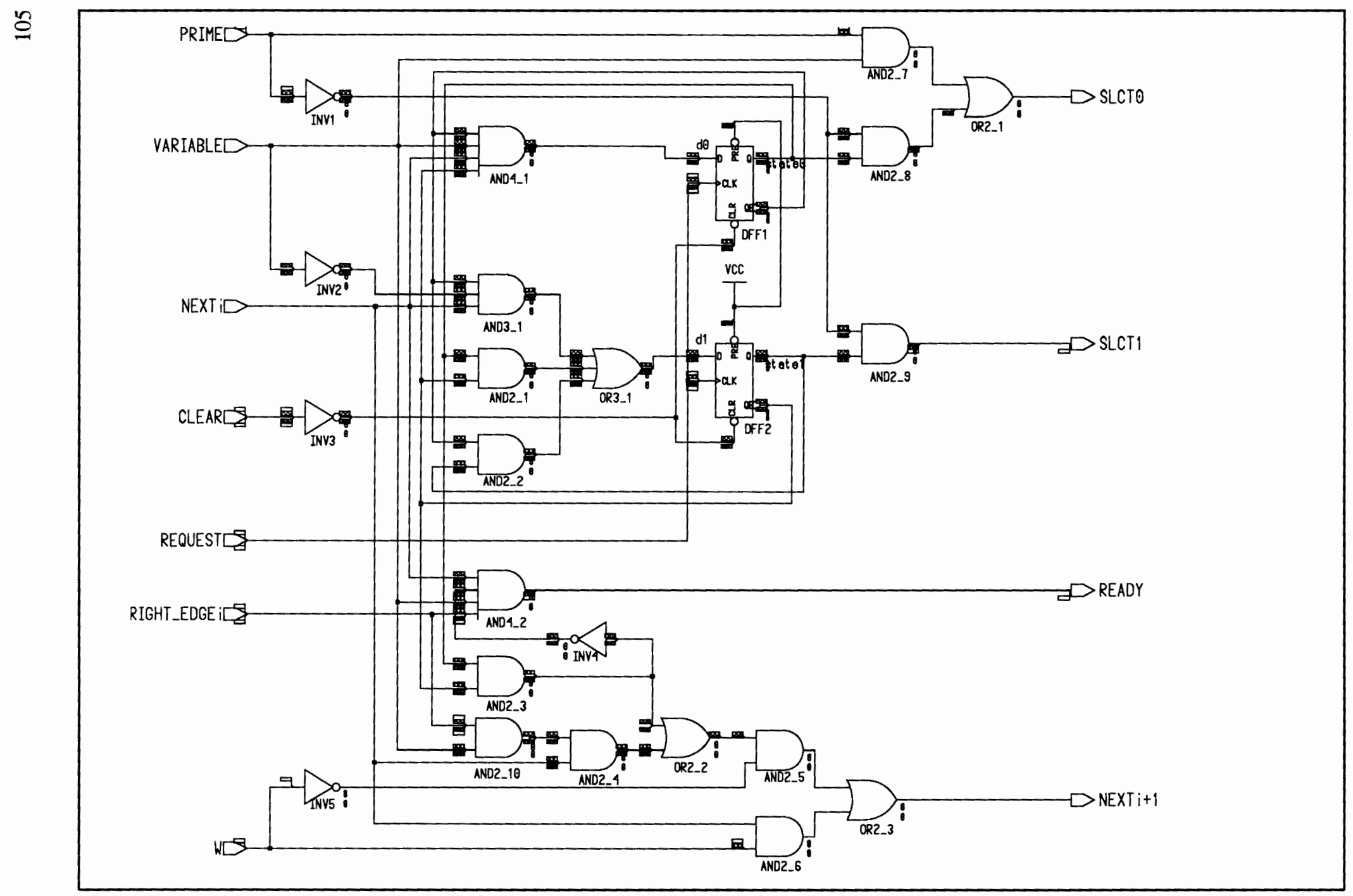

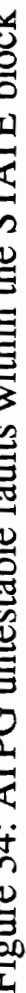


cuit behaviors exactly the same as the circuitry before scan insertion. The scan_en signal is hold high during the scan mode. In the scan mode, signal scan_in passes scan_muxl and goes into the data input of $d f f l$, the output from dffl, scan_outl (which is the same signal as $q 1$ ), passes the scan_mux2 and goes into the data input of $d f f 2$, the output of dff2 is the scan output, scan_out. This path is shown in Figure 36 in dark line. This path stitches $d f f l$ and $d f f 2$ together and makes it possible to load desired data to the outputs of the two D flip-flops. This way, the STATE block can behave like a combinational circuitry during ATPG process.

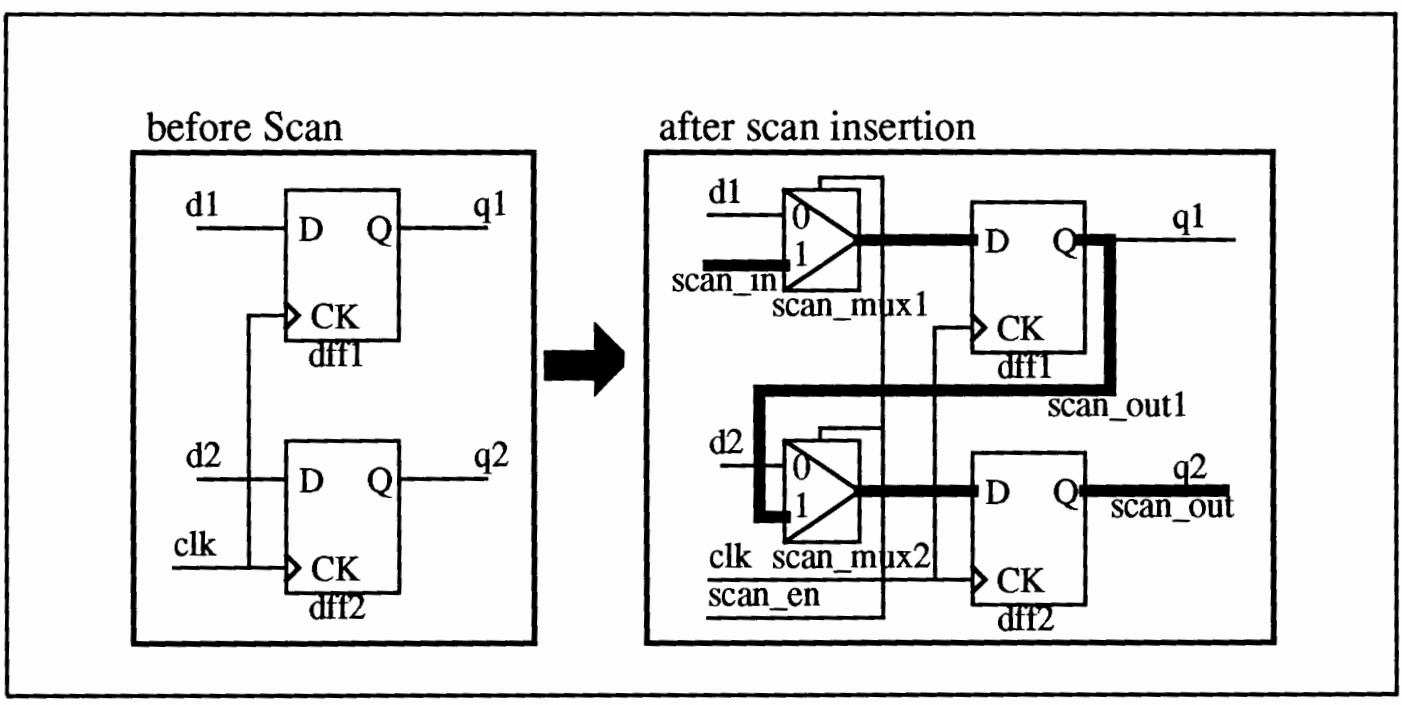

Figure 35: dffs in STATE block before and after scan insertion

The scan inserted STATE block is captured in STATE_SCAN.

To run ATPG on the scan inserted design, a test procedure file is needed to inform FastScan how to operate the scan chain. Figure 36 shows the content of the test procedure file.

This test procedure file has two procedures, shift and load_unload.

The shift procedure describes how to shift data one position down the scan chain, by toggling the clock, forcing the scan input (setting a desired value on the scan input), and strobing the scan output. The force_sci keyword defines the forcing of scan input. The measure_sco keyword defines the strobing of the scan output. The forcing of scan 
input and the measuring of scan output should happen at time 0 , then the scan shift clock REQUEST goes to high at time 1, the scan shift clock REQUEST returns back to low at time 2 to complete one shift of scan data.

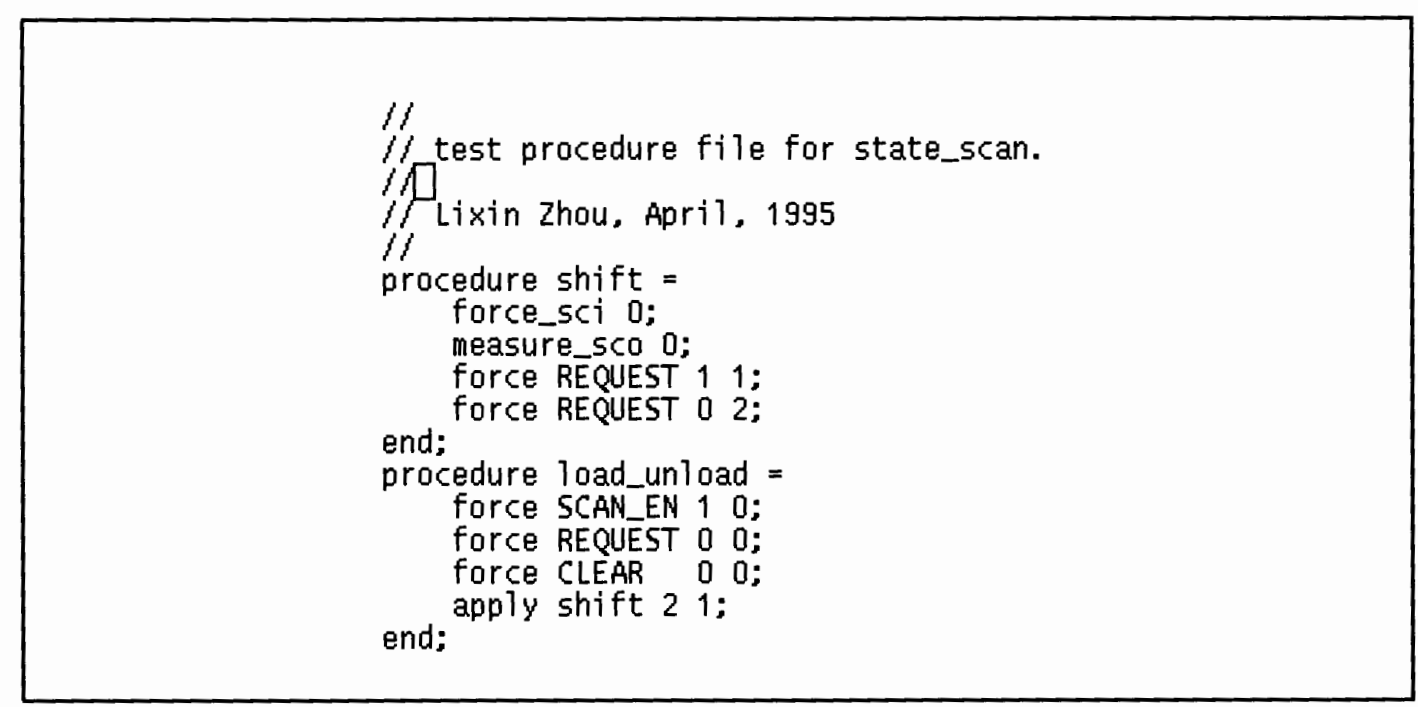

Figure 36: Test procedure file for the STATE_SCAN block

The load_unload procedure defines how to load and unload the scan chain in the scan group. For loading the scan chain, we must force the circuit into the appropriate state before the start of the shift sequence. This includes forcing the scan clocks, CLEAR and REQUEST, to their off-state, logic 0 , enabling the scan enable signal, scan_en, to put the circuitry into scan mode. Then the shift procedure can be applied. Since there are two memory elements in the scan chain, the shift procedure should be applied twice.

After invoking FastScan on the STATE_SCAN block, scan information need to be defined which includes defining the scan clocks and their off-states, defining scan group and its test procedure file, and defining the scan chain with its scan input and scan output. Details of the operations can be found in Appendix B.

The report file from FastScan is shown in Figure 37. The test coverage is now 100\%. The testability design on the STATE block was successful.

There are two tied faults (TI) in this block. This is shown in Figure38. Since the pre 
pins of these two flip-flops are tied to Vcc, stuck-at 1 faults on these two pins can not be detected, there are therefore classified as tied faults. These two stuck-at 1 faults will not cause any functionality failure of our design.

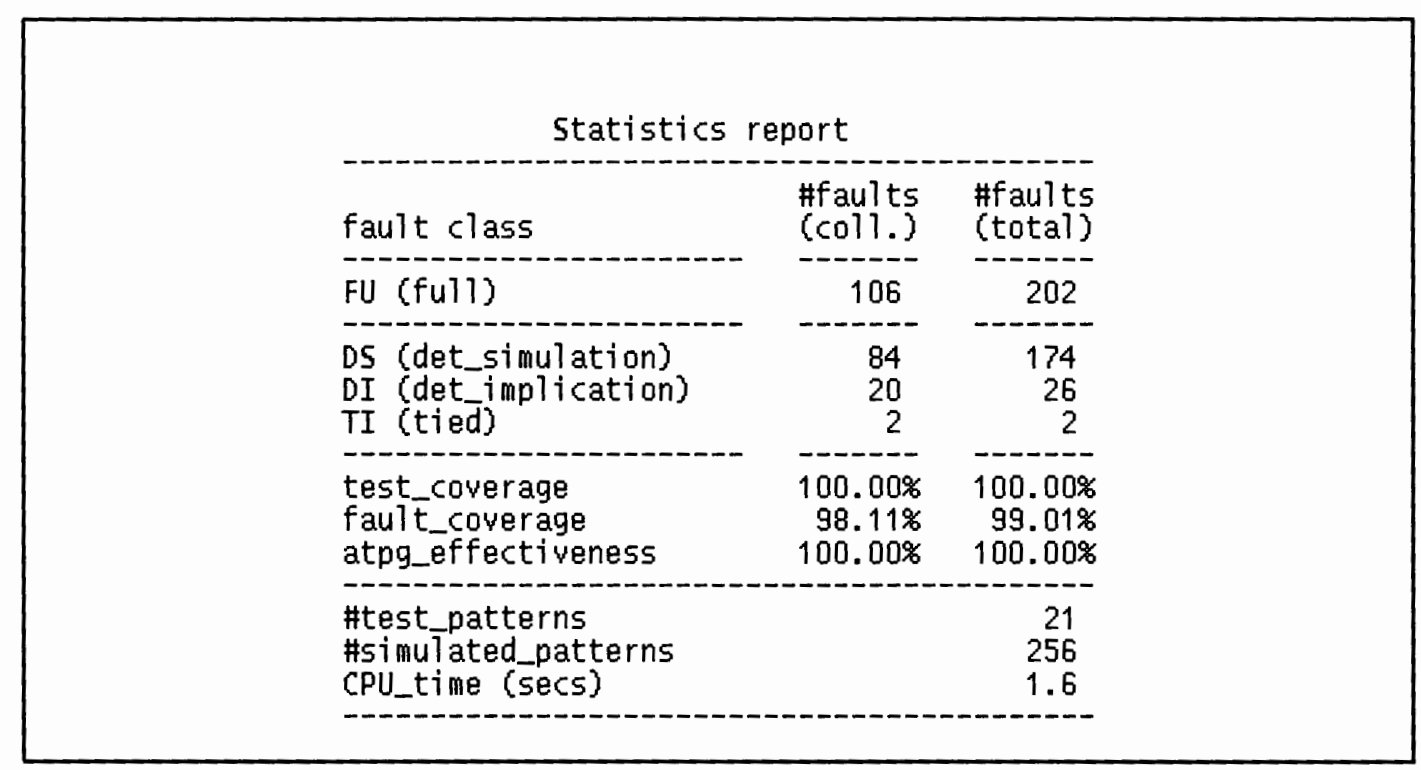

Figure 37: FastScan report file for STATE_SCAN block

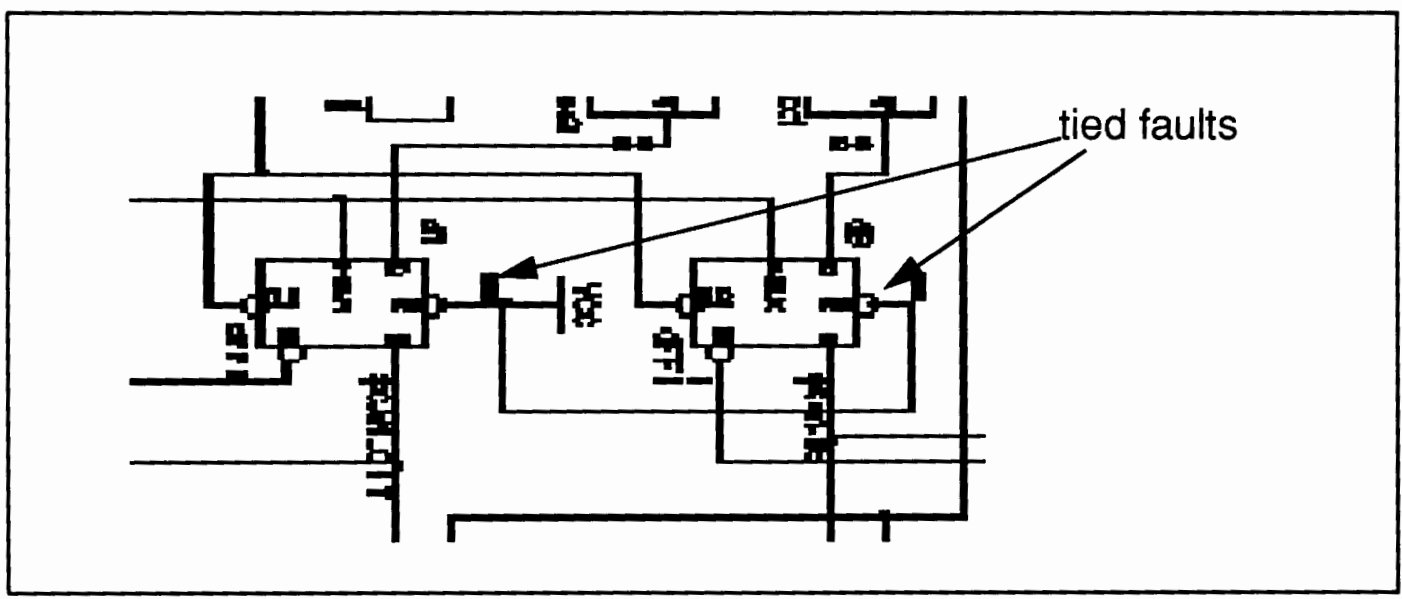

Figure 38: Tied faults in the STATE_SCAN block

DI in Figure 37 stands for the detected by implication faults. These faults are located on the scan chain which can be detected by loading and unloading the scan chain. 
Detected by implication faults in the STATE_SCAN block are shown in Figure 39.

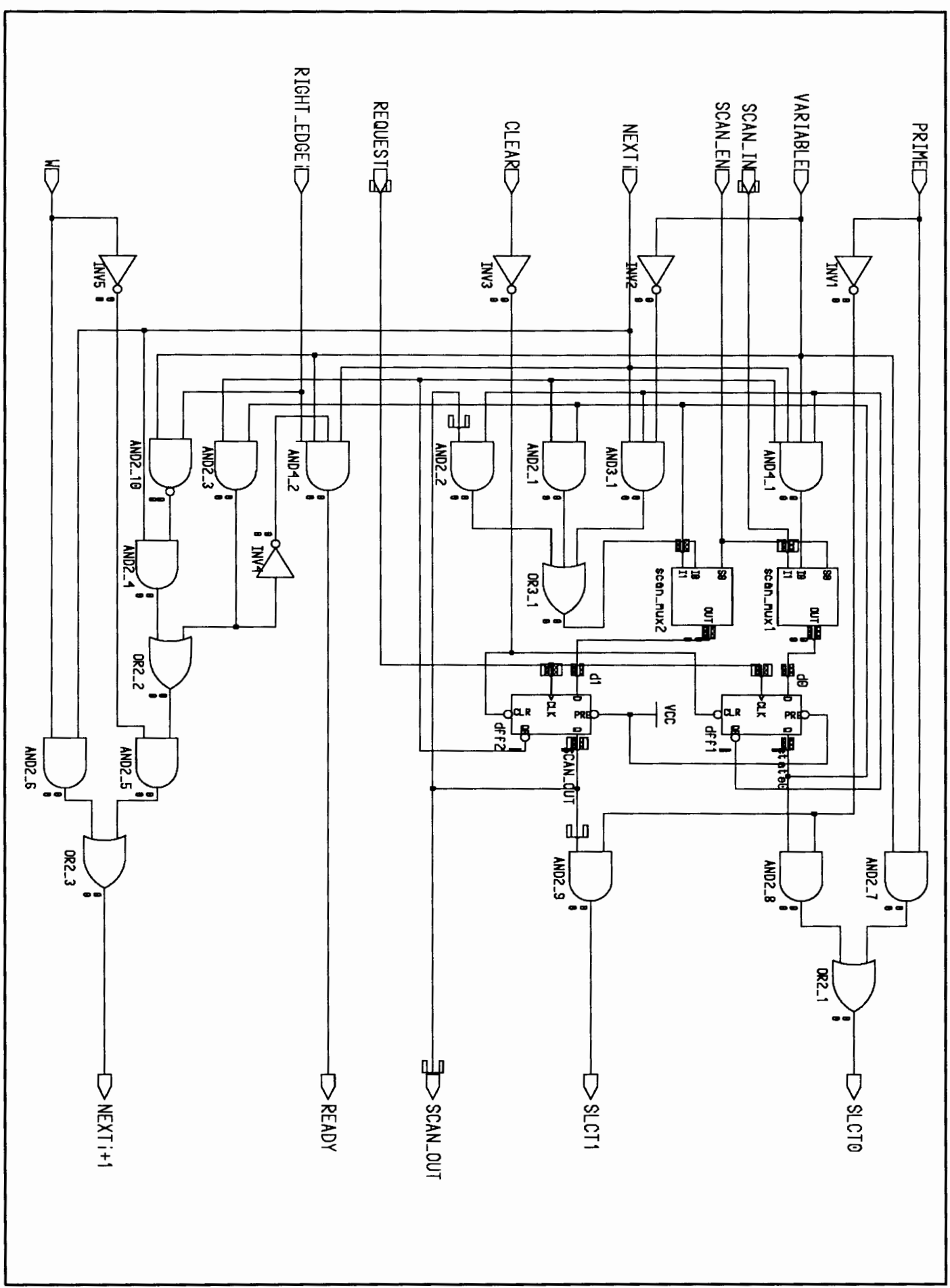

Figure 39: Detected by implication faults in the STATE_SCAN block 


\section{Testability Analysis of the IT block}

The IT block includes the STATE block, the COUNTER block, the IDENT block, and some combinational logic. Since the STATE block, the COUNTER block, and the IDENT block all had been identified as highly testable. A high test coverage on the IT block was expected.

Since the STATE_SCAN block is the only block within the IT block that has scan circuitry, the scan setup on the IT block is exactly the same as that of the STATE_SCAN block. The transcript of FastScan running on the IT block can be found in Appendix B.

Figure 40 shows the report file from FastScan for the IT block. The test coverage is 98.62\% before fault collapsing. There are eight faults identified as ATPG untestable faults in this block. Let's take a close look at these ATPG untestable faults to see whether they are a real problem or not.

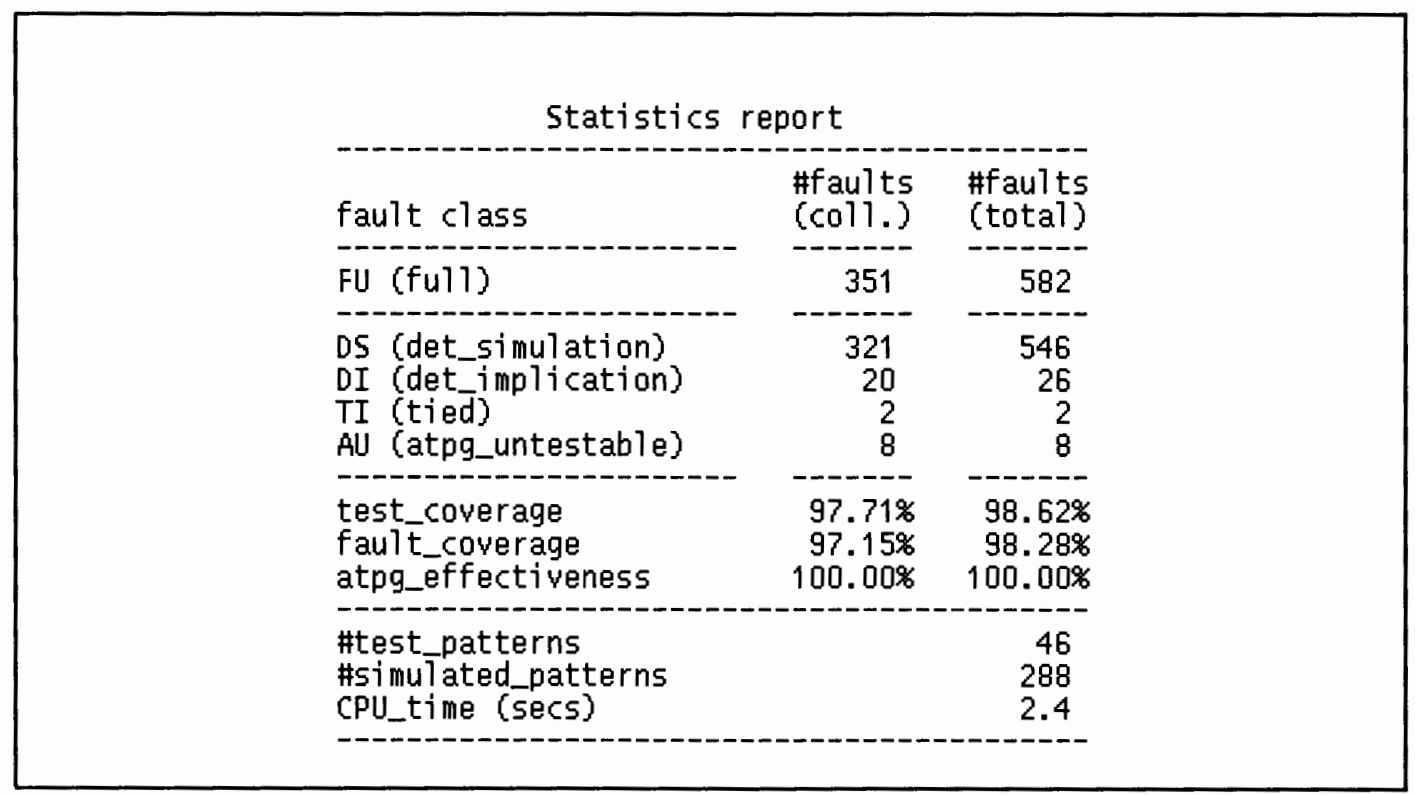

Figure 40: FastScan report file for the IT block

The ATPG untestable faults in the IT blocks occur on the undriven inputs of the four multiplexers. Figure 41 shows a pair of ATPG untestable faults on an undriven input pin (I3) of a multiplexer in the IT block. Both stuck-at 0 and stuck-at 1 were identified 
as ATPG untestable. Since these pins are not used anyway, these ATPG untestable faults are not a problem at all.

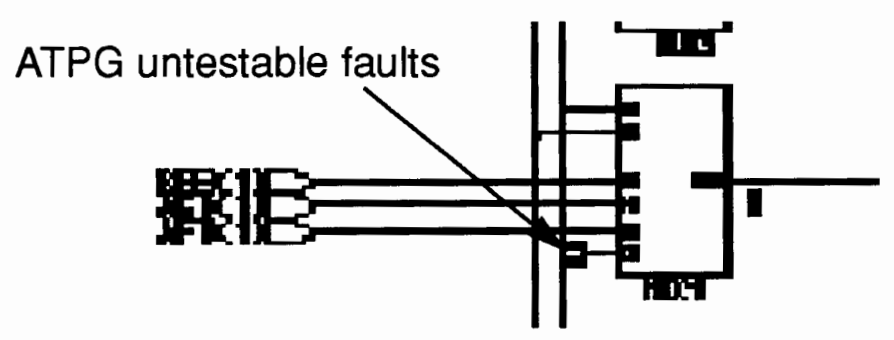

Figure 41: A pair of ATPG untestable faults on an undriven input pin (I3)

The two tied faults(TI) are from the STATE_SCAN block.

\section{Testability Analysis of the ILU}

The ILU has eight IT instances and some combinational logics. The scan chains from different IT instances were stitched together to form a scan chain that links all the memory elements in the ILU, thus desired data can be loaded to the outputs of any memory elements inside the ILU by exercising this scan chain.

The scan shifting clock, REQUEST, will be uncontrollable from the CCM chip input pins after the control unit is integrated with the ILU. This is because the REQUEST signal will be generated by the control unit. This will make the scan chain useless. To solve this problem, a multiplexer was added as shown in Figure 42. In normal system operation mode, the multiplexer select signal, TEST_MODE, will be hold low to pass the REQUEST signal from the control unit to the REQUEST input pins of the ITs. In testing mode, the TEST_MODE will be hold low to pass the TEST_CLK to shift the scan chain. Both the signals TEST_MODE and TEST_CLK should be directly accessible from the CCM chip (which means TEST_MODE and TEST_CLK should be CCM chip input pins). 


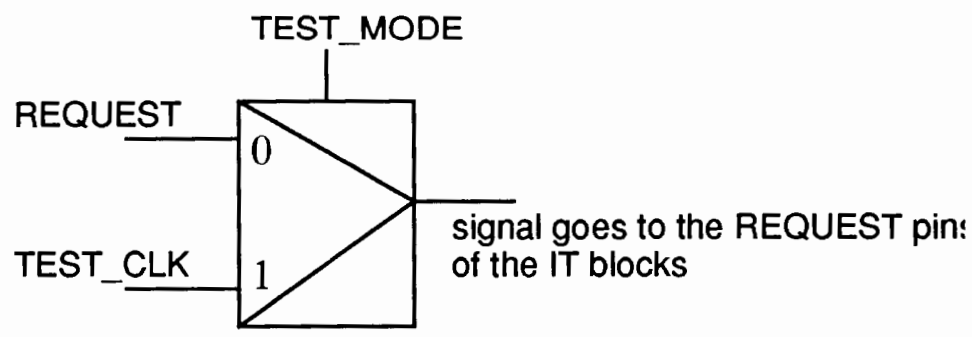

Figure 42: Illustration of the muxed REQUEST signal

The test procedure file for the scan chain in the ILU is shown in Figure 43. This test procedure is pretty much the same as the test procedure file used in the STATE_SCAN block. The differences are: the TEST_CLK signal is used instead of the REQUEST signal to shift the scan chain; the TEST_MODE signal should be hold high during the testing to pass through the TEST_CLK signal; and the shift procedure need to be applied 16 times since there are 16 state elements in the ILU.

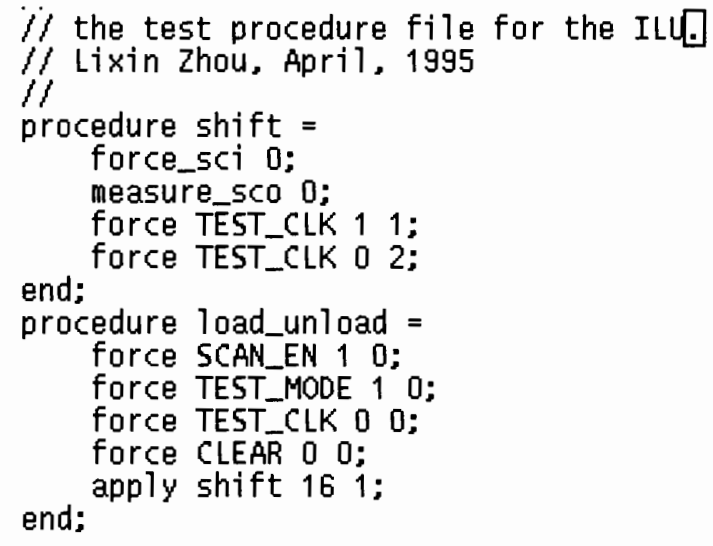

Figure 43: The test procedure file for the scan chain in the ILU

FastScan report file for the ILU is shown in Figure 44.

The test coverage for the ILU is $98.30 \%$ before faults collapsing. There are 70 ATPG 
untestable faults in the ILU. Since there are 8 ATPG untestable faults in each IT cell, 64 of them are from the eight IT cells in the ILU. Let's take a look at the other 6 ATPG untestable faults. Figure 45 shows the location of these 6 ATPG untestable faults. There is a stuck-at 1 ATPG untestable fault on the input pin TEST_MODE and a stuck-at 1 ATPG untestable fault on the select input of the TEST_MUX cell. During the testing, the TEST_MODE is pin constraint to a constant 1 , FastScan was not able to set a value of 0 at these two nodes, therefore these two faults were identified as ATPG untestable faults. Also because of the pin constraint on the TEST_MODE, the fault effects of both the stuck-at 1 and stuck-at 0 faults on the REQUEST pin and the I0 input pin of the TEST_MUX cell are not able to pass the TEST_MUX to the observe points, therefore they were identified as ATPG untestable faults. Although these six ATPG untestable faults can not be detected by the FastScan test patterns, they can be definitely detected by the functional testing on the CCM chip. Because when any of these faults happen, the clock signal for the state machines will be hold, the state machines in side the STATE blocks will not function at all.

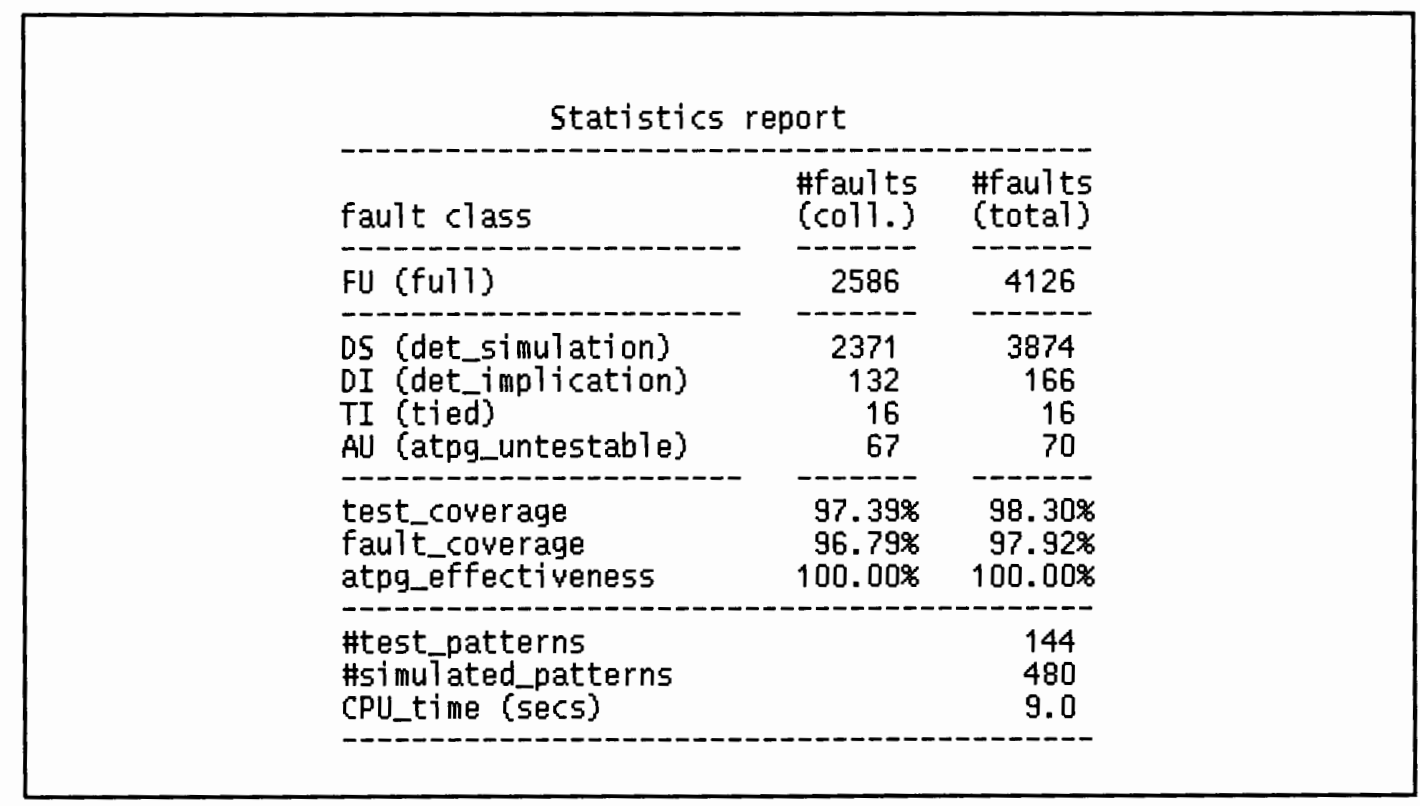

Figure 44: FastScan report file for the ILU 
Now, we can claim that all the single stuck-at fault in the ILU that might cause functional failures (remember, untestable faults do not cause functional failure) can be detected.

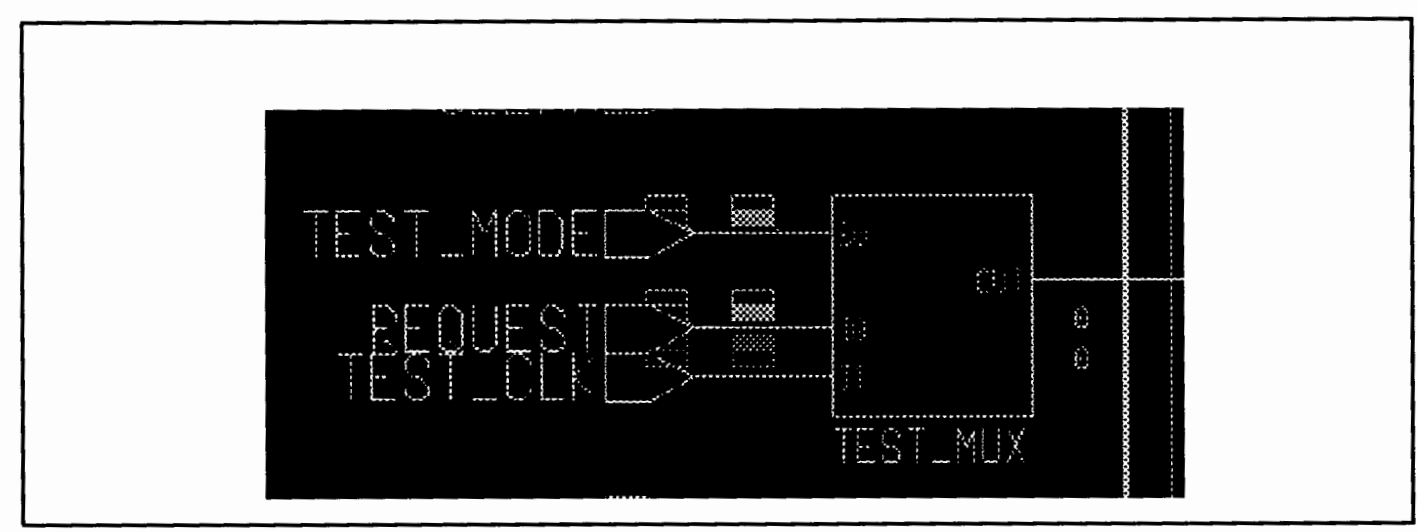

Figure 45: ATPG untestable faults in the ILU

A copy of test patterns for detecting single stuck-at faults in the ILU is located in the /u/mperkows/ccm on the EE computer system of Portland State University.

\subsubsection{Test Coverage Comparison}

It is interesting to compare the test coverage on the ILU with and without scan circuitry. FastScan report on ILU without scan design is shown in Figure 46. We can see that the test coverage of ILU jumps from $67.94 \%$ to $98.30 \%$ at the cost of 17 2-to-1 multiplexers (two 2-to-1 multiplexers in each IT cell plus the one 2-to-1 multiplexer which is used to select between the REQUEST signal and the TEST_CLK signal).

\subsubsection{An Overview of Testability Design and Analysis on the CCM}

This section discusses how to integrate a scan inserted ILU with a scan inserted control unit to obtain a highly testable CCM. 


\begin{tabular}{|c|c|c|}
\hline fault class & $\begin{array}{l}\text { \#faults } \\
\text { (colt.) }\end{array}$ & $\begin{array}{l}\text { \#faults } \\
\text { (total) }\end{array}$ \\
\hline FU $(f u] 1)$ & 2586 & 4126 \\
\hline $\begin{array}{l}\text { DS (det_simulation) } \\
\text { PU (posdet_untestable) } \\
\text { TI (tied) } \\
\text { AU (atpg_untestable) }\end{array}$ & $\begin{array}{r}1769 \\
9 \\
16 \\
792\end{array}$ & $\begin{array}{r}2780 \\
25 \\
16 \\
1305\end{array}$ \\
\hline $\begin{array}{l}\text { test_coverage } \\
\text { fault_coverage } \\
\text { atpg_effectiveness }\end{array}$ & $\begin{array}{r}69.01 \% \\
68.58 \% \\
100.00 \%\end{array}$ & $\begin{array}{r}67.94 \% \\
67.68 \% \\
100.00 \%\end{array}$ \\
\hline $\begin{array}{l}\text { \#test_patterns } \\
\text { \#simulated_patterns } \\
\text { CPU_time (secs) }\end{array}$ & & $\begin{array}{l}119 \\
480 \\
8.8\end{array}$ \\
\hline
\end{tabular}

Figure 46: FastScan report file on ILU without scan design

The control unit of the CCM is basically a state machine. It will have testability problems using FastScan without scan insertion. The mux-scan scan insertion method used in the STATE block can be applied in the testability design of the control unit. The integration of the scan inserted ILU and a scan inserted control unit is illustrated in Figure 47 (system signals are not shown in this graph). The scan chains inside the control unit and the ILU are stitched together to form one scan chain for the CCM as highlighted in the graph. When in test mode, signal test_mode and scan_en should be hold high, same scan shifting clock should be applied to both clk and the test_clk inputs of the CCM. With the testability design strategies described here, a highly testable CCM can be expected.

\subsection{Verification of the Design's Functionality after Scan Insertion}

It is important to reverify a design's functionality and timing after scan circuitry has been inserted. The inserted scan circuitry might have changed the design's functional- 


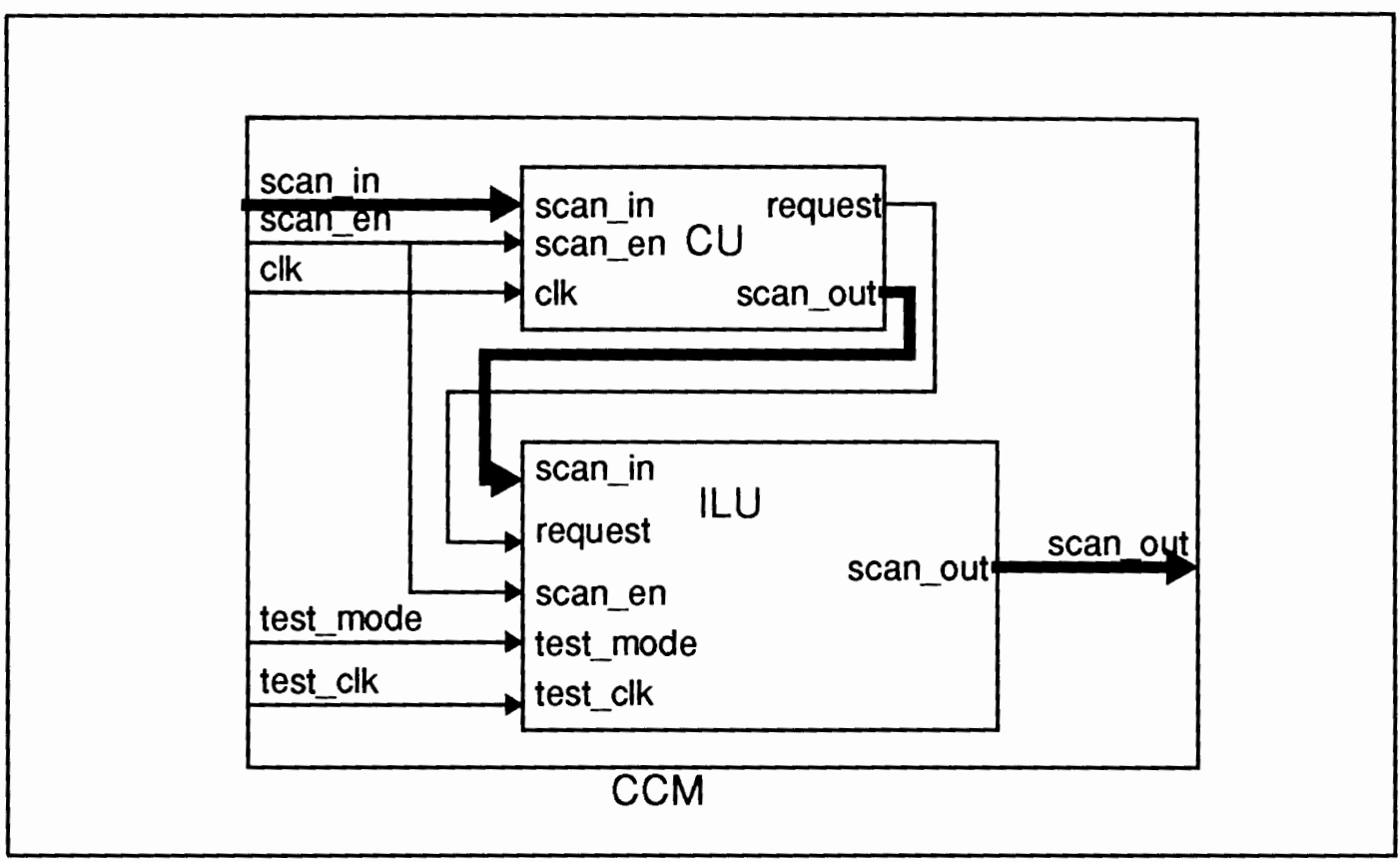

Figure 47: ILU and CU scan circuitry integration

ity unintentionally, especially when the scan circuitry was inserted manually. The inserted scan circuitry also might have introduced extra gate delay (like the scan_mux that was added in the STATE_SCAN block), added extra gate loading capacitance and wire delay of some critical paths in the design.

There are two ways to reverify the functionality. One is using the commercial available formal verification tools which formally compare the functionality of the design before and after the scan insertion. The other is to rerun the functional test vectors on the scan inserted design.

The only way to verify the timing is to rerun the functional test with timing and also use statistic timing analysis tools (like QuickPath from Mentor Graphics) to analyze the critical paths after the scan insertion.

In our case, since the timing files of the netlist primitives are not available, running functional test with timing can not be performed. It should be performed after a specific VLSI technology has been selected to manufacture the CCMs.

Functional test was performed on the scan inserted ILU. The test cases used were the same as those discussed in section 5.2. The scan inserted ILU passed the functional 
test successfully.

5.5 Generation of a VHDL Model for the ILU

A VHDL model of the scan inserted ILU was generated by using VHDLWriter from Mentor Graphics.

This VHDL model is a structural description of the ILU in VHDL format. It includes the structural descriptions of all the subcomponents used in the ILU. It can be used as a documentation of the ILU structure in VHDL. A copy of the VHDL model can be found in $/ \mathrm{u} / \mathrm{mperkows} / \mathrm{ccm} / \mathrm{VHDL}$ on the EE computer system of Portland State University. 


\section{CHAPTER VI}

\section{CONCLUSIONS AND FUTURE WORK}

A machine based on a new architecture in which the data path has been designed to execute operations of "cube calculus", an algebraic model popularly used in the processing and minimization of Boolean functions, is presented in this thesis.

This machine, CCM, can execute logic operations in high-level formal systems in a real-time fashion. The CCM targets applications in logic minimization, logic synthesis, pattern recognition, and image processing. It can be implemented as a co-processor to a host computer or as a hardware accelerator.

The first version of the CCM, CCM1, was proposed by Kida and Dr. Marek Perkowski. Designing a machine which can efficiently execute all cube calculus operations has been a project of Dr. Marek Perkowski's research group for years. The author of this thesis was actively involved in this project. His contributions to the CCM project are outlined below as a conclusion of this thesis.

- Jointly designed a CCM2, one version of the CCM described in this thesis, as a member of group in Dr. Marek Perkowski's Computer Architecture for Robotics and Artificial Intelligence courses. The CCM2 is a much improved version of the CCM1. CCM2 introduced the following new concepts to the logic machines: (1) Relations and operators can be arbitrary (programmable) functions of input variables. This allows us to create an extremely large number of logic operations by combining basic operators. (2) While CCM1 execute only set-theoretical operations on literals, CCM2 introduces simple and complex symbols, as an intermediate level between bits and variables. This allows to realize arbitrary truly multiple-valued logic and to deal with the literals being various kinds of numbers. CCM2 can 
also deal with data such as: number intervals, symbolic predicates, associative tuples, and multiple-valued multiple-output relations. These capabilities greatly expand its semantics and the range of potential applications. (3) The CCM2 machine is microprogrammed to make the best use of the above property. (4) CCM2 is a general-purpose computer. It belongs to a superset of standard arithmetic computers, string matching computers, associative processors and long-word computer. Mainly, the author designed the control block of the IT cell, the iterative signals between ITs, and part of the control unit.

- Designed testing circuitry for functionality testing of a FPGA based CCM.

- Captured, simulated the CCM2 using Mentor Graphics Electronic Design Automation tools. Since Mentor Graphics EDA tools are installed and supported in the EE computer system of Portland State University, learning, exercising, and expanding of the CCM will be much easier for other students. The QuickSim II simulation setups of various cube calculus operations have been created and saved in force files. By executing a cube calculus force file within QuickSim II, the lists and wave forms of the input and output signals of that cube calculus operation can be displayed on the screen automatically. This makes learning and verifying cube calculus operations much more intuitive.

- Proposed a novel testability design and testability analysis approach, the bottom-up approach. This approach makes the testability design and analysis of a design more efficient, especially for large designs. This bottom-up approach was used in the testability design and analysis of the CCM.

- Performed testability analysis and testability design of the ILU. Testability problems within the ILU were identified. Full-scan design was inserted into the original design. The test coverage of the ILU was increased from $67.94 \%$ to $98.30 \%$ at the cost of 17 2-to-1 multiplexers.

- Discussed the testability analysis and design method of the CCM (including the control unit and the ILU). Testability design methods and strategies of the control unit and the CCM were given. 
- Created a VHDL model of the scan inserted ILU.

- Wrote an introduction to basic test theory and design-for-test concepts. This introduction covers all the design-for-test concepts and strategies used in the testability design and analysis of the ILU. This makes this thesis self-sufficient for other students who intend to use this thesis as a reference to do the testability design and analysis of the CCM control unit.

The electronic design of the ILU and other related materials are saved in $/ \mathrm{u} /$ mperkows/ccm on the EE computer system of Portland State University.

Suggestions are given here for future work on the CCM design:

- Functionality design of the control unit.

- Testability design and analysis of the control unit. The testability design and analysis methods which can be used on the control unit are well explained in this thesis.

- Generating test vectors for the CCM. FastScan can be used to generate test vectors.

- Timing verification of the CCM by using timing files of the netlist primitives from an ASIC vendor.

- Physical design of the CCM and timing reverification. Physical design approach can be chosen from standard cell approach and full customer approach. 


\section{REFERENCES}

[1] “ASIC/IC Design-for-Test Process Guide," Mentor Graphics Corp., 1994

[2] B.J. Falkowski and Marek Perkowski, "Algorithm for The Generation of Disjoint Cubes for Completely and Incompletely Specified Boolean Functions," Int. Journal of Electronics, vol. 70, no. 3, 1991, pp.553-538

[3] David Foote "The Design, Realization and Testing of the ILU of the CCM2 Using FPGA Technology", Portland State University, 1994

[4] "DesignArchitect User’s Manual," Mentor Graphics Corp., 1994

[5] Engelbart, C., "The Multiple-Valued Cube Calculus Machine Version 2.5," Department of Electrical Engineering, Portland State University, August 1993

[6] Edward J. McCluskey, "Logic Design Principles -- with an emphasis on testable semicustom circuits", Prentice-Hall, 1986

[7] "FastScan Reference Manual," Mentor Graphics Corp., 1994

[8] Frank F. Tsui, "LSI/VLSI Testability Design", McGraw-Hill, 1986

[9] H. Fuijiwara and T. Shimono, "On the acceleration of test generation algorithms," IEEE Transitions on Computers, vol. c-32, no. 12, Dec. 1983, pp 1137-1144

[10] Ibid. 
[11] J. Max Cortner, "Digital Test Engineering”, John Wiley \& Sons, 1984

[12] J. P.Roth, "Diagnosis of automata failures: a calculus and a methods", IBM Journal of Research and Development, vol. 10, Jul. 1966, pp.278-291

[13] L.P., Kida and Marek Perkowski, "The Cube Calculus Machine: A Ring of Asynchronous Automata to Process Multiple-Valued Boolean Functions," Portland State University, 1990

[14] Lawrence H. Goldstein and Evelyn L. Thigpen, "SCOAP: Sandia controllability/observability analysis program," Proceedings of the 17th Design Automation Conference, Jun. 1980, pp. 190-196.

[15] Marek Perkowski and J. Brandenburg, "Solving Basic Boolean Algebra Problems on a Hypercube Computer," Portland State University

[16] Marek Perkowski, "Systolic Architecture for the Logic Design Machine," Portland State University, 1985

[17] Marek Perkowski, M. Helliwell, and P. Wu, "Minimization of Multiple-Valued Input Multiple-Valued output Mixed-Radix Exclusive Sums of Products for Impletely Specified Boolean Functions," Proc. 25th ACM/IEEE Design Automation Conf., pp. 427-432, June, 1989

[18] Marek Perkowski, P. Dysko, and B.J. Falkowski, "Two Learning Methods for a Tree-Search Combinatiorial OPtimizer," Proc. IEEE, 1990, pp. 606-613

[19] Marek Perkowski, "A Synergistic Approach to Logis, Design, Computers and Problem-Solving," Ch. 22, 1991 
[20] Marek Perkowski, "The Cube Calculus Machine Number Three," Portland State University, 1991

[21] Neil H. E. Weste and Kamran Eshraghian, "Principles of CMOS VLDI Design - a system perspective", Addison-Wesley, second edition

[22] Prabhakar Goel, "An implicit enumeration algorithm to generate tests for combinational logic circuits," IEEE Transactions on Computers, vol. c-30, no. 3, Mar. 1981, pp. 215-222 (P17)

[23] Prabhakar Goel and Barry C. Rosales, "PODEM-X - an automatic test generation system for VLSI logic structures," IEEE Proceedings of the 18th Design Automation Conference, Jun. 1981, pp. 260-268.

[24] “QuickSim II User’s Manual,” Mentor Graphics Corp., 1994

[25] R.G. Bennetts, "Design of Testbale Logic Circuits", Addison-Wesley, 1984

[26]Sasao, T, "HART: A Hardwarefor Logic Minimization and Verification", Proc. ICCD'85, Oct. 7-10, 1985.

[27] Srimat T. Chakradhar, Michael L. Bushnell, and Vishwani D. Agrawal, "Toward massively parallel automatic test generation," IEEE Transactions on CAD, vol. 9, no. 9, Sept. 1990, pp. 981-994. (P17 ATPG)

[28] Svoboda, A., "Boolean Analyzer", Proc. Information Processing 68, Amsterdam, North-Holland, 1969. 
[29] Svoboda A., "Parallel Processing in Boolean Algebra", IEEE TC, Vol. C-22, No. 9, pp. 848-851, Sept 1973.

[30] Ulug, Proc. IEEE Intern. Conference on Computers and Communications, Arizona, 1985.

[31] “VHDLWrite User’s Manual”, Mentor Graphics Corp., 1994

[32] Wah, B.W., Ma, Y.W.E., "MANIP - A Multicomputer Architecture for Solving Combinatorial Extremum-Search Problems", IEEE TC, Vol. C-33, No. 5, pp. 377 390, May 1984.

[33] W. Golumb, Shift Register Sequences, Revised Edition, Laguna Hills, Calif.: Aegean Park Press, 1982

[34] Yasura, H., Tsujimoto, T., Tamaru, K., "Parallel Exhaustive Search For Several NP-Complete Problems Using Content Addressable Memories", Proc. IEEE Intern. Conference on Circuits and Systems, ISCAS'88, pp. 333- 336. 


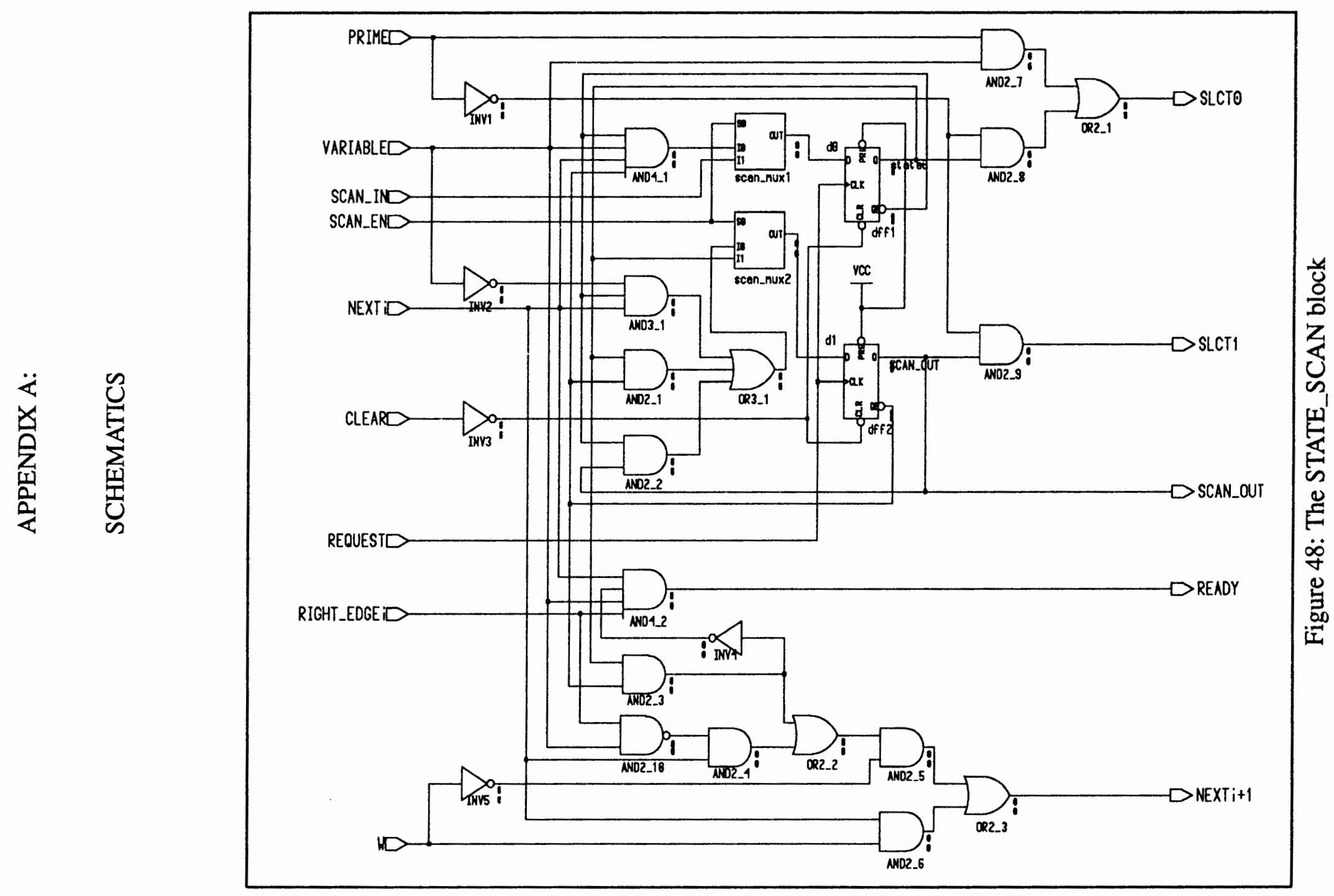




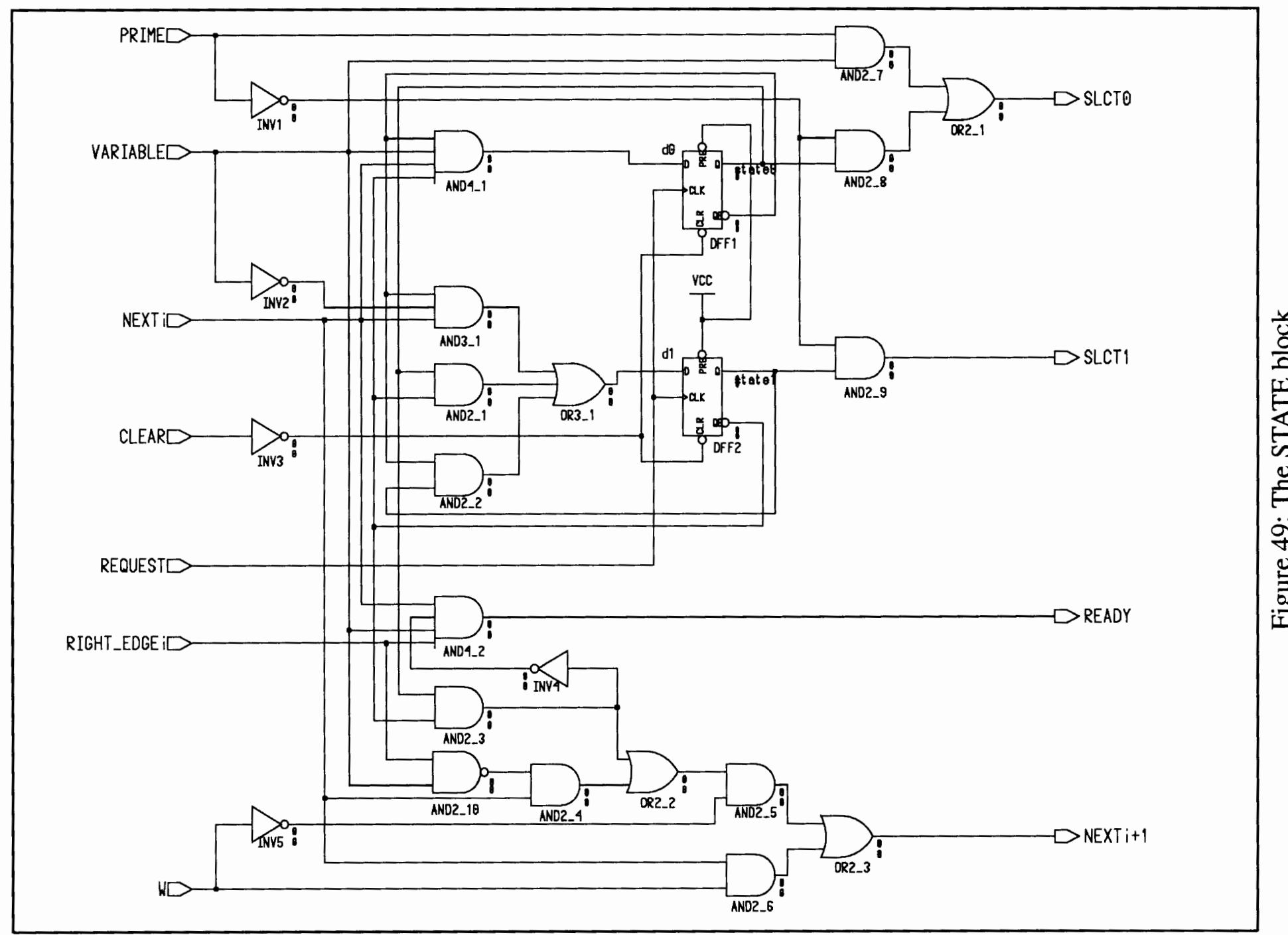




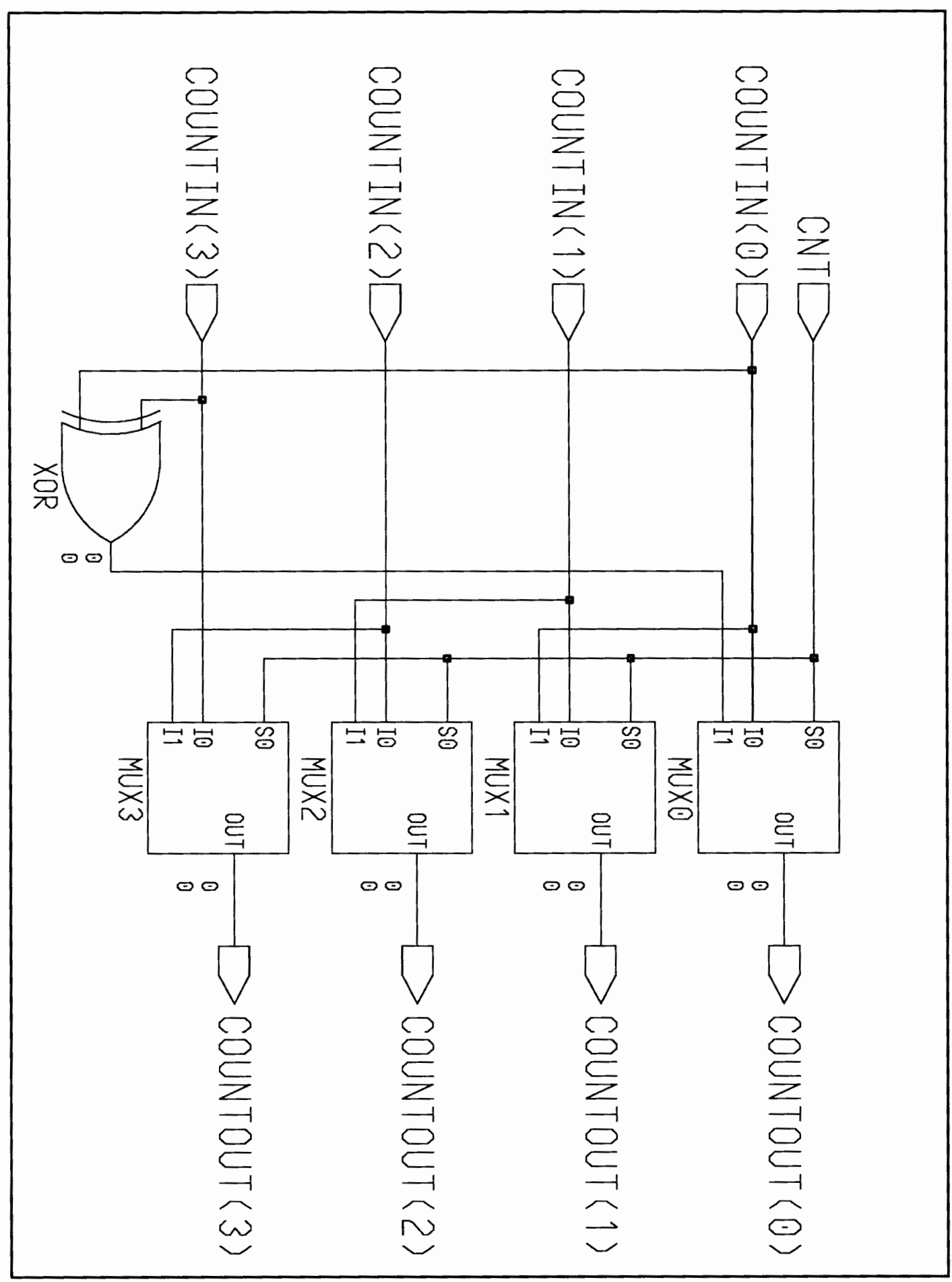

Figure 50: The COUNTER block 


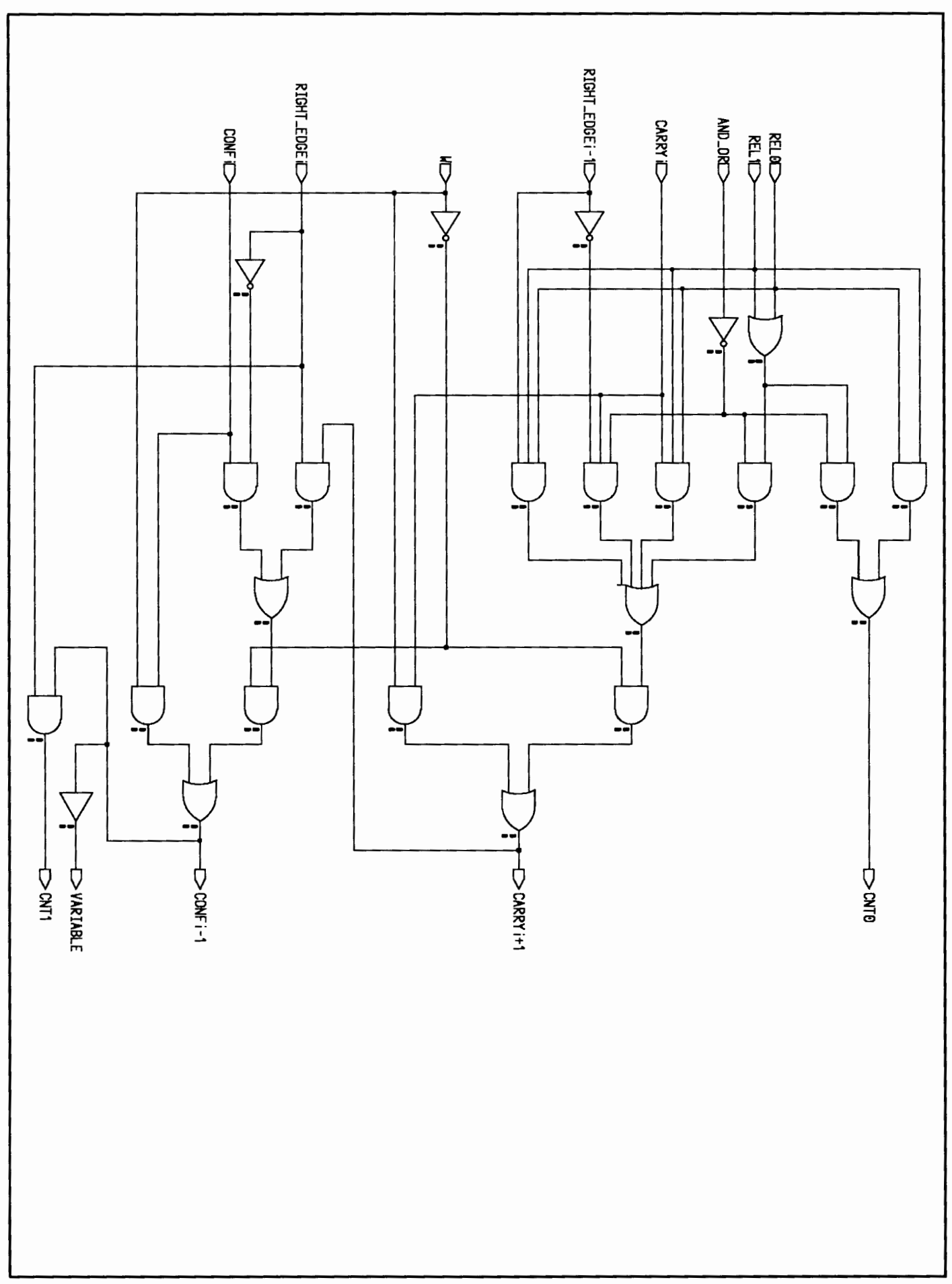

Figure 51: The IDENT block 


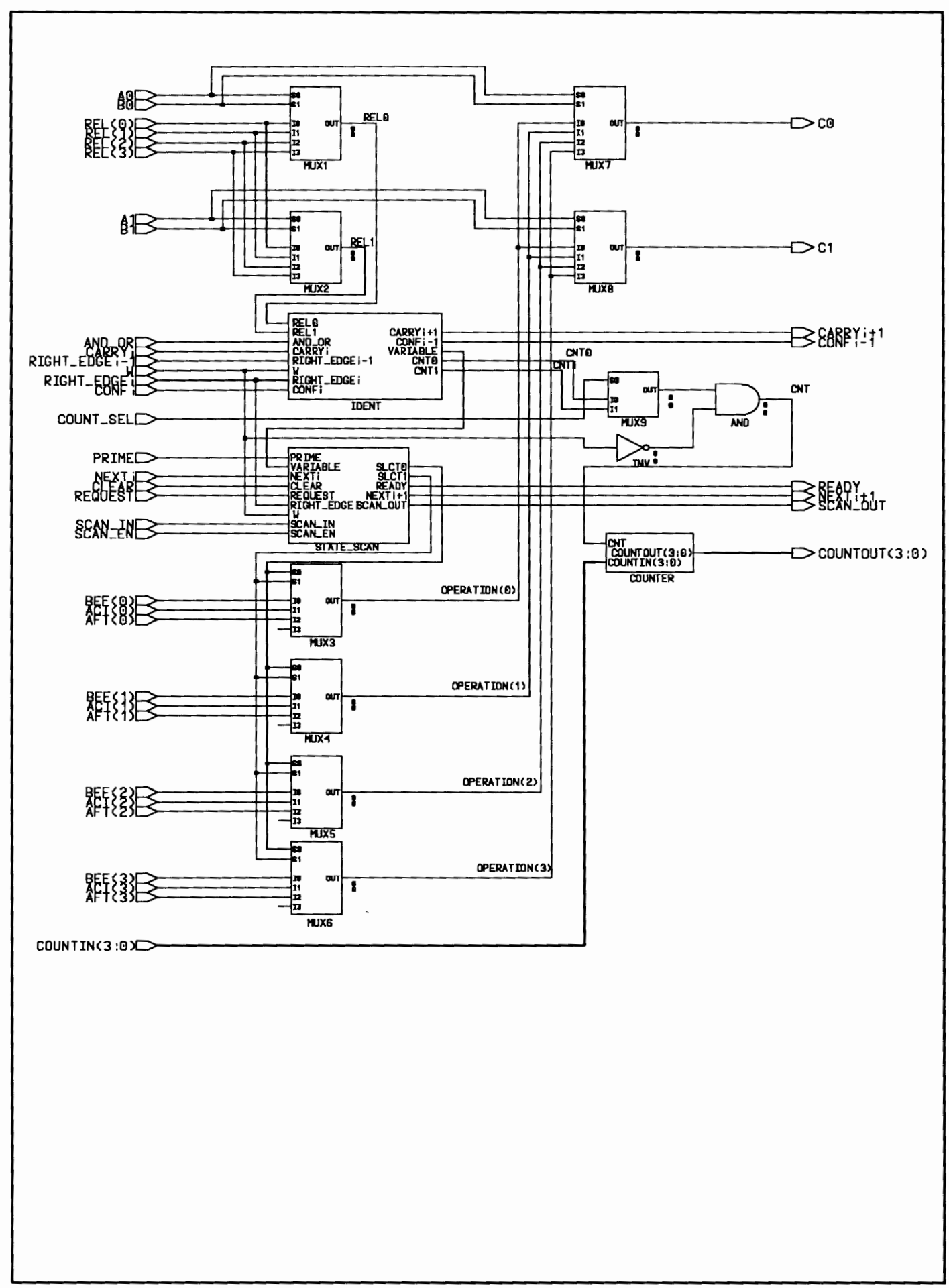

Figure 52: The IT cell 


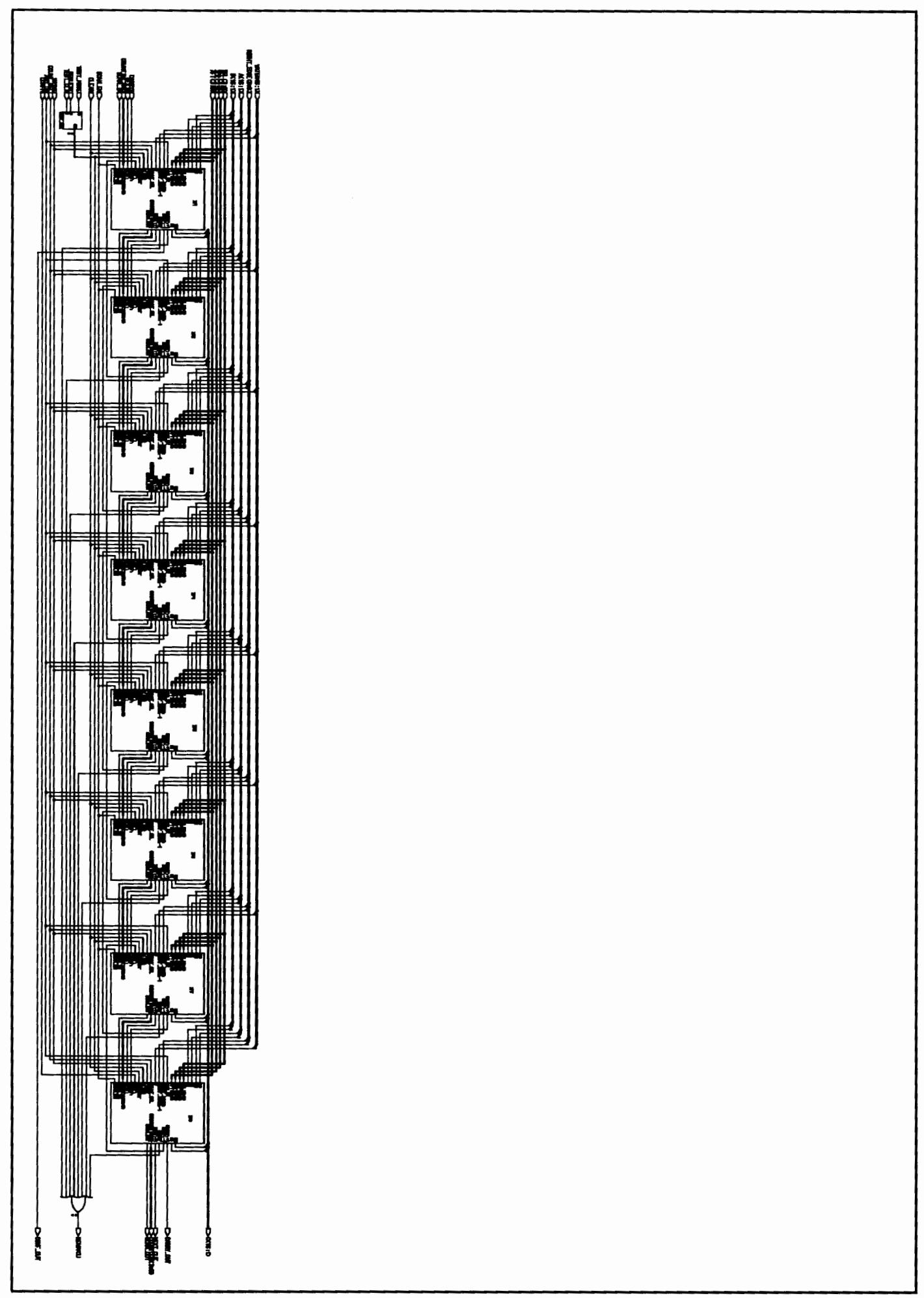

Figure 53: The ILU 


\section{APPENDIX B}

\section{FASTSCAN LOG FILES}

15 sunburnx fastscan countër -lib dft_stuff/genlib.atglib -nogui

/1 Fastscan v8.4_1.17 Fri Sep 30 23:09:40 PDT 1994

If.

$1 /$

II

/f Mentor Craphics software executing under Sun SPARC SunOS.

If Compiling library ...

/f Reading EDDM ...

SETUP> set system mode ATPG

// Flattening netlist ...

/f Flattening process completed, user_gates $=4$ sin_gates $=14$ PIs=5 POS $=4$.

// -

// Begin circuit learning analyses.

// Equivalent gates=0 classes=0 CPU time $=0.00 \mathrm{sec}$.

if Learning completed, implications $=0$, tied_gates $=0$. CPU time $=0.00 \mathrm{sec}$.

$1 /$ - -

/f Begin scan chain identification process, nemory elements $=0$.

/f circuit has no memory elements.

ATPG) add faults -all

ATPG> run

// Simulation performed for \#gates $=14$ \#fauits $=56$

1/ system mode $=$ ATPG pattern source = internal patterns

If -

If \#patterns test \#fauits \#faults \# eff. \#test process

/1 simulated coverage in list detected patterns patterns CPU time

// begin random patterns: capture clock $=$ none, observe point $=$ REC.PO
$1 / \quad 32$
$96.43 \%$
$100.00 \%$
$\begin{array}{rr}2 & 54 \\ 0 & 2\end{array}$
$54 \quad 6$
$\begin{array}{ll}6 & 6 \\ 2 & 8\end{array}$
$0.00 \mathrm{sec}$

ATPG> report statistics

Statistics report

\begin{tabular}{lcc} 
fault class & $\begin{array}{c}\text { \#faults } \\
\text { (coll.) }\end{array}$ & $\begin{array}{c}\text { \#faults } \\
\text { (total) }\end{array}$ \\
\hline FU (full) & 46 & 56 \\
\hline DS (det_simulation) & 46 & 56 \\
\hline test_coverage & $-100.00 \%$ & $100.00 \%$ \\
fault_coverage & $100.00 \%$ & $100.00 \%$ \\
atpg_effectiveness & $100.00 \%$ & $100.00 \%$ \\
\hline \#test_patterns & & 8 \\
\#Simulated_patterns & & 96 \\
CPU_time(secs) & & 1.2 \\
\hline
\end{tabular}

ATPG> save patterns results/counter.patterns ATPC> exit

Figure 54: the COUNTER block log file 


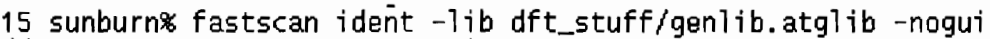

1/ FastScan v8.4_1.17 Fri Sep 30 23:09:40 PDT 1994

// Falcon Framework v8.4_1.1 wed May 25 17:42:40 PDT 1994

$1 /$ Mentor Graphics software executing under Sun SPARC Sunos.

// Compiling library ...

/1 Reading EDDM ...

SETUP> set system mode ATPG

1/ Flattening net7ist ...

1) Flattening process completed, user_gates $=0$ sim_gates $=37$ PIs $=8$ P0s $=5$.

$1 /$ - -

1) Begin circuit learning analyses.

1/ Equi valent gates $=2$ classes $=1$ CPJ time $=0.00$ sec.

1/ Learned gate functions: \#MUXs=3

1) Learning completed, implications $=21$, tied_gates $=0$, CPU time $=0.00 \mathrm{sec}$.

l) -

1/ Begin scan chain identification process, memory elements $=0$.

/1) Circuit has no memory elements.

ATPC $>$ add faut ts -all

ATPG $>$ run

$1 /$ simulation performed for \#gates $=37$ \#faults $=170$

(1) system mode $=$ ATPC pattern source = internal patterns

$1 /$ - 1 -

$1 /$ \#patterns test \#faults \#faults \# eff. \# test process

// simulated coverage in list detected patterns patterns CPU time

/) begin random patterns: capture clock = none, observe point $=R E C_{-} P O$

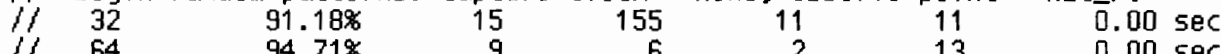

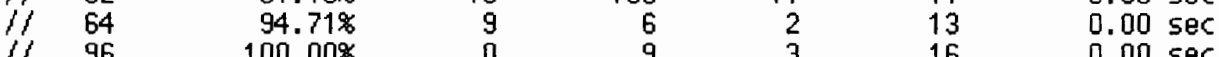

$16 \quad 0.00 \mathrm{sec}$

ATPG $>$ report statistics

Statistics report

\begin{tabular}{lcc} 
fault class & $\begin{array}{c}\text { \#faults } \\
\text { [col1.) }\end{array}$ & $\begin{array}{c}\# f a u l t s \\
\text { (total) }\end{array}$ \\
\hline FU (full) & 77 & 170 \\
\hline DS (det_simulation) & 77 & 170 \\
\hline test_coverage & $100.00 \%$ & $100.00 \%$ \\
fault_coverage & $100.00 \%$ & $100.00 \%$ \\
atpg_effectiveness & $100.00 \%$ & $100.00 \%$ \\
\hline \#test_patterns & & 16 \\
\#simulated_patterns & & 128 \\
CPU_time (secs) & & 1.3 \\
\hline
\end{tabular}

ATPC> saye patterns results/ident.patterns ATPC $>$ exit

Figure 55: the IDENT block log file 
i5 sunburno fastscan state - lib dft_stuff/genlib.atglib -nogui

// Fastscan v8.4_1.17 Fri Sep 30 23:09:40 PDT 1994

1/ Falcon Framewark v8.4_1.1 wed May 25 17:42:40 PDT 1994

// Mentor Graphics software executing under Sun SPARC Sunos.

// Compiling Tibrary ...

1/ Reading EDDM ...

SETUP> set system mode ATPC

// Flattening netlist ...

/) Flattening process completed, user_gates $=2$ sim_gates $=44$ PIs $=7$ pos $=4$.

$7 /$ - -

// Begin circuit learning analyses.

If Equivalent gates $=2$ classes $=1$ CPU time $=0.00$ sec.

// Learned gate functions: \#MUXs=2

1/ Learning completed, implications $=17$, tied_gates $=3$, CPU time $=0.00 \mathrm{sec}$.

/) Begin scan chain identification process, memory elements $=2$.

// - -

1) Warning: 2 non-scan memory elements converted to TIE-X gates. (DS)

li --no

1/ Begin scan clack rules checking.

if 0 scan clock/set/reset lines have been identified.

ATPG $>$ add faults -all

ATPG) run

l/ -

(1) Simulation performed for \#gates $=44$ \#faults $=50$

(1) system mode $=$ ATPG pattern source = internal patterns

If \#patterns test \#faults \#faults \# eff

(1) simulated coverage in list detected patterns patterns cPu time

$/ /$ begin random patterns: capture ciock $=$ none, observe point $=$ REG_PO
$1 / 32$ $32 \quad 24.44 \%$
9 with abort limit $=30$

oint $=$ REG_PO

(1) deter

ATPG) report statistics

with abort limit $=3$

0.00 sec

Statistics report

\begin{tabular}{|c|c|c|}
\hline fault class & $\begin{array}{l}\text { \#faults } \\
\text { (coll.) }\end{array}$ & $\begin{array}{l}\text { \#faults } \\
\text { (total) }\end{array}$ \\
\hline FU $(f u l l)$ & 90 & 180 \\
\hline $\begin{array}{l}\text { DS (det_simulation) } \\
\text { PU (posdet_untestable) } \\
\text { TI (tied) } \\
\text { AU (atpg_untestable) }\end{array}$ & $\begin{array}{r}15 \\
3 \\
2 \\
70\end{array}$ & $\begin{array}{r}2 \\
132\end{array}$ \\
\hline $\begin{array}{l}\text { test_coverage } \\
\text { fault_coverage } \\
\text { atpg_effectiveness }\end{array}$ & $\begin{array}{r}18.75 \% \\
18.33 \% \\
100.00 \%\end{array}$ & $\begin{array}{r}24.44 \% \\
24.17 \% \\
100.00 \%\end{array}$ \\
\hline $\begin{array}{l}\text { \#test_patterns } \\
\text { \#simulated_patterns } \\
\text { CPU_time (secs) }\end{array}$ & & $\begin{array}{r}64 \\
1.4\end{array}$ \\
\hline
\end{tabular}

ATPG) save patterns results/state.patterns ATPC) exit

Figure 56: the STATE block log file 
15 sunburn\% fastscan state_scan -lib dft_stuff/genlib.atglib -nogui

1/ Reading EDDM ...

SETUP> dofile dft_stuff/state_scan.do

/1 command: add clocks 0 ciear request

// command: add scan group g1 dft_stuff/state_scan.g1

1/ command: add scan chain c1 g1 scan_in scan_out

// command: set system mode ATPG

// Flattening netilist ...

1) Flattening process completed, user_gates $=4$ sim_gates $=49$ PIs $=9$ POS $=5$.

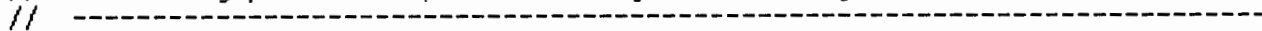

i) Begin circuit learning analyses.

$1 /$ Equivalent gates $=2$ classes $=1$ cPu time=0.00 sec.

if Learned gate functions: \#MUXs=2

1/ Learning completed, implications=17, tied_gates=3, CPU time=0.00 sec.

l)

// Begin scan chain identification process, memory elements $=2$.

/f Reading group test procedure file dft stuff/state scan.a1.

/f Simulating load/unload procedure in gi test procedure file.

If Chain $=c 1$ successfully traced with scan_cel1s $=2$.

/) Begin scan clock rules checking.

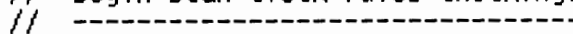

1/ 2 scan clock/set/reset lines have been identified.

// All scan clocks successfully passed off-state check.

/1 All scan clocks successfully passed capture ability check.

// Capture clock is set to REQUEST.

/) command: add faults -ali

1/ command: run

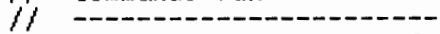

$1 /$ Simulation performed for \#gates $=49$ \#faults $=174$

1) system mode $=$ ATPG pattern source = internal patterns

$1 /$ - $1 /$ -

$1 /$ \#patterns test \#faults \#faults \# eff. \# test process

$1 /$ simulated coverage in 7ist detected patterns patterns cPu time

$1 /$ begin random patterns: capture clock = /REQUEST, abserve point = MASTER

$1 /$ begin random patterns: capture clock = /CLEAR, observe point = MASTER

1/ begin random patterns: capture clock $=$ none, observe point $=$ MASTER

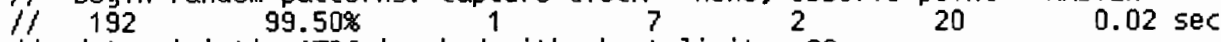

$1 /$ deterministic ATPC invoked with abort limit $=30$

// command: report statistics

statistics report

\begin{tabular}{lcc} 
fault class & $\begin{array}{c}\text { \#faults } \\
\text { (coll.) }\end{array}$ & $\begin{array}{c}\text { \#faults } \\
\text { (total) }\end{array}$ \\
\hline FU (ful1) & 106 & 202 \\
\hline DS (det_simulation) & 84 & 174 \\
DI (det_implication) & 20 & 26 \\
TI (tied) & 2 & 2 \\
\hline test_coverage & $-00.00 \%$ & $100.00 \%$ \\
fault_coverage & $98.11 \%$ & $99.01 \%$ \\
atpg_effectiveness & $100.00 \%$ & $100.00 \%$ \\
\hline \#test_patterns & & 21 \\
\#simulated_patterns & & 256 \\
CPU_time (secs) & & 1.6
\end{tabular}

ATPG > save patterns results/state_scan.patterns

ATPG> exit

Figure 57: the STATE_SCAN block log file 
15 sunburnx fastscān it_scän -lib dft_stuff/genlib.atglib -nogui

SETUP > dofile dft_stuff/it_scan. do

if command: add clocks 0 clear request

/1 command: add scan group g1 dft_stuff/state_scan.g1

1) command: add scan chain c1 g1 scan_in scan_out

1/ command: set system mode ATPC

// Flattening netlist ...

i) Flattening process completed, user_gates=9 sim_gates=191 PIs=37 pOs=11.

l/ -1/

if Begin circuit learning analyses.

I) Equivalent gates $=6$ classes $=3$ cPU time $=0.00 \mathrm{sec}$.

// Learned gate functions: \#MUXS=5

/) Learning completed, implications=46, tied_gates $=3$, CPU time=0.02 sec.

/f Begin scan chain identification process, memory elements $=2$.

// Reading group test procedure file dft_stuff/state_scan. 91 .

i) Simulating load/unload procedure in gi test procedure file.

I) Chain $=c 1$ successfully traced with scan_cells $=2$.

f) Begin scan clock rules checking.

(1)

1/ 2 scan clock/set/reset lines have been identified.

if All scan clocks successfully passed off-state check.

i) All scan clocks successfully passed capture ability check.

/) Capture clock is set to REQUEST.

/) command: add faults -all

l) command: run

If Simulation performed for \#gates = 191 \#faults $=546$

1) system mode = ATPG pattern source = internal patterns

// \#patterns test \#faults \#faults \# eff. \#tast process

i) simulated coverage in list detected patterns patterns CPU time

// begin random patterns: capture clock = /REQUEST, obserye point = MASTER

/) begin random patterns: capture clock $=/$ CLEAR, observe point = MASTER

1) begin random patterns: capture clock = none, observe point = MASTER
i)
$256 \quad 98.62 \%$ o
command: report statistics statistics report

\begin{tabular}{lrr} 
fault class & $\begin{array}{r}\text { \#faults } \\
\text { (coll.) }\end{array}$ & $\begin{array}{r}\text { \#faults } \\
\text { (total) }\end{array}$ \\
\hline FU (full) & 351 & 582 \\
\hline DS (det_simulation) & 321 & 546 \\
DI (det_impTication) & 20 & 26 \\
TI (tied) & 2 & 2 \\
AU (atpg_untestable) & 8 & 8 \\
\hline test_coverage & $-97.71 \%$ & $98.62 \%$ \\
fault_coverage & $97.15 \%$ & $98.28 \%$ \\
atpg_effectiveness & $100.00 \%$ & $100.00 \%$ \\
\hline \#test_patterns & & 46 \\
\#simuTated_patterns & & 288 \\
CPU_time (secs) & & 2.4 \\
\hline
\end{tabular}

ATPG) save patterns results/it_scan.patterns ATPG> exit. 
15 sunburn\% fastscan ilu -lib dft_stuff/genlib.atglib -nogui

SETUP> dofile dft_stuff/ilu. do

/) command: add clocks 0 clear test_clk

l/ command: add scan group g1 dft_stuff/ilu.g1

/) command: add scan chain c1 g1 scan_in scan_out

// command: add pin constraint test_mode c1

if command: set system mode ATPG

i) Flattening netlist...

If Flattening process completed, user_gates=73 sim_gates=1247 PIs=81 P0s=25.

// Begin circuit learning analyses.

(1) - Begin circuit learning analyses.

1) Equivalent gates $=48$ classes $=24$ CPU time $=0.07 \mathrm{sec}$.

1) Learned gate functions: \#MUXs=40

/) Learning completed, implications $=368$, tied_gates $=17$, CPU time=0.08 sec.

// Begin scan chain identification process, memory elements $=16$.

// Reading group test procedure file dft stuff/ilu.g1.

if Chain $=c 1$ successfully traced with scan_cells $=16$.

/) Begin scan clock rules checking.

/) 2 scan clock/set/reset lines have been identified.

(1) all scan clocks successfully passed off-state check.

1/ All scan clocks successfully passed capture ability check.

/f Capture clock is set to TEST_CLK.

(1) command: add faults -all

i) command: run

(1) Simulation performed for \#gates = 1247 \#faults = 3874

(1) system mode = ATPG pattern source = internal patterns

/) \#patterns test \#faults \#faults \# eff. \# test process

/) simulated coverage in list detected patterns patterns CPU time

/f begin random patterns: capture clock = /TEST_CLK, observe point = MASTER

if begin random patterns: capture clock = /CLEAR, observe point = MASTER

/) begin random patterns: capture clock = none, observe point = MASTER

if deterministic ATPG invoked with abort limit $=30$

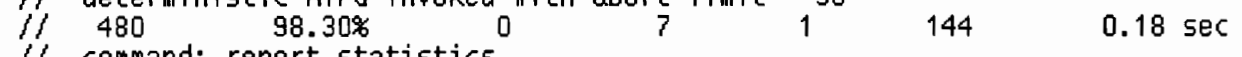

// command: report statistics statistics report

\begin{tabular}{lrr} 
fault class & $\begin{array}{r}\text { \#faults } \\
\text { (coll.) }\end{array}$ & $\begin{array}{r}\text { \#faults } \\
\text { (total) }\end{array}$ \\
\hline FU (ful1) & 2586 & 4126 \\
\hline DS (det_simulation) & 2371 & 3874 \\
DI (det_implication) & 132 & 166 \\
TI (tied) & 16 & 16 \\
AU (atpg_untestabie) & 67 & 70 \\
test_coverage & $-97.39 \%$ & $98.30 \%$ \\
fault_coverage & $96.79 \%$ & $97.92 \%$ \\
atpg_effectiveness & $100.00 \%$ & $100.00 \%$ \\
- \#test_patterns & & 144 \\
\#simuTated_patterns & & 480 \\
CPU_time (secs) & & 9.0 \\
\hline
\end{tabular}

ATPG > save patterns results/ilu.patterbs ATPG> exit

Figure 59: the ILU block log file 